\title{
QUANTUM VARIANCE OF MAASS-HECKE CUSP FORMS
}

\author{
DISSERTATION \\ Presented in Partial Fulfillment of the Requirements for \\ the Degree Doctor of Philosophy in the Graduate \\ School of the Ohio State University
}

By

Peng Zhao

$* * * * *$

The Ohio State University
2009

Dissertation Committee:

Approved by

Professor Wenzhi Luo, Advisor

Professor James Cogdell

Professor Manfred Einsiedler

Advisor

Graduate Program in Mathematics 



\begin{abstract}
In this thesis we study quantum variance for the modular surface $X=\Gamma \backslash \mathbb{H}$ where $\Gamma=S L_{2}(\mathbb{Z})$ is the full modular group. This is an important problem in mathematical physics and number theory concerning the mass equidistribution of Maass-Hecke cusp forms on the arithmetic hyperbolic surfaces. We evaluate asymptotically the quantum variance, which is introduced by S. Zelditch and describes the fluctuations of a quantum observable. We show that the quantum variance is equal to the classical variance of the geodesic flow on $S^{*} X$, the unit cotangent bundle of $X$, but twisted by the central value of the Maass-Hecke $L$-functions.

Our approach is via Poincare series and Kuznetsov trace formula, which transfer the spectral sum into the sum of Kloosterman sums. The treatment of the nondiagonal terms contributions is subtle and forms the core of this thesis. It turns out that the continuous spectrum part is not negligible and contributes to the main term, but in general, it is small in the cuspidal subspace. We then make use of Watson's explicit triple product formula to determine the leading term in the asymptotic formula for the quantum variance and analyze its structure, which leads to our main result.
\end{abstract}


Dedicated to my parents 


\section{ACKNOWLEDGMENTS}

I express my deepest thanks and gratitude to my advisor, Professor Wenzhi Luo, for his patience, guidance and constant encouragement which made this work possible. I am very grateful for his enlightening discussions and suggestions as well as the intellectual support he has provided.

I would like to thank the members of my dissertation committee, Professor James Cogdell and Professor Manfred Einsiedler for their valuable time and generous help.

I would like to acknowledge my special thanks to Professor Peter Sarnak and Professor Zeev Rudnick for their warmful encouragement and their interests to this work.

Last but not least, I wish to thank my parents, my wife and my daughter for their constant support. 


\section{VITA}

1978.7

1999.6

2002.6

2009.6
Born in Linyi, China

B.Sc. in Mathematics,

Shandong University, China

M.Sc. in Mathematics,

Shandong University, China

(expected) Ph.D. in Mathematics, The Ohio State University

\section{FIELDS OF STUDY}

Major Field: Mathematics

Specialization: Number Theory 


\section{TABLE OF CONTENTS}

Abstract ............................... ii

Dedication ................................... ii

Acknowledgments . . . . . . . . . . . . . . . . . . iv

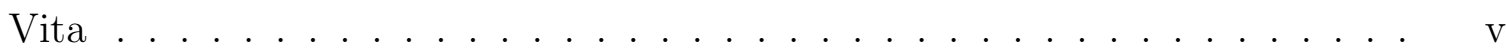

$\begin{array}{ll}\text { CHAPTER PAGE } & \text { PAT }\end{array}$

$1 \quad$ Introduction . . . . . . . . . . . . . . . . . . . . 1

2 Poincaré Series . . . . . . . . . . . . . . . . . 16

2.1 Preliminary . . . . . . . . . . . . . . . . 16

2.2 The Kuznetsov Formula . . . . . . . . . . . . . . . . . . . . . . 20

2.2.1 The Diagonal Term $(2.5) \ldots \ldots \ldots \ldots . \ldots . . \ldots 22$

2.2.2 The Non-diagonal Term $(2.7)$. . . . . . . . . . . 23

2.2.3 The Continuous Spectrum Part (2.6) . . . . . . . . . . 28

2.3 The Asymptotic Formula for the Variance . . . . . . . . . . . . . . 33

$3 \quad$ Symmetry Properties of $B \ldots \ldots \ldots$. . . . . . . . . . . . 37

3.1 Self-adjointness of Laplacian for $B \ldots \ldots$. . . . . . . . . . . 37

3.2 Self-adjointness of Hecke Operators for $B$. . . . . . . . . . . . 41

$4 \quad$ Extension and Diagonalization of $B \ldots \ldots \ldots$

$5 \quad$ Eigenvalues of $B \ldots \ldots \ldots \ldots \ldots \ldots$

5.1 Watson's Formula . . . . . . . . . . . . . . . 54

5.2 The Kuznetsov Formula . . . . . . . . . . . . . . . . . . . 55

5.2 .1 The Diagonal Term . . . . . . . . . . . . . . 57

5.2 .2 The Non-diagonal Term . . . . . . . . . . . . . . 58

5.2.3 The Continuous Spectrum Part . . . . . . . . . . . 60

5.3 Conclusion . . . . . . . . . . . . . . . . 63 
Bibliography 


\section{CHAPTER 1}

\section{INTRODUCTION}

Let $\Gamma=S L(2, \mathbb{Z})$ be the full modular group and $X=\Gamma \backslash \mathbb{H}$ be the corresponding modular surface, where $\mathbb{H}$ denotes the upper half plane. $\mathbb{H}$ is equipped with the Poincaré metric,

$$
d s^{2}=y^{-2}\left(d x^{2}+d y^{2}\right)
$$

with constant negative curvature -1 . On $X$ we have the normalized invariant hyperbolic measure

$$
d \mu=\frac{1}{\operatorname{Area}(X)} \frac{d x d y}{y^{2}},
$$

and the Beltrami-Laplace operator

$$
\Delta=y^{2}\left(\frac{\partial^{2}}{\partial x^{2}}+\frac{\partial^{2}}{\partial y^{2}}\right) .
$$

Define $\mathcal{A}(X)$ to be the space of automorphic functions, i.e.

$$
\mathcal{A}(X)=\{f: \mathbb{H} \rightarrow \mathbb{C} \mid f(\gamma z)=f(z), \quad \text { for all } \quad \gamma \in \Gamma\}
$$

and $\mathcal{A}_{s}(X)$ be the subspace of automorphic forms (i.e. Maass forms consistings of all $f \in \mathcal{A}(X)$ which have continuous second derivatives and satisfy

$$
(\Delta+s(1-s)) f=0 \quad \text { with } \quad s=\frac{1}{2}+i t .
$$

Let $\mathcal{L}(X)$ be the Hilbert space of square integrable automorphic functions with the inner product

$$
\langle f, g\rangle=\int_{X} f(z) \bar{g}(z) d \mu .
$$


Define $\mathcal{C}(X)$ to be the space of smooth, bounded automorphic functions whose zero-th terms vanish in their Fourier expansion at $\infty$. Automorphic forms in the space $\mathcal{C}(X)$ are called cusp forms.

Define $\mathcal{E}(X)$ to be the space of incomplete Eisenstein series

$$
E(z \mid \psi)=\sum_{\gamma \in \Gamma_{\infty} \backslash \Gamma} \psi(\operatorname{Im}(\gamma z))
$$

with $\psi$ any smooth compactly supported function on $\mathbb{R}^{+}$and

$$
\Gamma_{\infty}=\left\{\gamma=\left(\begin{array}{ll}
1 & * \\
0 & 1
\end{array}\right) \in \Gamma\right\}
$$

It is well known that the space $\mathcal{L}(X)$ has the following orthogonal decomposition $[17]$

$$
\mathcal{L}(X)=\tilde{\mathcal{C}}(X) \oplus \tilde{\mathcal{E}}(X),
$$

where the tilde means the closure in the Hilbert space $\mathcal{L}(X)$.

In addition to the Laplace operator, we have the commuting family of Hecke operators $T_{n}, n \geq 1$ acting on $\mathcal{A}(X)$ by

$$
T_{n} f(z)=\frac{1}{\sqrt{n}} \sum_{\gamma \in \Gamma \backslash G_{n}} f(\gamma z)
$$

where

$$
G_{n}=\left\{\left(\begin{array}{ll}
a & b \\
c & d
\end{array}\right) \in M_{2}(\mathbb{Z}), a d-b c=n\right\}
$$

and it is well known that all $T_{n}$ 's commute with $\Delta$. Let $\lambda_{f}(n)$ be the eigenvalue of the Hecke operator $T_{n}$ for an automorphic function $f$. The eigenvalues are multiplicative and satisfy

$$
\lambda_{f}(m) \lambda_{f}(n)=\sum_{d \mid(m, n)} \lambda_{f}\left(\frac{m n}{d^{2}}\right) .
$$


Recall that Hecke operators commute with each other and are self-adjoint, thus we can choose an orthonormal basis $\left\{\phi_{j}(z)\right\}$ for the space of cusp forms $\mathcal{C}(X)$, such that for any $n$,

$$
T_{n} \phi_{j}(z)=\lambda_{j}(n) \phi_{j}(z)
$$

Functions in $\mathcal{A}_{s}(X)$ which are simultaneous eigenfunctions for all $T_{n}$ are called MaassHecke eigenforms, i.e., the Maass form $\phi$ satisfies

$$
\Delta \phi+\lambda \phi=0, \quad T_{n} \phi=\lambda_{\phi}(n) \phi
$$

The Maass-Hecke eigenform $\phi$ with Laplacian eigenvalue $\frac{1}{4}+t^{2}$ has a Fourier expansion of the type

$$
\phi(z)=\sqrt{y} \sum_{n \neq 0} \rho_{\phi}(n) K_{i t}(2 \pi|n| y) e(n x)
$$

where $K_{i t}$ is the $K$-Bessel function and $\rho_{\phi}(n)$ is proportional to the $n$-th Hecke eigenvalue $\lambda_{\phi}(n)$, i.e.

$$
\rho_{\phi}(n)=\lambda_{\phi}(n) \rho_{\phi}(1) .
$$

We are mainly interested in the distribution of the probability measures on $X$ $d \mu_{j}:=\left|\phi_{j}(z)\right|^{2} d \mu$, as $j \rightarrow \infty$.

The quantum unique ergodicity conjecture (QUE) is a deep problem concerning the mass equidistribution of automorphic forms for $X$. The goal of QUE is to understand the limiting behavior of Laplacian eigenfunctions. This is an important problem in mathematical physics and number theory and it has origin from Quantum Chaos, which attempts to understand the relation between classical physics and quantum physics. In the number theory setting, we consider the case of Maass cusp forms associated with large Laplace eigenvalues for $X$.

Schnirelman [44], Colin de Verdiere [4] and Zelditch [51] have shown in general that if the geodesic flow on the unit cotangent bundle of a manifold $Y$ is ergodic, 
(by the similar definition for $X$, we form $d \mu_{j}$ and $d \mu$ on $Y$ ) there exist a full density subsequence $\left\{\phi_{j_{k}}\right\}$ of $\left\{\phi_{j}\right\}$ become equidistributed as $j_{k} \rightarrow \infty$, i.e.

$$
\lim _{j_{k} \rightarrow \infty} \int_{Y} \psi d \mu_{j_{k}}=\int_{Y} \psi d \mu
$$

for any Schwartz function $\psi \in C_{0}^{\infty}(Y)$. The geodesic flow being ergodic means almost all orbits of the flow become equidistributed. The above result can be viewed as the quantum analogue of the geodesic flow being geodesic. Works of Hejhal-Rackner [14] and Rudnick-Sarnak [40] suggest that there are no exceptional subsequences such that $d \mu_{j} \nrightarrow d \mu$ as $j \rightarrow \infty$, that is called quantum unique ergodicity.

More precisely, the quantum unique ergodicity conjecture for $X$ states that:

Conjecture (Rudnick and Sarnak). For any Jordan measurable compact region $A \subset$ $X$, we have

$$
\lim _{j \rightarrow \infty} \int_{A} d \mu_{j}=\int_{A} d \mu
$$

This is equivalent to

$$
\lim _{j \rightarrow \infty} \int_{X} \psi d \mu_{j}=\int_{X} \psi d \mu
$$

for any Schwartz function $\psi \in C_{0}^{\infty}(X)$.

We will mention several important approaches to this conjecture shortly.

Next we describe the quantization introduced by Zelditch [51].

We realize the upper half plane $\mathbb{H}$ as the homogeneous space $S L_{2}(\mathbb{R}) / S O_{2}(\mathbb{R})$ and $Y=S L_{2}(\mathbb{Z}) \backslash S L_{2}(\mathbb{R})$ as the unit cotangent bundle for $X$ and functions on $X$ can be viewed as $\mathrm{SO}_{2}(\mathbb{R})$ invariant functions on $Y$. The motion along geodesics on $X$ gives rise to a Hamiltonian flow $\mathcal{G}_{t}$ on $Y$

$$
\Gamma g \rightarrow \Gamma g\left(\begin{array}{cc}
e^{\frac{t}{2}} & 0 \\
0 & e^{-\frac{t}{2}}
\end{array}\right), \quad t \in \mathbf{R}
$$


Recall that the geodesic flow on a negative curvature manifold is ergodic [15], see [1] for $X$, we can consider this Hamiltonian flow as a classically chaotic system. From this system, we construct a quantum model as follows.

Let $C_{0}^{\infty}(Y)$ be the space of smooth functions on $Y$ which decay rapidly at cusps and similarly we define the space $C_{0}^{\infty}(X)$. Let $C_{0,0}^{\infty}(X)$ be the subspace of $C_{0}^{\infty}(X)$ consisting of smooth functions whose zeroth Fourier coefficient $\int_{0}^{1} \psi d x$ is zero for $y$ large enough and mean zero, i.e. $\int_{X} \psi d \mu=0$. We can view any function $\psi \in$ $C_{0}^{\infty}(Y)$ as a classical observable which includes information about the system. The quantization of Zelditch relates the classical observable $\psi$ to the quantum observable, a self-adjoint operator $O p(\psi)$ on the Hilbert space of square integrable automorphic forms $\mathcal{L}(X)$. To be precise,

$$
\left\langle O p(\psi) \phi_{j}, \phi_{j}\right\rangle=\int_{X} O p(\psi) d \mu_{j}
$$

gives the value of this quantum observable at state $\phi_{j}$. For $\psi \in C_{0}^{\infty}(X), O p(\psi)$ is the multiplication operator $(O p(\psi) h)(z)=\psi(z) h(z)[34]$ and therefore the inner product becomes $\left\langle O p(\psi) \phi_{j}, \phi_{j}\right\rangle=\int_{X} \psi d \mu_{j}$.

Returning to the above quantum unique ergodicity, there are several different approaches to this conjecture so far.

From Watson's explicit triple product formula [49], let $f$ be a Maass-Hecke eigenform,

$$
\left|\int_{X} f(z) d \mu_{j}\right|^{2}=\frac{\Lambda\left(\frac{1}{2}, f \otimes \operatorname{sym}^{2} \phi_{j}\right) \Lambda\left(\frac{1}{2}, f\right)}{\Lambda\left(1, \operatorname{sym}^{2} f\right) \Lambda\left(1, \operatorname{sym}^{2} \phi_{j}\right)^{2}},
$$

where $\Lambda$ is the completed $L$-function with the infinite part included. The special values $L\left(1, \mathrm{sym}^{2} \cdot\right)$ have not much effect since we have the following effective bounds due to Iwaniec and Hoffstein-Lockhart, for any $\epsilon>0$ :

$$
\lambda_{j}^{-\epsilon} \ll_{\epsilon} L\left(1, \operatorname{sym}^{2} \phi_{j}\right) \ll_{\epsilon} \lambda_{j}^{\epsilon} .
$$


Thus, the subconvexity bound of the degree 6 triple product $L$-function

$$
L\left(\frac{1}{2}, f \otimes \operatorname{sym}^{2} \varphi_{j}\right)=o\left(\lambda_{j}^{\frac{1}{2}}\right)
$$

would imply the above conjecture. Currently the best bound is

$$
L\left(\frac{1}{2}, f \otimes \operatorname{sym}^{2} \varphi_{j}\right)=O_{\epsilon, f}\left(\lambda_{j}^{\frac{1}{2}+\epsilon}\right)
$$

which follows from the Phragmen-Lindelof convexity principle. The General Riemann hypothesis would imply the Lindelof Hypothesis

$$
L\left(\frac{1}{2}, f \otimes \operatorname{sym}^{2} \varphi_{j}\right)=O_{\epsilon, f}\left(\lambda_{j}^{\epsilon}\right)
$$

hence QUE holds under GRH. Moreover, it predicts the rate of convergence for the equidistribution

$$
\int_{X} f(z) d \mu_{j}=O_{\epsilon, f}\left(\lambda_{j}^{-\frac{1}{4}+\epsilon}\right)
$$

In [29], Luo and Sarnak proved the quantum unique ergodicity with the above rate on the average, i.e.

Theorem 1.1. Let $f \in C_{0,0}^{\infty}(X)$, then

$$
\sum_{\lambda_{j} \leq \lambda}\left|\int_{X} f d \mu_{j}\right|^{2} \ll \lambda^{\frac{1}{2}+\epsilon}
$$

where the implied constant depends on $\epsilon$ and $f$.

For the continuous spectrum of $X$, they showed that, if $A, B$ are two compact Jordan measurable subsets of $X$, then

$$
\lim _{t \rightarrow \infty} \frac{\mu_{t}(A)}{\mu_{t}(B)}=\frac{\operatorname{Area}(A)}{\operatorname{Area}(B)},
$$

where

$$
\mu_{t}(A)=\int_{A}\left|E\left(z, \frac{1}{2}+i t\right)\right|^{2} \frac{d x d y}{y^{2}}
$$


Actually they proved that as $t \rightarrow \infty$,

$$
\mu_{t}(A) \sim \frac{48}{\pi} \operatorname{Area}(A) \log t
$$

Jakobson [20] has extended Theorem 1.1 to the microlocalizations of $\mu_{t}$ to $S^{*} X$, the unit cotangent bundle of $X$ (the Wigner distributions on $P S L_{2}(\mathbb{Z}) \backslash P S L_{2}(\mathbb{R})$ ).

For CM forms, forms with complex multiplication, QUE also holds by the work of Sarnak [43]. By the above Watson's triple-product formula, one can deduce the quantum unique ergodicity conjecture from the subconvexity bound for the triple product $L$-function at the central point. For CM forms, the advantage is the degree 6 triple product $L$-function factorizes into $L$-functions for which the subconvexity bound can be established (one is degree 4 Rankin-Selberg $L$-function and the other one $L(s, f)$ is degree 2$)$.

Lindenstrauss [26] has established quantum unique ergodicity for compact arithmetic quotients of $\mathbb{H}$ by ergodic methods.

Theorem 1.2. Let $M=\Gamma \backslash \mathbb{H}$ with $\Gamma$ a congruence lattice over $\mathbf{Q}$. Then for compact $M$, the only arithmetic quantum limit is the normalized invariant measure $d \mu$. For $M$ non-compact, any arithmetic quantum limit is of the form $c d \mu$ with $0 \leq c \leq 1$.

Therefore, in the case of $X=\Gamma \backslash \mathbb{H}$, quantum unique ergodicity conjecture is true up to a constant $c$, where $0 \leq c \leq 1$.

Holowinsky and Soundararajan recently proved the holomorphic version of QUE for $X[13]$.

Theorem 1.3. Let $f$ be a Hecke cusp eigenform of weight $k$.

(i) Let $\phi$ be a Hecke-Maass cusp form, then

$$
\left|\mu_{f}(\phi)\right| \ll_{\phi, \epsilon}(\log k)^{-\frac{1}{30}+\epsilon}
$$


(ii) Let $\psi$ be a fixed smooth function compactly supported in $(0, \infty)$. Then

$$
\left|\left(\mu_{f}(E(\cdot \mid \psi))-\frac{3}{\pi} \mu(E(\cdot \mid \psi))\right)\right| \ll_{\psi, \epsilon}(\log k)^{-\frac{2}{15}+\epsilon} .
$$

where

$$
\mu_{f}(\psi)=\int_{X} \psi(z) y^{k}|f(z)|^{2} \frac{d x d y}{y^{2}}
$$

Combined with the work of Lindenstrauss, Soundararajan [45] has proven the original version of the QUE conjecture, i.e., for noncompact arithmetic surfaces in Theorem 1.2, $c=1$.

Therefore, for QUE in the case of arithmetic surfaces, the case of holomorphic forms on compact arithmetic surfaces is still unsolved.

We will study this equidistribution problem by calculating the quantum variance. This variance sum

$$
S_{\psi}(\lambda)=\sum_{\lambda_{j} \leq \lambda}\left|\mu_{j}(\psi)\right|^{2}
$$

is first introduced by Zelditch [51]. It describes the fluctuations of a quantum observable $\left\langle O p(\psi) \mu_{j}, \mu_{j}\right\rangle=\mu_{j}(\psi)$ for $\psi \in C_{0,0}^{\infty}(X)$. For the classical observable $\psi \in C_{0,0}^{\infty}(Y)$, where $Y=\Gamma \backslash S L_{2}(R)$, as studied by Ratner [39], the fluctuations along a generic orbit of the geodesic flow obey the central limit theorem, i.e. the distribution of

$$
\frac{1}{\sqrt{T}} \int_{0}^{T} \psi\left(\mathcal{G}_{t}(g)\right) d t
$$

become Gaussian with mean 0 and the variance given by the non-negative Hermitian form on $C_{0,0}^{\infty}(Y)$ :

$$
V_{\mathrm{C}}(\phi, \psi)=\int_{-\infty}^{\infty} \int_{\Gamma \backslash S L(2, \mathbf{R})} \phi\left(g\left(\begin{array}{cc}
e^{\frac{t}{2}} & 0 \\
0 & e^{-\frac{t}{2}}
\end{array}\right)\right) \overline{\psi(g)} d g d t .
$$

Restricting $V_{C}$ to $C_{0,0}^{\infty}(X)$, it can be diagonalized by the Maass cusp forms $\phi$ with Laplacian eigenvalue $\lambda=\frac{1}{4}+t^{2}$ and the eigenvalue of $V_{C}$ on $\phi$ equals

$$
V_{\mathrm{C}}(\phi, \phi)=\frac{\left|\Gamma\left(\frac{1}{4}-\frac{i t}{2}\right)\right|^{4}}{2 \pi\left|\Gamma\left(\frac{1}{2}-i t\right)\right|^{2}}
$$


It is shown that the means of the classical observable and the quantum observable are the same by the work of Zelditch [51]. There should be a deep relation between the quantum variance and the classical variance. In [9] and [10], the physicists conjectured that for generic quantum chaotic system, one expects a central limit theorem for the statistical fluctuations of the quantum observable. For the quantum variance, it should correspond to the classical variance $V_{C}(\psi, \psi)$.

In [34], Luo and Sarnak examined the analogous quantum variance for holomorhic Hecke eigenforms. The automorphic forms they considered are the holomorphic cusp forms in $S_{k}(\Gamma)$ of even integral weight $k$ for $\Gamma . S_{k}(\Gamma)$ has a Hilbert space structure with respect with the Petersson inner product. Let $H_{k}$ be the orthonormal basis of Hecke eigenforms for $S_{k}(\Gamma)$. For $\psi \in C_{0,0}^{\infty}(X)$, they studied the variance of $\mu_{f}(\psi)$ as $f$ runs through $H_{k}$. They considered a weighted version of the quantum variance sum by inserting arithmetic weights $L\left(1, \operatorname{Sym}^{2} f\right)$ associated with the symmetric-square $L$-function (these appear naturally in the Petersson formula), they proved that for $\phi, \psi \in C_{0,0}^{\infty}(X)$, as $K \rightarrow \infty$,

$$
\sum_{k \leq K} \sum_{f \in H_{k}} L\left(1, \operatorname{Sym}^{2} f\right) \mu_{f}(\phi) \overline{\mu_{f}(\psi)} \sim B(\phi, \psi) K
$$

where $B(\phi, \psi)$ is a non-negative Hermitian form on $C_{0,0}^{\infty}(X)$. It turns out that $B(\phi, \psi)$ has some important symmetric properties. Thus $B$ is diagonalized by the orthonormal basis of Maass-Hecke cusp forms and the eigenvalues of $B$ at $\psi_{j}$ is $\frac{\pi}{2} L\left(\frac{1}{2}, \psi_{j}\right)$. We will turn to these similar properties for our case in Chapter 3.

In this thesis, we generalize the result in [34] to Maass cusp forms, i.e., we consider the quantum variance of Masse-Hecke cusp forms. In contrast to the case of the holomorphic cusp forms, the real analytic case is more challenging and complicated. New difficulties arise when one applies the Kuznetsov formula instead of the Petersson formula, in view of the occurrence of the continuous spectrum contribution, the subtle analysis of the hypergeometric functions and the sum of Kloosterman sum weighted 
by more involved Hankel transform. It turns out that the quantum variance is equal to the classical variance of the geodesic flow on $S^{*} X$, the unit cotangent bundle of $X$, but twisted by the central value of the Maass-Hecke $L$-functions. More precisely, we obtain the following:

Theorem 1.4. Let $\varphi_{j}(z)$ be the $j$-th Maass-Hecke eigenform, with the Laplacian eigenvalues $\lambda_{j}=\frac{1}{4}+t_{j}^{2}$. For $\phi, \psi \in \mathcal{C}(X)$, We have

$$
\sum_{\lambda_{j} \leq \lambda} \mu_{j}(\phi) \overline{\mu_{j}(\psi)} \sim \lambda^{\frac{1}{2}} V(\phi, \psi)
$$

$V$ is diagonalized by the orthonormal basis $\left\{\phi_{j}\right\}$ of Maass-Hecke cusp forms and the eigenvalue of $V$ at a Maass-Hecke cusp form $\phi$ is

$$
L\left(\frac{1}{2}, \phi\right) V_{\mathrm{C}}(\phi, \phi)
$$

In fact, we prove a weighted version of (1.1). The weights are mildly varying and have no effect on this asymptotic. To prove this theorem, we first establish a preliminary theorem for the incomplete Poincare series with the test functions satisfying some analytic conditions in view of the Kuznetsov formula. We then remove the analyticity condition on the test functions by using Fourier transform to obtain the general asymptotic formula for the quantum variance sum. For the incomplete Poincaré series, we can take the advantage of unfolding the integral and represent the integral as expressions involving the Fourier coefficients in quadratic polynomials which allows us to apply the Kuznetsov formula and transfer the spectral sum into the sum of Kloosterman sums. The treatment of the non-diagonal terms contributions is subtle and forms the core of my thesis. It turns out that the continuous spectrum part is not negligible and contributes to the main term. The preliminary theorem is as follows. 
Let $h(t)$ be a smooth function with compact support. Define the Poincaré series $(m \neq 0):$

$$
P_{h, m}(z)=\sum_{\gamma \in \Gamma_{\infty} \backslash \Gamma} h(y(\gamma z)) e(m x(\gamma z))
$$

For the continuous spectrum part, we consider the Eisenstein series $E(z, s)$, and define

$$
\mu_{t}(\psi)=\int_{X} \psi(z)\left|E\left(z, \frac{1}{2}+i t\right)\right|^{2} \frac{d x d y}{y^{2}} .
$$

Theorem 1.5. Let $\varphi_{j}$ denote the eigenfunctions of Laplacian, with the corresponding eigenvalues $\lambda_{j}=\frac{1}{4}+t_{j}^{2}$. For $m_{1}, m_{2} \neq 0, h_{1}, h_{2}$ be smooth functions with compact support for $i=1,2$, fix $\epsilon>0$, we have

$$
\begin{aligned}
& \sum_{t_{j}}\left(e^{-\left(\frac{t_{j}-T}{T^{1-\epsilon}}\right)^{2}}+e^{-\left(\frac{t_{j}+T}{T^{1-\epsilon}}\right)^{2}}\right) L\left(1, \operatorname{sym}^{2} \varphi_{j}\right) \mu_{j}\left(P_{h_{1}, m_{1}}\right) \overline{\mu_{j}}\left(P_{h_{2}, m_{2}}\right) \\
+ & \int_{0}^{\infty}\left(e^{-\left(\frac{t-T}{T^{1-\epsilon}}\right)^{2}}+e^{-\left(\frac{t+T}{T^{1-\epsilon}}\right)^{2}}\right)|\zeta(1+2 i t)|^{2} \mu_{t}\left(P_{h_{1}, m_{1}}\right) \overline{\mu_{t}}\left(P_{h_{2}, m_{2}}\right) d t \\
= & T^{1-\epsilon} B\left(P_{h_{1}, m_{1}}, P_{h_{2}, m_{2}}\right)+O\left(T^{\frac{1}{2}-\epsilon}\right),
\end{aligned}
$$

The Laplacian $\Delta$ and the Hecke operators $T_{n}$ are self-adjoint with respect to $B$.

Remark 1.1. (i) The weight $e^{-\left(\frac{t_{j}-T}{T^{1-\epsilon}}\right)^{2}}+e^{-\left(\frac{t_{j}+T}{T^{1-\epsilon}}\right)^{2}}$ is in effect localizing the spectrum sum to the range

$$
\left|t_{j}-T\right| \ll T^{1-\epsilon}
$$

(ii) To prove (1.2), we apply the Kuznetsov trace formula, and analyze the contributions of the diagonal, non-diagonal and the continuous spectrum parts. The integral term in Theorem 1.5 comes from the continuous spectrum. It turns out the continuous spectrum part is not negligible and contributes to the main term. However, if we restrict to $L_{c u s p}(X)$, the continuous spectrum part is small in view of the following result [L-S1],

$$
\left|\mu_{t}(\phi)\right| \ll_{\phi, \epsilon}|t|^{-\frac{1}{6}+\epsilon}
$$


for a Maass cusp form $\phi$.

(iii) To determine $B(\phi, \psi)$ in Theorem 1.4 explicitly, we apply Watson's formula, converting the problem to study the triple product $L$-values, by means of approximate functional equation and Kuznetsov formula. Here we appeal to Jutila's subconvexity bound [23]

$$
L\left(\frac{1}{2}+i t, \psi_{j}\right) \ll\left(t_{j}+t\right)^{1 / 3+\epsilon} .
$$

(iv) We call the eigenvalue of $V$ at $\phi$ as the arithmatic variance or quantum variance of the Maass-Hecke cusp form $\phi$. It is consistent with the Feingold-Peres [10] prediction for the variance of generic chaotic systems, i.e., it recovers the classical variance of $\phi$

$$
\frac{\left|\Gamma\left(\frac{1}{4}-\frac{i t_{\phi}}{2}\right)\right|^{4}}{2 \pi\left|\Gamma\left(\frac{1}{2}-i t_{\phi}\right)\right|^{2}}
$$

given by Ratner [38] multiplied by the central value $L\left(\frac{1}{2}, \phi\right)$. As a by-product, we obtain that, for Maass-Hecke form $\psi, L\left(1 / 2, \psi \otimes \operatorname{sym}^{2}\left(\phi_{j}\right)\right)$ is non-vanishing for some $\phi_{j}$ and the central value $L\left(\frac{1}{2}, \psi\right)$ is non-negative.

(v) In order to apply the Kuznetsov trace formula, we consider the weighted version sums of the quantum variance in (1.2). The weights $L\left(1, \operatorname{sym}^{2} \varphi_{j}\right)$ are mildly varying. For them, we have the following bounds due to Iwaniec and HoffsteinLockhart:

$$
t_{j}^{-\epsilon} \ll_{\epsilon} L\left(1, \operatorname{sym}^{2} \varphi_{j}\right) \ll_{\epsilon} t_{j}^{\epsilon}
$$

Thus, we can remove these weights using the methods in [19], see also [18].

(vi) If we consider the cotangent bundle $S^{*} X=\Gamma \backslash G$ to $X$ and lift the measures $d \mu_{j}$ on $X$ to the Wigner distribution $d \omega_{j}$ on $S^{*} X$, we could extend our result to $S^{*} X$, but we do not pursue them in this thesis.

(vii) The quantum variance $V(\psi)$ is the same as the variance for the fluctuations of the arithmetic measures on the modular surface obtained by collecting all closed geodesics. See the work of Luo, Rudnick and Sarnak [28]. 
(viii) In [24], Kurlberg and Rudnick formulated an interesting analogous variance for the quantum cat map, a linear hyperbolic map of the torus $\mathbb{R}^{2} / \mathbb{Z}^{2}$.

We end the introduction with an outline of this thesis.

In Chapter 2, we first establish a preliminary version of Theorem 1.5 for the incomplete Poincare series $P_{m, h}$ with $h$ satisfing some special analytic conditions in order to apply the Kutznesov formula. Now these Poincare series form a dense subspace of $C_{0,0}^{\infty}(X)$ and unfolding method allows us to analyze $\mu_{j}\left(P_{m, h}\right)$ in terms of sums over Fourier coefficients. We average over the orthonormal basis for the cuspidal subspace by Kutznesov formula, and deal with the diagonal, non-diagonal terms separately. Here the subtle test functions arise due to the complicated hypergeometric functions. For non-diagonal terms, we apply the Fourier transform in (Bateman, volume 1, p. 59)

$$
\left(\frac{J_{2 i t}(x) \widehat{J}_{-2 i t}(x)}{\sinh (\pi t)}\right)(y)=-i \cos (x \cosh (\pi y)) .
$$

The continuous spectrum parts is not negligible and contributes to the main term. We then remove the analyticity condition on $h$ by using the Fourier transform to obtain the general asymptotic formula for the quantum variance sum $S_{\psi}(\lambda)$.

Chapter 3 and Chapter 4 are devoted to analyze the structure of the quardratic form $B$. In Chapter 3, we obtain the self-adjointness of $B$ with respect to the Laplacian $\Delta$ and the Hecke operators using the series expression as obtained in the Theorem 1.4. To be precise, we analyze the bilinear form $B$ and using the series expression as obtained in Chapter 2, we prove it is self-adjoint with respect to the Laplacian $\Delta$ and the Hecke operators $T_{n}, n \geq 1$, i.e., for Poincaré series $\psi_{1}, \psi_{2}, B$ satisfies the symmetries

$$
B\left(\Delta \psi_{1}, \psi_{2}\right)=B\left(\psi_{1}, \Delta \psi_{2}\right)
$$


and for $n \geq 1$,

$$
B\left(T_{n} \psi_{1}, \psi_{2}\right)=B\left(\psi_{1}, T_{n} \psi_{2}\right)
$$

This part is more complicated than the holomorphic case in [34] due to the subtle series expression of $B$.

In Chapter 4 , we then diagonalize and extend $B$ to $C_{0,0}^{\infty}(X)$. We restrict $B$ to the Maass-Hecke cusp forms. The continuous spectrum integral is small enough so we can obtain the first part of main Theorem 1.4. If we restrict $B$ to the MaassHecke cusp forms, it can be shown that $B$ is diagonalized by the orthonormal basis of Hecke-Maass cusp forms from the symmetry properties of $B$ obtained in Chapter 3 . The idea of the extension is choosing a partition of unity subordinate to a covering of $X$, then any $\psi \in C_{0,0}^{\infty}(X)$ can be written as a linear combination of $P_{h, m}$, where $h$ is smooth with compact support functions.

In Chapter 5, we establish the second part of Theorem 1.4. We compute the value of $B$ on $\phi(z)$, an even Maass-Hecke cuspidal eigenform. After applying Watson's formula, the quantum variance sum over $\phi_{j}$ boils down to averaging $L\left(1 / 2, \phi \otimes \operatorname{sym}^{2}\left(\phi_{j}\right)\right)$ with respect to the spectrum. By Rankin-Selberg theory, we can express this sum in a suitable series to which the Kuznetsov formula is applied. The non-diagonal terms has no contribution to the main term as in the holomorphic case. For the continuous spectrum, it can be shown that it is small enough by the Jutila's subconvexity bound [23]. The resulting asymptotics is used to determine explicitly the quantum variance. Therefore, we complete the proof of Theorem 1.4.

We sketch the logic graph of this thesis as follows. 


\section{The Logic Graph}

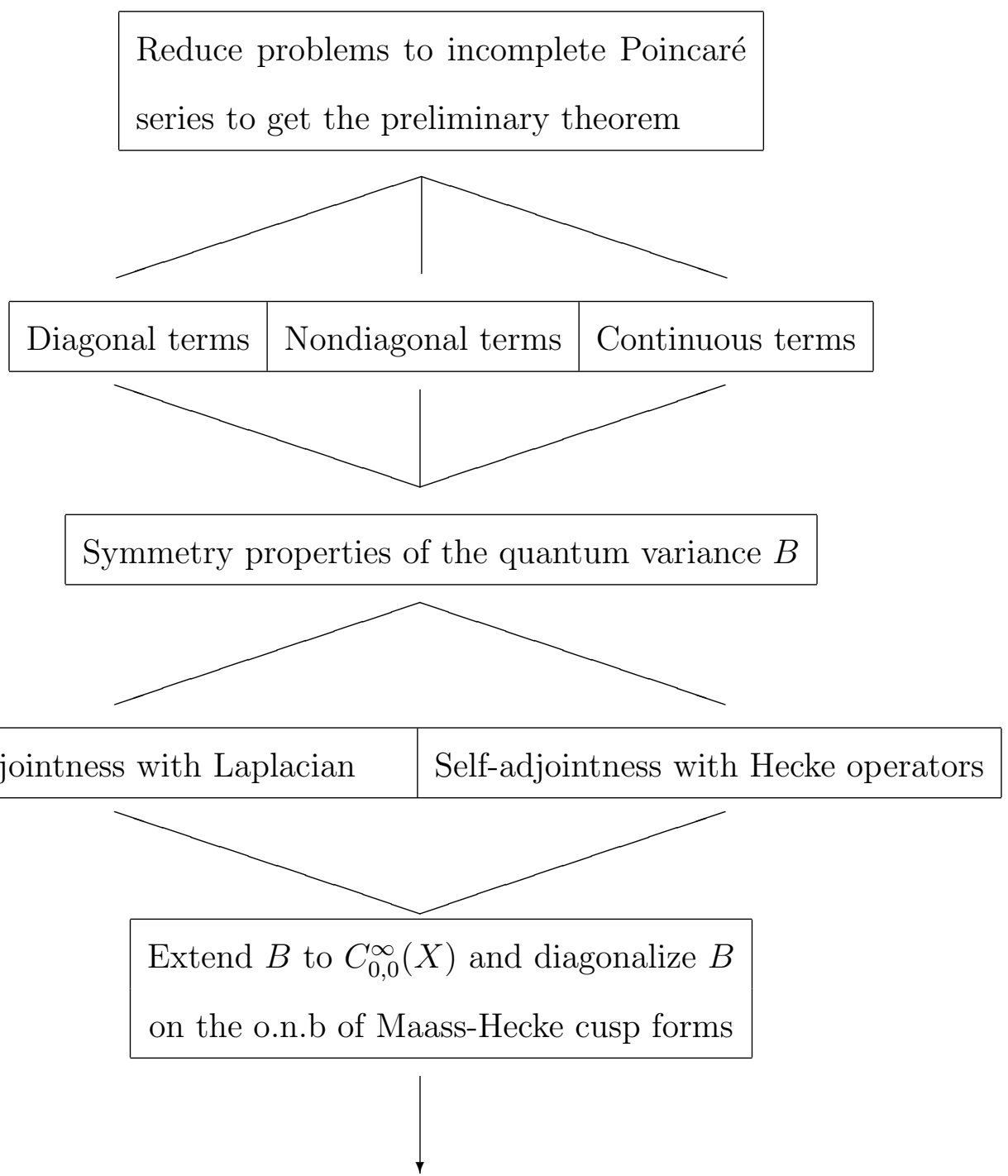

The eigenvalue of $B$ on a Maass-Hecke eigenform

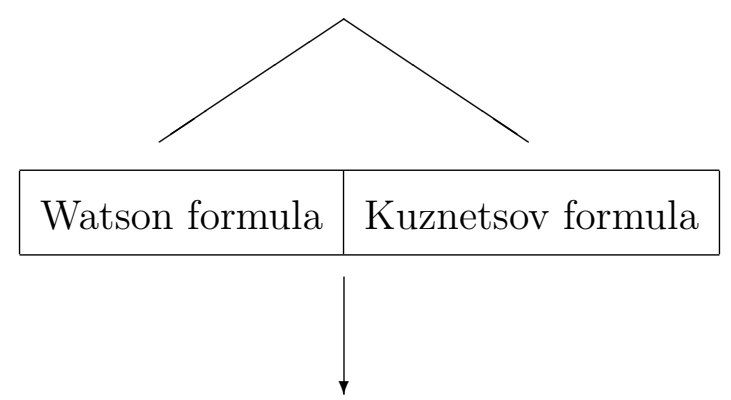

Quantum variance equals classical variance twisted by the central $L$-values 


\section{CHAPTER 2 POINCARÉ SERIES}

In this chapter we state the basic facts and prove auxiliary results that are needed for the main Theorem 1.3. We first establish a preliminary version of part (A) of Theorem 1.3 for Poincaré series $P_{m, h}$ with $h$ satisfying some special analytic conditions in order to apply the Kutznesov formula. Now Poincaré series form a dense subspace of $C_{0,0}^{\infty}(X)$ and the unfolding method allows us to analyze $\mu_{j}\left(P_{m, h}\right)$ in terms of sums over Fourier coefficients. We average over the orthonormal basis for the cuspidal subspace by the Kutznesov formula, and deal with the diagonal, non-diagonal terms separately, see details in Section 2.2. It turns out the continuous spectrum parts is not negligible and contributes to the main term. We then remove analyticity condition on $h$ by using Fourier transform to obtain the general asymptotic formula for the quantum variance sum $S_{\psi}(\lambda)$ in Section 2.3.

\subsection{Preliminary}

Let $\varphi_{j}$ denote the $j$-th Hecke-Maass eigenform with the corresponding Laplacian eigenvalue $\lambda_{j}=\frac{1}{4}+t_{j}^{2}$, Hecke eigenvalues $\lambda_{j}(n)$ and we normalize $\left\|\varphi_{j}\right\|_{2}=1$. Let $s_{j}=\frac{1}{2}+i t_{j}$, then $\varphi_{j}$ has the Fourier expansion

$$
\varphi_{j}(z)=\sqrt{y} \sum_{n \neq 0} \rho_{\phi}(n) K_{i t_{j}}(2 \pi|n| y) e(n x)
$$

where $K_{s}(y)$ is the $K$-Bessel function. 
Let $h(t)$ be an even real analytic function on $\mathbb{R}$ satisfying $h^{(n)}(t) \ll(1+|t|)^{-N}$ for any $n>0$ and any large $N$, and $h(t) \ll t^{10}$ when $t \rightarrow 0$. Define the Poincaré series $(m \in \mathbb{Z}, m \neq 0):$

$$
P_{h, m}(z)=\sum_{\gamma \in \Gamma_{\infty} \backslash \Gamma} h(y(\gamma z)) e(m x(\gamma z))
$$

Define the probability measures $d \mu_{j}(z)=\left|\varphi_{j}(z)\right|^{2} d \mu(z)$. We normalize

$$
\rho_{j}(n)=\cosh \left(\pi t_{j}\right)^{1 / 2} a_{j}(n),
$$

we have [31],

$$
\left|a_{j}(1)\right|^{2}=\frac{2}{L\left(1, \operatorname{sym}^{2} \varphi_{j}\right)}, \quad a_{j}(n)=a_{j}(1) \lambda_{j}(n)
$$

From the well-known facts

$$
\Gamma\left(\frac{1}{2}+i t_{j}\right) \Gamma\left(\frac{1}{2}-i t_{j}\right)=\frac{\pi}{\cosh \left(\pi t_{j}\right)},
$$

we have

$$
\begin{aligned}
<P_{h, m},\left|\varphi_{j}\right|^{2}>= & \int_{\Gamma \backslash \mathcal{H}}\left|\varphi_{j}(z)\right|^{2}\left(\sum_{\gamma \in \Gamma_{\infty} \backslash \Gamma} h(y(\gamma z)) e(m x(\gamma z))\right) d \mu(z) \\
= & \sum_{k \neq 0,-m} \rho_{j}(k) \rho_{j}(k+m) \int_{0}^{\infty} K_{i t_{j}}(r) K_{i t_{j}}\left(\left|1+\frac{m}{k}\right| r\right) h\left(\frac{r}{2 \pi|k|}\right) \frac{d r}{r} \\
= & \frac{2 \cosh \left(\pi t_{j}\right)}{L\left(1, \operatorname{sym}^{2} \varphi_{j}\right)} \sum_{k \neq 0,-m} \lambda_{j}(k) \lambda_{j}(k+m) \int_{(\sigma)} \frac{1}{2 \pi i} \frac{G(s)}{(\pi k)^{s}} 2^{-3} \\
= & \frac{\Gamma\left(\frac{s}{2}+i t_{j}\right) \Gamma\left(\frac{s}{2}-i t_{j}\right) f\left(k, t_{j}, s\right) d s}{L\left(1, \operatorname{sym}^{2} \varphi_{j}\right)} \sum_{k \neq 0,-m} \lambda_{j}(k) \lambda_{j}(k+m) \int_{(\sigma)} \frac{1}{2 \pi i} \frac{G(s)}{(\pi k)^{s}} 2^{-3} d s \\
& \frac{\Gamma\left(\frac{s}{2}+i t_{j}\right) \Gamma\left(\frac{s}{2}-i t_{j}\right)}{\Gamma\left(\frac{1}{2}+i t_{j}\right) \Gamma\left(\frac{1}{2}-i t_{j}\right)} f\left(k, t_{j}, s\right)
\end{aligned}
$$

where

$$
f\left(k, t_{j}, s\right)=(1+m / k)^{i t_{j}} \int_{0}^{1} \tau^{s / 2-1}(1-\tau)^{s / 2-1}\left(1+\frac{2 \tau m}{k}+\tau(m / k)^{2}\right)^{-s / 2-i t_{j}} d \tau
$$


Here we also applied the formulas (6.576) and (9.111) in [11]

$$
\begin{aligned}
& \int_{0}^{\infty} r^{s-1} K_{i t_{j}}(r) K_{i t_{j}}\left(\left(1+\frac{m}{k}\right) r\right) d r \\
= & 2^{-3-s}(1+m / k)^{i t_{j}} \frac{\Gamma\left(\frac{s}{2}\right)^{2}}{\Gamma(s)} \Gamma\left(\frac{s}{2}+i t_{j}\right) \Gamma\left(\frac{s}{2}-i t_{j}\right) F\left(\frac{s}{2}+i t_{j}, \frac{s}{2}, s, 1-\left(1+\frac{m}{k}\right)^{2}\right) \\
= & 2^{-3-s}(1+m / k)^{i t_{j}} \Gamma\left(\frac{s}{2}+i t_{j}\right) \Gamma\left(\frac{s}{2}-i t_{j}\right) \int_{0}^{1} \tau^{s / 2-1}(1-\tau)^{s / 2-1} \\
& \left(1+\frac{2 \tau m}{k}+\tau(m / k)^{2}\right)^{-s / 2-i t_{j}} d \tau .
\end{aligned}
$$

We can show $f\left(k, t_{j}, s\right)$ is even in $t_{j}$ by the integral identities of p.959 9.131 in [11] since

$$
\begin{aligned}
& \left(1+\frac{m}{k}\right)^{i t_{j}} F\left(\frac{s}{2}+i t_{j}, \frac{s}{2}, s, 1-\left(1+\frac{m}{k}\right)^{2}\right) \\
= & \left(1+\frac{m}{k}\right)^{i t_{j}} \frac{\Gamma(s) \Gamma\left(-i t_{j}\right)}{\Gamma\left(\frac{s}{2}-i t_{j}\right) \Gamma\left(\frac{s}{2}\right)} F\left(\frac{s}{2}+i t_{j}, \frac{s}{2}, i t_{j}+1,\left(1+\frac{m}{k}\right)^{2}\right) \\
+ & \left(1+\frac{m}{k}\right)^{-i t_{j}} \frac{\Gamma(s) \Gamma\left(i t_{j}\right)}{\Gamma\left(\frac{s}{2}+i t_{j}\right) \Gamma\left(\frac{s}{2}\right)} F\left(\frac{s}{2}-i t_{j}, \frac{s}{2},-i t_{j}+1,\left(1+\frac{m}{k}\right)^{2}\right)
\end{aligned}
$$

We also applied unfolding integral, Mellin transform:

$$
G(s)=\int_{0}^{\infty} h(y) y^{-s} \frac{d y}{y}
$$

For the Gamma functions in (2.1) we can get an asymptotic formula so that we can use the Mellin inversion:

$$
h(y)=\frac{1}{2 \pi i} \int_{\sigma-i \infty}^{\sigma+i \infty} G(s) y^{s} d s
$$

for any $\sigma>0$.

By Stirling Formula, for any vertical strip $0<a \leq \operatorname{Re}(s) \leq b$, as $t_{j} \rightarrow \infty$, we have

$$
\begin{aligned}
& \frac{\Gamma\left(\frac{s}{2}+i t_{j}\right)}{\Gamma\left(\frac{1}{2}+i t_{j}\right)} \sim \frac{\left(\frac{s}{2}+i t_{j}\right)^{\frac{s}{2}+i t_{j}-\frac{1}{2}} e^{-\frac{s}{2}}}{\left(\frac{1}{2}+i t_{j}\right) i t_{j} e^{-\frac{1}{2}}} \\
& =e^{\frac{1-s}{2}+\left(\frac{s}{2}+i t_{j}-\frac{1}{2}\right) \log \left(\frac{s}{2}+i t_{j}\right)-i t_{j} \log \left(\frac{1}{2}+i t_{j}\right)} \\
& =e^{\left(\frac{s}{2}-\frac{1}{2}\right) \log \left(\frac{1}{2}+i t_{j}\right)+\left(\frac{s}{2}+i t_{j}-\frac{1}{2}\right) \log \frac{\frac{s}{2}+i t_{j}}{\frac{1}{2}+i t_{j}}+\frac{1-s}{2}}
\end{aligned}
$$


Next we show

$$
e^{\left(\frac{s}{2}+i t_{j}-\frac{1}{2}\right) \log \frac{\frac{s}{2}+i t_{j}}{\frac{1}{2}+i t_{j}}+\frac{1-s}{2}}=1+O_{s}\left(t_{j}^{-1}\right)
$$

i. e.

$$
\left(\frac{s}{2}+i t_{j}-\frac{1}{2}\right) \log \frac{\frac{s}{2}+i t_{j}}{\frac{1}{2}+i t_{j}}+\frac{1-s}{2}=O_{s}\left(t_{j}^{-1}\right) .
$$

We have

$$
\begin{aligned}
& \left(\frac{s}{2}+i t_{j}-\frac{1}{2}\right) \log \frac{\frac{s}{2}+i t_{j}}{\frac{1}{2}+i t_{j}}+\frac{1-s}{2} \\
= & \left(\frac{s}{2}+i t_{j}-\frac{1}{2}\right)\left(\frac{\frac{s-1}{2}}{\frac{1}{2}+i t_{j}}+O_{s}\left(t_{j}^{-2}\right)\right)+\frac{1-s}{2} \\
= & \frac{s-1}{2}\left(\frac{\frac{s}{2}+i t_{j}-\frac{1}{2}}{\frac{1}{2}+i t_{j}}\right)+O_{s}\left(t_{j}^{-1}\right)+\frac{1-s}{2} \\
= & \frac{s-1}{2}\left(1+\frac{\frac{s}{2}-1}{\frac{1}{2}+i t_{j}}\right)+O_{s}\left(t_{j}^{-1}\right)+\frac{1-s}{2} \\
= & O_{s}\left(t_{j}^{-1}\right) .
\end{aligned}
$$

From (2.2) and (2.3), we obtain

$$
\frac{\Gamma\left(\frac{s}{2}+i t_{j}\right)}{\Gamma\left(\frac{1}{2}+i t_{j}\right)}=\left(\frac{1}{2}+i t_{j}\right)^{\frac{s-1}{2}}\left(1+O_{s}\left(t_{j}^{-1}\right)\right) .
$$

Similarly, we have

$$
\frac{\Gamma\left(\frac{s}{2}-i t_{j}\right)}{\Gamma\left(\frac{1}{2}-i t_{j}\right)}=\left(\frac{1}{2}-i t_{j}\right)^{\frac{s-1}{2}}\left(1+O_{s}\left(t_{j}^{-1}\right)\right) .
$$

Thus,

$$
\begin{aligned}
& \frac{\Gamma\left(\frac{s}{2}+i t_{j}\right) \Gamma\left(\frac{s}{2}-i t_{j}\right)}{\Gamma\left(\frac{1}{2}+i t_{j}\right) \Gamma\left(\frac{1}{2}-i t_{j}\right)} \\
= & \left(\frac{1}{4}+t_{j}^{2}\right)^{\frac{s-1}{2}}\left(1+O_{s}\left(t_{j}^{-1}\right)\right)=\left(t_{j}\right)^{s-1}\left(1+O_{s}\left(t_{j}^{-1}\right)\right) .
\end{aligned}
$$

as $t_{j} \rightarrow \infty$. 


\subsection{The Kuznetsov Formula}

The strategy to obtain the asymptotic for the variance

$$
S_{\psi}(\lambda)=\sum_{\lambda_{j} \leq \lambda}\left|\mu_{j}(\psi)\right|^{2}
$$

is to take a smoothed version of the above sum and apply the Kuznetsov trace formula.

From (2.1), (2.4) and Mellin inversion, we obtain

$$
<P_{h, m},\left|\mu_{j}\right|^{2}>=\frac{\pi}{4 t_{j} L\left(1, \operatorname{sym}^{2} \varphi_{j}\right)} \sum_{k \neq 0,-m} \lambda_{j}(k) \lambda_{j}(k+m) H\left(t_{j}, k, m\right)+O\left(t_{j}^{-1-\epsilon}\right),
$$

where

$$
H\left(t_{j}, k, m\right)=\int_{0}^{1}\left(\frac{1+\frac{m}{k}}{1+\frac{2 \tau m}{k}+\frac{\tau m^{2}}{k^{2}}}\right)^{i t_{j}} \frac{1}{\tau(1-\tau)} h\left(\frac{t_{j} \sqrt{\tau(1-\tau)}}{\pi k \sqrt{1+\frac{2 \tau m}{k}+\frac{\tau m^{2}}{k^{2}}}}\right) d \tau
$$

Note: In the holomorphic case (for weight $k$ modular form $f$ ) [34], we have $<P_{h, m}, \mu_{f}>=\frac{2 \pi^{2}}{(k-1) L\left(1, \operatorname{sym}^{2}(f)\right)} \sum_{r \geq 1} \lambda_{f}(r) \lambda_{f}(r+m) h\left(\frac{k-1}{4 \pi(r+m / 2)}\right)+O\left(k^{-1-\epsilon}\right)$

Thus, by the multiplicativity of Hecke eigenvalues, we have

$$
\begin{aligned}
& <P_{h_{1}, m_{1}},\left|\mu_{j}\right|^{2}>\overline{<P_{h_{2}, m_{2}},\left|\mu_{j}\right|^{2}>} \\
= & \frac{\pi^{2}}{16 t_{j}^{2} L^{2}\left(1, \mathrm{sym}^{2} \varphi_{j}\right)} \sum_{d_{1}\left|m_{1}, d_{2}\right| m_{2}} \sum_{k_{1}, k_{2}} \lambda_{j}\left(k_{1}\left(k_{1}+\frac{m_{1}}{d_{1}}\right)\right) \lambda_{j}\left(k_{2}\left(k_{2}+\frac{m_{2}}{d_{2}}\right)\right) \\
& H_{1}\left(t_{j}, d_{1} k_{1}, m_{1}\right) \overline{H_{2}\left(t_{j}, d_{2} k_{2}, m_{2}\right)}+O\left(t_{j}^{-2-\epsilon}\right) \\
= & \frac{\pi^{2}}{32 t_{j}^{2} L\left(1, \mathrm{sym}^{2} \varphi_{j}\right)} \sum_{d_{1}\left|m_{1}, d_{2}\right| m_{2} k_{1}, k_{2}} \sum_{j}\left(k_{1}\left(k_{1}+\frac{m_{1}}{d_{1}}\right)\right) \overline{a_{j}\left(k_{2}\left(k_{2}+\frac{m_{2}}{d_{2}}\right)\right)} \\
& H_{1}\left(t_{j}, d_{1} k_{1}, m_{1}\right) \overline{H_{2}\left(t_{j}, d_{2} k_{2}, m_{2}\right)}+O\left(t_{j}^{-2-\epsilon}\right) .
\end{aligned}
$$

Consider

$$
\begin{aligned}
& \sum_{j \geq 1}\left(e^{-\left(\left(t_{j}-T\right) / T^{1-\varepsilon}\right)^{2}}+e^{-\left(\left(t_{j}+T\right) / T^{1-\varepsilon}\right)^{2}}\right) L\left(1, \operatorname{sym}^{2} \varphi_{j}\right) \\
& <P_{h_{1}, m_{1}},\left|\mu_{j}\right|^{2}>\overline{<P_{h_{2}, m_{2}},\left|\mu_{j}\right|^{2}>} \\
= & \frac{\pi^{2}}{32} \sum_{d_{1}\left|m_{1}, d_{2}\right| m_{2}} \sum_{k_{1}, k_{2}} \sum_{t_{j}} a_{j}\left(k_{1}\left(k_{1}+\frac{m_{1}}{d_{1}}\right)\right) \overline{a_{j}\left(k_{2}\left(k_{2}+\frac{m_{2}}{d_{2}}\right)\right)} \widetilde{h}\left(t_{j}\right)+O\left(t_{j}^{-1-\epsilon}\right),
\end{aligned}
$$


where

$$
\widetilde{h}\left(t_{j}\right)=\frac{1}{t_{j}^{2}} H_{1}\left(t_{j}, d_{1} k_{1}, m_{1}\right) \overline{H_{2}\left(t_{j}, d_{2} k_{2}, m_{2}\right)}\left(e^{-\left(\left(t_{j}-T\right) / T^{1-\varepsilon}\right)^{2}}+e^{-\left(\left(t_{j}+T\right) / T^{1-\varepsilon}\right)^{2}}\right) .
$$

Applying Kuznetsov's formula [25] to the inner sum, we obtain

$$
\begin{aligned}
& \sum_{t_{j}} a_{j}\left(k_{1}\left(k_{1}+\frac{m_{1}}{d_{1}}\right)\right) \overline{a_{j}\left(k_{2}\left(k_{2}+\frac{m_{2}}{d_{2}}\right)\right)} \widetilde{h}\left(t_{j}\right) \\
= & \frac{\delta_{k_{1}\left(k_{1}+\frac{m_{1}}{d_{1}}\right), k_{2}\left(k_{2}+\frac{m_{2}}{d_{2}}\right)}}{\pi^{2}} \int_{-\infty}^{\infty} t \tanh (\pi t) \widetilde{h}(t) d t-\frac{2}{\pi} \int_{0}^{\infty} \frac{\widetilde{h}(t) d_{i t}\left(k_{1}^{2}+k_{1} m_{1} / d_{1}\right)}{|\zeta(1+2 i t)|^{2}} \\
& d_{i t}\left(k_{2}^{2}+k_{2} m_{2} / d_{2}\right) d t+\frac{2 i}{\pi} \sum_{c} c^{-1} S\left(k_{1}^{2}+k_{1} m_{1} / d_{1}, k_{2}^{2}+k_{2} m_{2} / d_{2} ; c\right) \\
& \int_{-\infty}^{\infty} J_{2 i t}\left(\frac{4 \pi \sqrt{\left(k_{1}^{2}+k_{1} m_{1} / d_{1}\right)\left(k_{2}^{2}+k_{2} m_{2} / d_{2}\right)}}{c}\right) t \frac{\widetilde{h}(t)}{\cosh (\pi t)} d t .
\end{aligned}
$$

Here

$$
S(m, n ; c)=\sum_{a d \equiv 1(\bmod c)} e\left(\frac{d m+a n}{c}\right)
$$

is the Kloosterman sum and

$$
d_{i t}(n)=\sum_{d_{1} d_{2}=n}\left(\frac{d_{1}}{d_{2}}\right)^{i t} .
$$

Thus, we have

$$
\begin{aligned}
& \sum_{t_{j}}\left(e^{-\left(\left(t_{j}-T\right) / T^{1-\varepsilon}\right)^{2}}+e^{-\left(\left(t_{j}+T\right) / T^{1-\varepsilon}\right)^{2}}\right) L\left(1, \operatorname{sym}^{2} \varphi_{j}\right) \\
& <P_{h_{1}, m_{1}},\left|\mu_{j}\right|^{2}>\overline{<P_{h_{2}, m_{2}},\left|\mu_{j}\right|^{2}>} \\
= & \frac{\pi^{2}}{32} \sum_{d_{1}, d_{2}, k_{1}, k_{2}}\left(\frac{\delta_{k_{1}\left(k_{1}+\frac{m_{1}}{d_{1}}\right), k_{2}\left(k_{2}+\frac{m_{2}}{d_{2}}\right)}}{\pi^{2}} \int_{-\infty}^{\infty} t \tanh (\pi t) \widetilde{h}(t) d t\right. \\
& -\frac{2}{\pi} \int_{0}^{\infty} \frac{\widetilde{h}(t)}{|\zeta(1+2 i t)|^{2}} d_{i t}\left(k_{1}^{2}+k_{1} m_{1} / d_{1}\right) d_{i t}\left(k_{2}^{2}+k_{2} m_{2} / d_{2}\right) d t \\
+ & \frac{2 i}{\pi} \sum_{c} c^{-1} S\left(k_{1}^{2}+k_{1} m_{1} / d_{1}, k_{2}^{2}+k_{2} m_{2} / d_{2} ; c\right) \\
& \left.\quad \int_{-\infty}^{\infty} J_{2 i t}\left(\frac{4 \pi \sqrt{\left(k_{1}^{2}+k_{1} m_{1} / d_{1}\right)\left(k_{2}^{2}+k_{2} m_{2} / d_{2}\right)}}{c}\right) t \frac{\widetilde{h}(t)}{\cosh (\pi t)} d t\right),
\end{aligned}
$$

Next, we will estimate these three terms respectively. 


\subsubsection{The Diagonal Term (2.5)}

Since $k_{1}\left(k_{1}+\frac{m_{1}}{d_{1}}\right)=k_{2}\left(k_{2}+\frac{m_{2}}{d_{2}}\right)$ has at most finitely many solutions if $m_{1} / d_{1} \neq m_{2} / d_{2}$, and the integer solutions to $k_{1}\left(k_{1}+\frac{m_{1}}{d_{1}}\right)=k_{2}\left(k_{2}+\frac{m_{2}}{d_{2}}\right)$ are only $k_{1}=k_{2}$ if $\frac{m_{1}}{d_{1}}=\frac{m_{2}}{d_{2}}$. Thus, the diagonal terms are

$$
\frac{\pi^{2}}{32} \int_{-\infty}^{\infty} \frac{\left(e^{-\left(\left(t_{j}-T\right) / T^{1-\varepsilon}\right)^{2}}+e^{-\left(\left(t_{j}+T\right) / T^{1-\varepsilon}\right)^{2}}\right)}{t} \sum_{m_{1} / d_{1}=m_{2} / d_{2}} \sum_{k \geq 1} H_{1}\left(t, d_{1} k, m_{1}\right) \bar{H}_{2}\left(t, d_{2} k, m_{2}\right) d t+O(1)
$$

where

$$
\begin{aligned}
& H_{1}\left(t, d_{1} k, m_{1}\right) \bar{H}_{2}\left(t, d_{2} k, m_{2}\right) \\
= & \int_{0}^{1} \int_{0}^{1} \frac{1}{\tau \eta(1-\tau)(1-\eta)} \cos \left(\frac{m_{1}}{d_{1} k} t(2 \tau-1)\right) \cos \left(\frac{m_{2}}{d_{2} k} t(2 \eta-1)\right) \\
& h_{1}\left(\frac{t \sqrt{\tau(1-\tau)}}{\pi d_{1} k \sqrt{1+\frac{2 \tau m_{1}}{d_{1} k}+\frac{\tau m_{1}^{2}}{d_{1}^{2} k^{2}}}}\right) h_{2}\left(\frac{t \sqrt{\eta(1-\eta)}}{\pi d_{2} k \sqrt{1+\frac{2 \eta m_{2}}{d_{2} k}+\frac{\eta m_{2}^{2}}{d_{2}^{2} k^{2}}}}\right) d \tau d \eta
\end{aligned}
$$

For $i=1,2$; restricting $h_{i}$ on $\mathbb{R}$ and $h_{i}$ satisfy $h_{i}^{(n)}(t) \ll(1+|t|)^{-N}$ for any $n>0$ sufficiently large $N$ and $h_{i}(t) \ll t^{10}$ when $t \rightarrow 0$. Thus, $h_{i}$ are continuous uniformly on $\mathbb{R}$. For the sum over $k$, we estimate it as

$$
\begin{aligned}
& \sum_{k \geq 1} H_{1}\left(t, d_{1} k, m_{1}\right) \bar{H}_{2}\left(t, d_{2} k, m_{2}\right) \\
& =\int_{0}^{1} \int_{0}^{1} \int_{0}^{\infty} \cos \left(\frac{m_{1}}{d_{1} k} t(2 \tau-1)\right) \cos \left(\frac{m_{2}}{d_{2} k} t(2 \eta-1)\right) h_{1}\left(\frac{t \sqrt{\tau(1-\tau)}}{\pi d_{1} k \sqrt{1+\frac{2 \tau m_{1}}{d_{1} k}+\frac{\tau m_{1}^{2}}{d_{1}^{2} k^{2}}}}\right) \\
& h_{2}\left(\frac{t \sqrt{\eta(1-\eta)}}{\pi d_{2} k \sqrt{1+\frac{2 \eta m_{2}}{d_{2} k}+\frac{\eta m_{2}^{2}}{d_{2}^{2} k^{2}}}}\right) d k \frac{1}{\tau \eta(1-\tau)(1-\eta)} d \tau d \eta+O(1) \\
& =\int_{0}^{1} \int_{0}^{1} \int_{0}^{\infty} \cos \left(\frac{m_{1}}{d_{1} k} t(2 \tau-1)\right) \cos \left(\frac{m_{2}}{d_{2} k} t(2 \eta-1)\right) h_{1}\left(\frac{t \sqrt{\tau(1-\tau)}}{\pi d_{1} k}\right) \\
& h_{2}\left(\frac{t \sqrt{\eta(1-\eta)}}{\pi d_{2} k}\right) d k \frac{1}{\tau \eta(1-\tau)(1-\eta)} d \tau d \eta+O(1) \\
& =\frac{t}{\pi} \int_{0}^{1} \int_{0}^{1} \int_{0}^{\infty} \frac{\cos \left(\frac{\pi m_{1}}{d_{1}} \xi(2 \tau-1)\right) \cos \left(\frac{\pi m_{2}}{d_{2}} \xi(2 \eta-1)\right)}{\tau \eta(1-\tau)(1-\eta)} h_{1}\left(\frac{\xi \sqrt{\tau(1-\tau)}}{d_{1}}\right)
\end{aligned}
$$




$$
h_{2}\left(\frac{\xi \sqrt{\eta(1-\eta)}}{d_{2}}\right) \frac{d \xi}{\xi^{2}} d \tau d \eta+O(1)
$$

Therefore, we obtain the main term of the diagonal term is

$$
\begin{array}{r}
\frac{\pi^{3 / 2}}{64} T^{(1-\epsilon)} \sum_{\frac{m_{1}}{d_{1}}=\frac{m_{2}}{d_{2}}} \int_{0}^{1} \int_{0}^{1} \int_{0}^{\infty} \frac{\cos \left(\frac{\pi m_{1}}{d_{1}} \xi(2 \tau-1)\right) \cos \left(\frac{\pi m_{2}}{d_{2}} \xi(2 \eta-1)\right)}{\tau \eta(1-\tau)(1-\eta)} \\
h_{1}\left(\frac{\xi \sqrt{\tau(1-\tau)}}{d_{1}}\right) h_{2}\left(\frac{\xi \sqrt{\eta(1-\eta)}}{d_{2}}\right) \frac{d \xi}{\xi^{2}} d \tau d \eta
\end{array}
$$

i.e.

$$
\begin{array}{r}
\frac{\pi^{3 / 2}}{64} T^{(1-\epsilon)} \sum_{m_{1} / d_{1}=m_{2} / d_{2}} \int_{0}^{\infty} \int_{0}^{1} \frac{\cos \left(\frac{\pi m_{1}}{d_{1}} \xi(2 \tau-1)\right) h_{1}\left(\frac{\xi \sqrt{\tau(1-\tau)}}{d_{1}}\right)}{\tau(1-\tau)} d \tau \\
\int_{0}^{1} \frac{\cos \left(\frac{\pi m_{2}}{d_{2}} \xi(2 \eta-1)\right) h_{2}\left(\frac{\xi \sqrt{\eta(1-\eta)}}{d_{2}}\right)}{\eta(1-\eta)} d \eta \frac{d \xi}{\xi^{2}} .
\end{array}
$$

For the holomorphic case [34], it is

$$
\frac{K \pi}{4} \int_{0}^{\infty} u(\xi) d \xi \sum_{m_{1} / d_{1}=m_{2} / d_{2}} \frac{1}{d_{1} d_{2}} \int_{0}^{\infty} h_{1}\left(d_{2} \eta\right) h_{2}\left(d_{1} \eta\right) \frac{d \eta}{\eta^{2}}
$$

\subsubsection{The Non-diagonal Term (2.7)}

The non-diagonal term is:

$$
\sum_{\substack{d_{1}\left|m_{1} \\ d_{2}\right| m_{2}}} \sum_{k_{1}, k_{2}} \sum_{c \geq 1} \frac{S\left(k_{1}\left(k_{1}+\frac{m_{1}}{d_{1}}\right), k_{2}\left(k_{2}+\frac{m_{2}}{d_{2}}\right) ; c\right)}{c} \int_{\mathbb{R}} J_{2 i t}\left(\frac{4 \pi \sqrt{k_{1} k_{2}\left(k_{1}+\frac{m_{1}}{d_{1}}\right)\left(k_{2}+\frac{m_{2}}{d_{2}}\right)}}{c}\right) \frac{\tilde{h}(t) t}{\cosh (\pi t)} d t
$$

where

$$
\begin{gathered}
\tilde{h}(t)=\frac{1}{t^{2}} H_{1}\left(t, d_{1} k_{1}, m_{1}\right) \overline{H_{2}\left(t, d_{2} k_{2}, m_{2}\right)}\left(e^{-\left((t-T) / T^{1-\varepsilon}\right)^{2}}+e^{-\left((t+T) / T^{1-\varepsilon}\right)^{2}}\right), \\
H_{j}(t, k, m)=\int_{0}^{1}\left(\frac{1+\frac{m}{k}}{1+\frac{2 \tau m}{k}+\frac{\tau m^{2}}{k^{2}}}\right)^{i t} \frac{1}{\tau(1-\tau)} h_{j}\left(\frac{t \sqrt{\tau(1-\tau)}}{\pi k \sqrt{1+\frac{2 \tau m}{k}+\frac{\tau m^{2}}{k^{2}}}}\right) d \tau
\end{gathered}
$$

for $j=1,2$. 
Let $x=\frac{4 \pi \sqrt{k_{1} k_{2}\left(k_{1}+\frac{m_{1}}{d_{1}}\right)\left(k_{2}+\frac{m_{2}}{d_{2}}\right)}}{c}$, the inner integral in the non-diagonal terms is

$$
I_{T}(x)=\frac{1}{2} \int_{\mathbb{R}} \frac{J_{2 i t}(x)-J_{-2 i t}(x)}{\sinh (\pi t)} \tilde{h}(t) \tanh \pi t d t
$$

Since $\tanh (\pi t)=\operatorname{sgn}(t)+O\left(e^{-\pi|t|}\right)$ for large $|t|$, we can remove $\tanh (\pi t)$ by getting a negligible term $O\left(T^{-N}\right)$ for any $N>0$.

Next we apply the Parseval identity and the Fourier transform in (Bateman, volume 1, p. 59)

$$
\left(\frac{J_{2 i t}(x) \widehat{J}_{-2 i t}(x)}{\sinh (\pi t)}\right)(y)=-i \cos (x \cosh (\pi y)) .
$$

Remark: This transform is very useful in the analysis of the automorphic forms recently, e.g. Liu and Ye [27] applied this in their proof for a subconvexity bound for Rankin-Selberg $L$-functions.

By the evaluation of the Fresnel integrals, we have

$$
\begin{aligned}
& I_{T}(x)=\frac{1}{2} \int_{\mathbb{R}}\left(\frac{J_{2 i t}(x) \widehat{-J}_{-2 i t}(x)}{\sinh (\pi t)}\right)(y) \widehat{(\tilde{h}(t) t)}(y) d y+O\left(T^{-N}\right) \\
& =\frac{-i}{2} \int_{\mathbb{R}} \cos (x \cosh (\pi y)) \widehat{(\tilde{h}(t) t)}(y) d y+O\left(T^{-N}\right) \\
& =\frac{-i}{2} \int_{\mathbb{R}} \cos \left(x+\frac{1}{2} \pi^{2} x y^{2}\right) \widehat{(\tilde{h}(t) t)}(y) d y+O\left(T^{-N}\right) \\
& =\frac{-i}{2} \int_{0}^{\infty} \tilde{h}\left(\sqrt{\frac{x y}{2}}\right) \sqrt{\frac{x y}{2}} \cos \left(x-y+\frac{\pi y}{4}\right) \frac{d y}{\sqrt{\pi y}}+O\left(T^{-N}\right) \\
& =\frac{-i}{2} \int_{0}^{\infty}\left(e^{-\left(\left(\sqrt{\frac{x y}{2}}-T\right) / T^{1-\epsilon}\right)^{2}}+e^{-\left(\left(\sqrt{\frac{x y}{2}}+T\right) / T^{1-\epsilon}\right)^{2}}\right) \sqrt{\frac{2}{x y}} \int_{0}^{1} \int_{0}^{1} \\
& \frac{\cos \left(\frac{m_{1}}{d_{1} k} \sqrt{\frac{x y}{2}}(2 \tau-1)\right) \cos \left(\frac{m_{2}}{d_{2} k} \sqrt{\frac{x y}{2}}(2 \eta-1)\right)}{\tau \eta(1-\tau)(1-\eta)} h_{1}\left(\frac{\sqrt{\frac{x y}{2}} \sqrt{\tau(1-\tau)}}{\pi d_{1} k \sqrt{1+\frac{2 \tau m_{1}}{d_{1} k}+\frac{\tau m_{1}^{2}}{d_{1}^{2} k^{2}}}}\right) \\
& h_{2}\left(\frac{\sqrt{\frac{x y}{2}} \sqrt{\eta(1-\eta)}}{\pi d_{2} k \sqrt{1+\frac{2 \eta m_{2}}{d_{2} k}+\frac{\eta m_{2}^{2}}{d_{2}^{2} k^{2}}}}\right) d \tau d \eta \cos \left(x-y+\frac{\pi}{4}\right) \frac{d y}{\sqrt{\pi y}}
\end{aligned}
$$

Thus, the non-diagonal terms are equal to 


$$
\begin{aligned}
& \frac{-i}{2} \sum_{\substack{d_{1}\left|m_{1} \\
d_{2}\right| m_{2}}} \sum_{k_{1}, k_{2}} \sum_{c \geq 1} \frac{S\left(k_{1}\left(k_{1}+\frac{m_{1}}{d_{1}}\right), k_{2}\left(k_{2}+\frac{m_{2}}{d_{2}}\right) ; c\right)}{c} \int_{0}^{\infty}\left(e^{-\left(\left(\sqrt{\frac{x y}{2}}-T\right) / T^{1-\epsilon}\right)^{2}}+\right. \\
& \left.e^{-\left(\left(\sqrt{\frac{x y}{2}}+T\right) / T^{1-\epsilon}\right)^{2}}\right) \sqrt{\frac{2}{x y}} \int_{0}^{1} \int_{0}^{1} \frac{\cos \left(\frac{m_{1}}{d_{1} k} \sqrt{\frac{x y}{2}}(2 \tau-1)\right) \cos \left(\frac{m_{2}}{d_{2} k} \sqrt{\frac{x y}{2}}(2 \eta-1)\right)}{\tau \eta(1-\tau)(1-\eta)} \\
& h_{1}\left(\frac{\sqrt{\frac{x y}{2}} \sqrt{\tau(1-\tau)}}{\pi d_{1} k_{1}}\right) h_{2}\left(\frac{\sqrt{\frac{x y}{2}} \sqrt{\eta(1-\eta)}}{\pi d_{2} k_{2}}\right) d \tau d \eta \cos \left(x-y+\frac{\pi}{4}\right) \frac{d y}{\sqrt{\pi y}} \\
& +O(1)
\end{aligned}
$$

Since both $h_{1}(t)$ and $h_{2}(t)$ satisfy $h_{i}^{(n)} \ll(1+|t|)^{-N}$ for any $n>0$ and sufficiently large $N$, and $h_{i}(t) \ll t^{10}$ when $t \rightarrow 0$, the above sum is concentrated on

$$
\begin{gathered}
\left|\frac{\sqrt{\frac{x y}{2}}-T}{T^{1-\epsilon}}\right| \ll T^{\frac{\epsilon}{2}} \\
T^{-\frac{1}{10}} \ll \frac{x y \tau(1-\tau)}{k_{1}^{2}} \ll T^{\epsilon} \\
T^{-\frac{1}{10}} \ll \frac{x y \eta(1-\eta)}{k_{2}^{2}} \ll T^{\epsilon}
\end{gathered}
$$

Thus we can get the following range

$$
\sqrt{\frac{x y}{2}} \sim T
$$

Note that here $x \sim k_{1} k_{2} c^{-1}$, the ranges for $k_{1}, k_{2}, c$ are as follows

$$
\begin{aligned}
T^{1-\frac{\epsilon}{2}} \sqrt{\tau(1-\tau)} & \ll k_{1} \ll T^{\frac{21}{20}+\epsilon} \sqrt{\tau(1-\tau)}, \\
T^{1-\frac{\epsilon}{2}} \sqrt{\eta(1-\eta)} & \ll k_{2} \ll T^{\frac{21}{20}+\epsilon} \sqrt{\eta(1-\eta)}, \\
c & \ll y T^{\frac{1}{10}}
\end{aligned}
$$

(In the holomorphic case [34], one could get $k_{1} \sim k_{2} \sim T$ and $y \ll c$, so by partial integration, the terms with $c \gg T^{\epsilon}$ contribute $O(1)$.)

Here by the above relations and partial integration sufficiently many times, we will get sufficiently large power of $y, k_{1}$ and $k_{2}$ occurring in the denominator, so we get the terms with $c \gg T^{\frac{1}{10}+\epsilon}$ contribute $O(1)$. 
Denote the sum (2.10) as

$$
\sum_{\substack{d_{1}\left|m_{1} \\ d_{2}\right| m_{2}}} \sum_{k_{1}, k_{2}} \sum_{c \geq 1} \frac{S\left(k_{1}\left(k_{1}+\frac{m_{1}}{d_{1}}\right), k_{2}\left(k_{2}+\frac{m_{2}}{d_{2}}\right) ; c\right)}{c} J_{k_{1}, k_{2}, c}+O(1) .
$$

Making the change of variable $t=\frac{\sqrt{\frac{x y}{2}}}{T}$, we get $J_{k_{1}, k_{2}, c}$ is

$$
\begin{aligned}
& \frac{2^{\frac{3}{2}}}{\sqrt{\pi x}} \int_{0}^{\infty}\left(e^{-\left(\frac{(t-1)}{T^{-\epsilon}}\right)^{2}}+e^{-\left(\frac{(t+1)}{T^{-\epsilon}}\right)^{2}}\right) \frac{1}{t} \sin \left(-x+\frac{2(t T)^{2}}{x}-\frac{\pi}{4}\right) \int_{0}^{1} \int_{0}^{1} \frac{\cos \left(\frac{m_{1}}{d_{1} k} t T(2 \tau-1)\right)}{\tau(1-\tau)} \\
& \frac{\cos \left(\frac{m_{2}}{d_{2} k} t T(2 \eta-1)\right)}{\eta(1-\eta)} h_{1}\left(\frac{t T \sqrt{\tau(1-\tau)}}{\pi d_{1} k_{1}}\right) h_{2}\left(\frac{t T \sqrt{\eta(1-\eta)}}{\pi d_{2} k_{2}}\right) d \tau d \eta d t
\end{aligned}
$$

By Taylor expansion,

$$
\begin{aligned}
x i & =\frac{4 \pi i}{c} \sqrt{k_{1} k_{2}\left(k_{1}+\frac{m_{1}}{d_{1}}\right)\left(k_{2}+\frac{m_{2}}{d_{2}}\right)} \\
& =\frac{2 \pi i}{c}\left(2 k_{1} k_{2}+\frac{m_{2} k_{1}}{d_{2}}+\frac{m_{1} k_{2}}{d_{1}}+\cdots\right)
\end{aligned}
$$

So we can write

$$
J_{k_{1}, k_{2}, c}=\operatorname{Im}\left(e_{c}\left(-\left(2 k_{1} k_{2}+\frac{m_{2} k_{1}}{d_{2}}+\frac{m_{1} k_{2}}{d_{1}}\right)\right) f_{c}\left(k_{1}, k_{2}\right)\right),
$$

where

$$
\begin{aligned}
f_{c}\left(k_{1}, k_{2}\right)= & e_{c}\left(\frac{m_{1} m_{2}}{2 d_{1} d_{2}}-\frac{m_{1}^{2} k_{2}}{4 d_{1}^{2} k_{1}}-\frac{m_{2}^{2} k_{1}}{4 d_{2}^{2} k_{2}}+\cdots\right) \frac{2^{\frac{3}{2}}}{\sqrt{\pi x}} \int_{0}^{\infty}\left(e^{-\left(\frac{(t-1)}{\left.T^{-\epsilon}\right)^{2}}+e^{-\left(\frac{(t+1)}{T^{-\epsilon}}\right)^{2}}\right) \frac{1}{t}}\right. \\
& e^{i\left(\frac{2(t T)^{2}}{x}-\frac{\pi}{4}\right)} \int_{0}^{1} \int_{0}^{1} \frac{\cos \left(\frac{m_{1}}{d_{1} k} t T(2 \tau-1)\right) \cos \left(\frac{m_{2}}{d_{2} k} t T(2 \eta-1)\right)}{\tau \eta(1-\tau)(1-\eta)} \\
& h_{1}\left(\frac{t T \sqrt{\tau(1-\tau)}}{\pi d_{1} k_{1}}\right) h_{2}\left(\frac{t T \sqrt{\eta(1-\eta)}}{\pi d_{2} k_{2}}\right) d \tau d \eta d t
\end{aligned}
$$

and we use the notation $e_{c}(z)=e^{\frac{2 \pi i z}{c}}$.

Reducing the summation over $k_{1}, k_{2}$ into congruence classes mod $c$, we have,

$$
\sum_{k_{1}, k_{2} \geq 1} S\left(k_{1}\left(k_{1}+\frac{m_{1}}{d_{1}}\right), k_{2}\left(k_{2}+\frac{m_{2}}{d_{2}}\right) ; c\right) e_{c}\left(-\left(2 k_{1} k_{2}+\frac{m_{2} k_{1}}{d_{2}}+\frac{m_{1} k_{2}}{d_{1}}\right)\right) f_{c}\left(k_{1}, k_{2}\right)
$$




$$
\begin{aligned}
= & \sum_{a, b \bmod c} S\left(a\left(a+\frac{m_{1}}{d_{1}}\right), b\left(b+\frac{m_{2}}{d_{2}}\right) ; c\right) e_{c}\left(-\left(2 a b+\frac{m_{2} a}{d_{2}}+\frac{m_{1} b}{d_{1}}\right)\right) \\
& \sum_{k_{1} \equiv a, k_{2} \equiv b \bmod c} f_{c}\left(k_{1}, k_{2}\right) \\
= & \frac{1}{c^{2}} \sum_{u, v \bmod c a, b \bmod c} S\left(a\left(a+\frac{m_{1}}{d_{1}}\right), b\left(b+\frac{m_{2}}{d_{2}}\right) ; c\right) \\
& e_{c}\left(-\left(2 a b+\left(\frac{m_{2}}{d_{2}}+u\right) a+\left(\frac{m_{1}}{d_{1}}+v\right) b\right)\right)\left(\sum_{k_{1}, k_{2}} f_{c}\left(k_{1}, k_{2}\right) e_{c}\left(-u k_{1}-v k_{2}\right)\right) .
\end{aligned}
$$

Apply the Poisson summation for the sum in $k_{1}, k_{2}$ and obtain,

$$
\sum_{k_{1}, k_{2}} f_{c}\left(k_{1}, k_{2}\right) e_{c}\left(-u k_{1}-v k_{2}\right)=\sum_{l_{1}, l_{2}} \iint_{\mathrm{R}^{2}} f_{c}\left(k_{1}, k_{2}\right) e\left(\left(l_{1}-\frac{u}{c}\right) k_{1}+\left(l_{2}-\frac{v}{c}\right) k_{2}\right) d k_{1} d k_{2} .
$$

We can assume $|u| \leq \frac{c}{2},|v| \leq \frac{c}{2}$, by partial integration sufficiently many times, we get

$$
\sum_{k_{1}, k_{2}} f_{c}\left(k_{1}, k_{2}\right) e_{c}\left(-u k_{1}-v k_{2}\right)=\iint_{\mathrm{R}^{2}} f_{c}\left(k_{1}, k_{2}\right) e\left(-\frac{u}{c} k_{1}-\frac{v}{c} k_{2}\right) d k_{1} d k_{2}+O\left(T^{-A}\right)
$$

for any $A>1$. For $(u, v) \neq(0,0)$, by partial integration sufficiently many times, we also obtain

$$
\iint_{\mathrm{R}^{2}} f_{c}\left(k_{1}, k_{2}\right) e\left(-\frac{u}{c} k_{1}-\frac{v}{c} k_{2}\right) d k_{1} d k_{2} \ll T^{-A},
$$

for any $A>0$. Thus only $(u, v)=(0,0)$ contributes. We can allow $c \gg T^{\frac{1}{10}+\epsilon}$ in the $c$-summation, notice that we have the term $\frac{T^{2} c}{k_{1} k_{2}}$ in $f_{c}\left(k_{1}, k_{2}\right)$, so by partial integration sufficiently many times,

$$
\iint_{\mathrm{R}^{2}} f_{c}\left(k_{1}, k_{2}\right) d k_{1} d k_{2} \ll c^{-A} T^{2}
$$

for any $A>0$. For fixed $d_{i}, m_{i}(i=1,2)$, denote

$$
S_{c}=\sum_{a, b \bmod c} S\left(a\left(a+\frac{m_{1}}{d_{1}}\right), b\left(b+\frac{m_{2}}{d_{2}}\right) ; c\right) e_{c}\left(-\left(2 a b+\frac{m_{2} a}{d_{2}}+\frac{m_{1} b}{d_{1}}\right)\right)
$$


Thus, the non-diagonal contribution is

$$
\begin{aligned}
& \sum_{\substack{d_{1}\left|m_{1} \\
d_{2}\right| m_{2}}} \sum_{c \geq 1} \operatorname{Im}\left(\frac{S_{c}}{c^{2}} \iint_{\mathrm{R}^{2}} f_{c}\left(k_{1}, k_{2}\right) d k_{1} d k_{2}\right)+O(1) \\
& =\sum_{\substack{d_{1}\left|m_{1} \\
d_{2}\right| m_{2}}} \sum_{c \geq 1} \operatorname{Im}\left(\frac{S_{c}}{c^{2}} \iint_{\mathrm{R}^{2}} e_{c}\left(\frac{m_{1} m_{2}}{2 d_{1} d_{2}}-\frac{m_{1}^{2} k_{2}}{4 d_{1}^{2} k_{1}}-\frac{m_{2}^{2} k_{1}}{4 d_{2}^{2} k_{2}}\right) \frac{2^{\frac{3}{2}}}{\sqrt{\pi x}} \int_{0}^{\infty}\left(e^{-\left(\frac{(t-1)}{T^{-\epsilon}}\right)^{2}}+e^{-\left(\frac{(t+1)}{T^{-\epsilon}}\right)^{2}}\right) \frac{1}{t}\right. \\
& e^{i\left(\frac{2(t T)^{2}}{x}-\frac{\pi}{4}\right)} \int_{0}^{1} \int_{0}^{1} \frac{\cos \left(\frac{m_{1}}{d_{1} k_{1}} t T(2 \tau-1)\right) \cos \left(\frac{m_{2}}{d_{2} k_{2}} t T(2 \eta-1)\right)}{\tau \eta(1-\tau)(1-\eta)} h_{1}\left(\frac{t T \sqrt{\tau(1-\tau)}}{\pi d_{1} k_{1}}\right) \\
& \left.h_{2}\left(\frac{t T \sqrt{\eta(1-\eta)}}{\pi d_{2} k_{2}}\right) d \tau d \eta d t d k_{1} d k_{2}\right)+O(1) \\
& =T \sum_{\substack{d_{1}\left|m_{1} \\
d_{2}\right| m_{2}}} \sum_{c \geq 1} \operatorname{Im}\left(\frac { S _ { c } \zeta _ { 8 } } { c ^ { \frac { 3 } { 2 } } } \int \int _ { \mathrm { R } ^ { 2 } } e _ { c } ( \frac { m _ { 1 } m _ { 2 } } { 2 d _ { 1 } d _ { 2 } } - \frac { m _ { 1 } ^ { 2 } \phi } { 4 d _ { 1 } ^ { 2 } \xi } - \frac { m _ { 2 } ^ { 2 } \xi } { 4 d _ { 2 } ^ { 2 } \phi } ) \frac { 2 ^ { \frac { 3 } { 2 } } } { \sqrt { \pi } ( \xi \phi ) ^ { \frac { 3 } { 2 } } } \int _ { 0 } ^ { \infty } \left(e^{-\left(\frac{(t-1)}{T^{-\epsilon}}\right)^{2}}+\right.\right. \\
& \left.e^{-\left(\frac{(t+1)}{T^{-\epsilon}}\right)^{2}}\right) e(\xi \phi c) \int_{0}^{1} \int_{0}^{1} \frac{\cos \left(\frac{m_{1} \xi}{d_{1}}(2 \tau-1)\right) \cos \left(\frac{m_{2} \phi}{d_{2}}(2 \eta-1)\right)}{\tau \eta(1-\tau)(1-\eta)} h_{1}\left(\frac{\xi \sqrt{\tau(1-\tau)}}{\pi d_{1}}\right) \\
& \left.h_{2}\left(\frac{\phi \sqrt{\eta(1-\eta)}}{\pi d_{2}}\right) d \tau d \eta d t d \xi d \phi\right)+O(1) \\
& =T^{1-\epsilon} \sum_{\substack{d_{1}\left|m_{1} \\
d_{2}\right| m_{2}}} \sum_{c \geq 1} \operatorname{Im}\left(\frac{S_{c} \zeta_{8}}{c^{\frac{3}{2}}} \iint_{\mathrm{R}^{2}} e_{c}\left(\frac{m_{1} m_{2}}{2 d_{1} d_{2}}-\frac{m_{1}^{2} \phi}{4 d_{1}^{2} \xi}-\frac{m_{2}^{2} \xi}{4 d_{2}^{2} \phi}\right) \frac{2^{\frac{3}{2}}}{(\xi \phi)^{\frac{3}{2}}}\right. \\
& e(\xi \phi c) \int_{0}^{1} \int_{0}^{1} \frac{\cos \left(\frac{m_{1} \xi}{d_{1}}(2 \tau-1)\right) \cos \left(\frac{m_{2} \phi}{d_{2}}(2 \eta-1)\right)}{\tau \eta(1-\tau)(1-\eta)} h_{1}\left(\frac{\xi \sqrt{\tau(1-\tau)}}{\pi d_{1}}\right) \\
& \left.h_{2}\left(\frac{\phi \sqrt{\eta(1-\eta)}}{\pi d_{2}}\right) d \tau d \eta d \xi d \phi\right)+O(1)
\end{aligned}
$$

\subsubsection{The Continuous Spectrum Part (2.6)}

Next, we study the continuous spectrum integrals, which is

$$
\sum_{\substack{d_{1}\left|m_{1} \\ d_{2}\right| m_{2}}} \sum_{k_{1}, k_{2} \geq 1} \int_{0}^{\infty} \frac{\tilde{h}(t)}{|\zeta(1+2 i t)|^{2}} d_{i t}\left(k_{1}^{2}+k_{1} m_{1} / d_{1}\right) d_{i t}\left(k_{2}^{2}+k_{2} m_{2} / d_{2}\right) d t
$$


Recall that $|\zeta(1+2 i t)| \geq c \log ^{-2 / 3}(2+|t|)$ for some $c>0[47]$,

$$
d_{i t}(n)=\sum_{d_{1} d_{2}=n}\left(\frac{d_{1}}{d_{2}}\right)^{i t}=O\left(n^{\epsilon}\right),
$$

and

$$
\begin{gathered}
\tilde{h}(t)=\frac{1}{t^{2}} H_{1}\left(t, d_{1} k_{1}, m_{1}\right) \overline{H_{2}\left(t, d_{2} k_{2}, m_{2}\right)}\left(e^{-\left(\frac{t-T}{T^{1-\epsilon}}\right)^{2}}+e^{-\left(\frac{t+T}{T^{1-\epsilon}}\right)^{2}}\right), \\
H_{j}(t, k, m)=\int_{0}^{1}\left(\frac{1+\frac{m}{k}}{1+\frac{2 \tau m}{k}+\frac{\tau m^{2}}{k^{2}}}\right)^{i t} \frac{1}{\tau(1-\tau)} h_{j}\left(\frac{t \sqrt{\tau(1-\tau)}}{\pi k \sqrt{1+\frac{2 \tau m}{k}+\frac{\tau m^{2}}{k^{2}}}}\right) d \tau
\end{gathered}
$$

for $j=1,2$.

The Fourier expansion of $E\left(z, \frac{1}{2}+i t\right)$ is

$$
E\left(z, \frac{1}{2}+i t\right)=y^{\frac{1}{2}+i t}+\varphi\left(\frac{1}{2}+i t\right) y^{\frac{1}{2}-i t}+\frac{2 y^{\frac{1}{2}}}{\xi(1+2 i t)} \sum_{n=1}^{\infty} d_{i t}(n) K_{i t}(2 \pi n y) \cos (2 \pi n x)
$$

where

$$
\begin{gathered}
\varphi\left(\frac{1}{2}+i t\right)=\frac{\xi(2 i t)}{\xi(1+2 i t)} \\
\xi(s)=\pi^{-\frac{s}{2}} \Gamma\left(\frac{s}{2}\right) \zeta(s) .
\end{gathered}
$$

Thus, we have

$$
\begin{aligned}
<P_{h, m},\left|\mu_{t}\right|^{2}> & =\int_{\Gamma \backslash \mathcal{H}}\left|E\left(z, \frac{1}{2}+i t\right)\right|^{2}\left(\sum_{\gamma \in \Gamma_{\infty} \backslash \Gamma} h(y(\gamma z)) e(m x(\gamma z))\right) d \mu(z) \\
& =\sum_{k \neq 0,-m} d_{i t}(k) d_{i t}(k+m) \int_{0}^{\infty} K_{i t_{j}}(r) K_{i t_{j}}\left(\left|1+\frac{m}{k}\right| r\right) h\left(\frac{r}{2 \pi|k|}\right) \frac{d r}{r}
\end{aligned}
$$

Then, by the similar calculation in Section 2.1, we obtain the continuous spectrum integral is

$$
\int_{0}^{\infty}\left(e^{-\left(\frac{t-T}{T^{1-\epsilon}}\right)^{2}}+e^{-\left(\frac{t+T}{T^{1-\epsilon}}\right)^{2}}\right)|\zeta(1+2 i t)|^{2}<P_{h_{1}, m_{1}},\left|\mu_{t}\right|^{2}>\overline{<P_{h_{2}, m_{2}},\left|\mu_{t}\right|^{2}>} d t .
$$

Remark 2.1. We notice that the continuous spectrum part is not negligible and contributes to the main term, but in general, it is small in the cuspidal subspace.

For the inner product $<P_{h, m},\left|\mu_{t}\right|^{2}>$, if $m=0$, by the unfolding technique and Bessel identity, we have 


$$
\begin{aligned}
<P_{h, 0},\left|\mu_{t}\right|^{2}>= & \int_{\Gamma \backslash \mathcal{H}}\left|E\left(z, \frac{1}{2}+i t\right)\right|^{2}\left(\sum_{\gamma \in \Gamma_{\infty} \backslash \Gamma} h(y(\gamma z))\right) d \mu(z) \\
= & \frac{1}{2 \pi i} \int_{\Gamma \backslash \mathcal{H}} \int_{\mathfrak{R}(s)=2} H(s) E(z, s) d s\left|E\left(z, \frac{1}{2}+i t\right)\right|^{2} d \mu(z) \\
= & \frac{1}{2 \pi i} \int_{0}^{\infty} \int_{\mathfrak{R}(s)=2} H(s) y^{s} d s \int_{0}^{1}\left|E\left(z, \frac{1}{2}+i t\right)\right|^{2} d \frac{d x d y}{y^{2}} \\
= & \frac{1}{2 \pi i} \int_{0}^{\infty} \int_{\mathfrak{R}(s)=2} H(s) y^{s} d s\left(\left|y^{\frac{1}{2}+i t}+\varphi\left(\frac{1}{2}+i t\right) y^{\frac{1}{2}-i t}\right|^{2}\right. \\
& \left.+\frac{2}{|\xi(1+2 i t)|^{2}} \sum_{n=1}^{\infty} y\left|\sigma_{-2 i t}(n) K_{i t}(2 \pi n y)\right|^{2}\right) \frac{d y}{y^{2}},
\end{aligned}
$$

where $H(s)$ is the Mellin transform of $h(y)$,

$$
H(s)=\int_{0}^{\infty} h(y) y^{-s-1} d y
$$

Since $\left|\varphi\left(\frac{1}{2}+i t\right)\right|^{2}=1$, the first term of $(2.8)$ contributes

$$
2 \int_{0}^{\infty} h(y) \frac{d y}{y}
$$

and a rapidly decreasing function of $t$ as $t \rightarrow \infty$. We denote the second term of (2.8) as

$$
\left.I(t)=\frac{1}{\pi i|\xi(1+2 i t)|^{2}} \int_{\mathfrak{R}(s)=2} H(s)\left(\sum_{n=1}^{\infty} \frac{\left|\sigma_{-2 i t}(n)\right|^{2}}{n^{s}}\right) \int_{0}^{\infty}\left|K_{i t}(2 \pi y)\right|^{2}\right) y^{s-1} d y .
$$

The series inside can be evaluated as was the work of Ramanujan [37],

$$
\sum_{n=1}^{\infty} \frac{\left|\sigma_{-2 i t}(n)\right|^{2}}{n^{s}}=\frac{\zeta^{2}(s) \zeta(s-2 i t) \zeta(s+2 i t)}{\zeta(2 s)} .
$$

Hence, combined with the evaluation of the $K$-Bessel functions [11],

$$
\left.\int_{0}^{\infty}\left|K_{i t}(2 \pi y)\right|^{2}\right) y^{s-1} d y=\frac{\Gamma^{2}\left(\frac{s}{2}\right) \Gamma\left(\frac{s}{2}-i t\right) \Gamma\left(\frac{s}{2}+i t\right)}{\Gamma(s)}
$$

we have

$$
I(t)=\frac{2}{\pi i|\xi(1+2 i t)|^{2}} \int_{\mathfrak{R}(s)=2} \frac{\pi^{-s} H(s) \zeta^{2}(s) \zeta(s-2 i t) \zeta(s+2 i t) \Gamma^{2}\left(\frac{s}{2}\right) \Gamma\left(\frac{s}{2}-i t\right) \Gamma\left(\frac{s}{2}+i t\right)}{\zeta(2 s) \Gamma(s)} d s .
$$


Denote the above integrand as $B(s)$ and shift the above integral to $\mathfrak{R}(s)=\frac{1}{2}$, we have

$$
I(t)=\frac{4 \pi i}{\pi i|\xi(1+2 i t)|^{2}} \operatorname{Res}_{s=1} B(s)+\frac{2}{\pi i|\xi(1+2 i t)|^{2}} \int_{\mathfrak{R}(s)=\frac{1}{2}} B(s) d s+O\left(t^{-20}\right) .
$$

The error term comes from the contribution of poles at $s=1 \pm 2 i t$ of $\zeta(s \mp 2 i t)$ and the fact that $H(s)$ is rapidly decreasing in $\mathfrak{I m}(s)$. Applying Weyl's bound [50]

$$
\zeta\left(\frac{1}{2}+i t\right) \ll t^{\frac{1}{6}+\epsilon}
$$

and Stirling's formula

$$
|\Gamma(\sigma+i t)| \sim(2 \pi)^{1 / 2} t^{\sigma-\frac{1}{2}} e^{-\pi|t| / 2}
$$

we have

$$
\frac{2}{\pi i|\xi(1+2 i t)|^{2}} \int_{\mathfrak{R}(s)=\frac{1}{2}} B(s) d s \ll t^{-\frac{1}{6}+\epsilon} .
$$

For the residue term, we can write $B(s)$ as $\zeta^{2}(s) G(s)$ where

$$
G(s)=\frac{\pi^{-s} H(s) \zeta(s-2 i t) \zeta(s+2 i t) \Gamma^{2}\left(\frac{s}{2}\right) \Gamma\left(\frac{s}{2}-i t\right) \Gamma\left(\frac{s}{2}+i t\right)}{\zeta(2 s) \Gamma(s)}
$$

is holomorphic at $s=1$. Thus $\operatorname{Res}_{s=1} B(s)=2 \gamma G(1)+G^{\prime}(1)$. We can calculate that

$$
\begin{gathered}
G(1)=\frac{24}{\pi} H(1)=\frac{24}{\pi} \int_{0}^{\infty} h(y) \frac{d y}{y^{2}}=\int_{\Gamma \backslash \mathcal{H}} P_{h, 0} d \mu(z)=0, \\
G^{\prime}(1)=H^{\prime}(1) \frac{\pi^{-1} \zeta(1-2 i t) \zeta(1+2 i t) \Gamma^{2}\left(\frac{1}{2}\right) \Gamma\left(\frac{1}{2}-i t\right) \Gamma\left(\frac{1}{2}+i t\right)}{\zeta(2)} .
\end{gathered}
$$

From Stirling's formula

$$
\Gamma\left(\frac{1}{2}+2 i t\right) \sim(2 \pi)^{1 / 2} e^{-\pi|t|}
$$

and the arithmetic estimate [47]

$$
(\log t)^{-1} \ll \zeta(1+i t) \ll \log t
$$

the residue term

$$
\frac{4 \pi i}{\pi i|\xi(1+2 i t)|^{2}} \operatorname{Res}_{s=1} B(s)=\frac{24}{\pi} H^{\prime}(1)=\frac{24}{\pi} \int_{0}^{\infty} h(y) \log y \frac{d y}{y^{2}} .
$$


We obtain

$$
<P_{h, 0},\left|\mu_{t}\right|^{2}>=2 \int_{0}^{\infty} h(y) \frac{d y}{y}+\frac{24}{\pi} \int_{0}^{\infty} h(y) \log y \frac{d y}{y^{2}}+O\left(t^{-\frac{1}{6}}\right)
$$

if $\int_{0}^{\infty} h(y) \frac{d y}{y^{2}}=0$ or $P_{h, 0} \in C_{0,0}^{\infty}(X)$.

We can easily choose $h(y) \in C^{\infty}\left(R^{+}\right)$, such that the above main term is nonzero and $\int_{0}^{\infty} h(y) \frac{d y}{y^{2}}=0$. For example, let $h(y)=y^{2} C(y)$ where $C(y)$ is a step function such that $\int_{0}^{\infty} C(y) d y=0$ and $\int_{0}^{\infty} C(y)(y+\log y) d y \neq 0$, then apply approximation argument to obtain a smooth function which satisfies the same condition.

Therefore, we conclude that the above continuous spectrum integral also contributes $c T^{1-\varepsilon}$.

In Chapter 5 , if we restrict $B$ to the Maass-Hecke cusp forms, we can show the continuous spectrum integral is small enough so we can obtain the first part of main Theorem 1.4. 


\subsection{The Asymptotic Formula for the Variance}

From last section's results, we obtain the following:

Proposition 2.1. Let $\varphi_{j}$ denote the $j$-th Maass-Hecke eigenform with Laplacian eigenvalues $\lambda_{j}=\frac{1}{4}+t_{j}^{2}$. Denote $\left|\mu_{t}\right|^{2}$ be the measure $\left|E\left(z, \frac{1}{2}+i t\right)\right|^{2} \frac{d x d y}{y^{2}}$. For $m_{1}, m_{2} \neq$ $0, h_{1}, h_{2}$ be even analytic functions satisfying $h_{i}^{(n)}(t) \ll(1+|t|)^{-N}$ for any $n>0$ and sufficiently large $N, h_{i}(t) \ll t^{10}$ when $t \rightarrow 0, i=1,2$, fix $\epsilon>0$, we have

$$
\begin{aligned}
& \sum_{t_{j}}\left(e^{-\left(\frac{t_{j}-T}{T^{1-\epsilon}}\right)^{2}}+e^{-\left(\frac{t_{j}+T}{T^{1-\epsilon}}\right)^{2}}\right) L\left(1, \operatorname{sym}^{2} \varphi_{j}\right)<P_{h_{1}, m_{1}},\left|\mu_{j}\right|^{2}>\overline{<P_{h_{2}, m_{2}},\left|\mu_{j}\right|^{2}>} \\
+ & \int_{0}^{\infty}\left(e^{-\left(\frac{t-T}{T^{1-\epsilon}}\right)^{2}}+e^{-\left(\frac{t+T}{T^{1-\epsilon}}\right)^{2}}\right)|\zeta(1+2 i t)|^{2}<P_{h_{1}, m_{1}},\left|\mu_{t}\right|^{2}>\overline{<P_{h_{2}, m_{2}},\left|\mu_{t}\right|^{2}>} d t \\
= & T^{1-\epsilon} B\left(P_{h_{1}, m_{1}}, P_{h_{2}, m_{2}}\right)+O\left(T^{\frac{1}{2}-\epsilon}\right),
\end{aligned}
$$

where

$$
\begin{aligned}
& B\left(P_{h_{1}, m_{1}}, P_{h_{2}, m_{2}}\right) \\
= & \frac{\pi^{3 / 2}}{64} \sum_{m_{1} / d_{1}=m_{2} / d_{2}} \int_{0}^{\infty} \int_{0}^{1} \frac{\cos \left(\frac{\pi m_{1}}{d_{1}} \xi(2 \tau-1)\right) h_{1}\left(\frac{\xi \sqrt{\tau(1-\tau)}}{d_{1}}\right)}{\tau(1-\tau)} d \tau \\
& \int_{0}^{1} \frac{\cos \left(\frac{\pi m_{2}}{d_{2}} \xi(2 \eta-1)\right) h_{2}\left(\frac{\xi \sqrt{\eta(1-\eta)}}{d_{2}}\right)}{\eta(1-\eta)} d \eta \frac{d \xi}{\xi^{2}}+ \\
& \sum_{d_{1} \mid m_{1}} \sum_{c \geq 1} \operatorname{Im}\left(\frac{S_{c} \zeta_{8}}{c^{\frac{3}{2}}} \iint_{\mathrm{R}^{2}} e_{c}\left(\frac{m_{1} m_{2}}{2 d_{1} d_{2}}-\frac{m_{1}^{2} \xi}{4 d_{1}^{2} \phi}-\frac{m_{2}^{2} \phi}{4 d_{2}^{2} \xi}\right) \frac{2^{\frac{3}{2}}}{(\xi \phi)^{\frac{3}{2}}}\right. \\
& e\left(\left(d_{1} d_{2}\right)^{2} \xi \phi c\right) \int_{0}^{1} \int_{0}^{1} \frac{\cos \left(\pi m_{1} d_{2} \xi(2 \tau-1)\right) \cos \left(\pi m_{2} d_{1} \phi(2 \eta-1)\right)}{\tau \eta(1-\tau)(1-\eta)} \\
& \left.h_{1}\left(\xi d_{2} \sqrt{\tau(1-\tau)}\right) h_{2}\left(\phi d_{1} \sqrt{\eta(1-\eta)}\right) d \tau d \eta d \xi d \phi\right)
\end{aligned}
$$

Next, we remove the restriction on $h_{1}$ and $h_{2}$ by using Fourier transformation so that we can obtain a more general asymptotic formula for the quantum variance sum $S_{\psi}(\lambda)$. 
For any $\varphi(x)$ smooth function with compact support on $\mathbf{R}^{+}$, write $\varphi(x)=e^{-x^{2}} x^{A} \psi(x)$, where $A$ is a sufficiently large even integer, thus, by Fourier inversion,

$$
\varphi(x)=e^{-x^{2}} x^{A} \int_{-\infty}^{\infty} \hat{\psi}(y) e(x y) d y=\int_{-\infty}^{\infty} \hat{\psi}(y)\left(e(x y) e^{-x^{2}} x^{A}\right) d y
$$

where

$$
\hat{\psi}(y)=\int_{-\infty}^{\infty} \psi(x) e(-x y) d x
$$

since $\psi(x) \in C_{0}^{\infty}(0, \infty), \hat{\psi}(y)$ is analytic. $h_{t}(x)=e(x t) e^{-x^{2}} x^{A}$ satisfy the condition of the Proposition 2.1 for each $t$, so we have

$$
\begin{aligned}
& \sum_{t_{j}}\left(e^{-\left(\frac{t_{j}-T}{T^{1-\epsilon}}\right)^{2}}+e^{-\left(\frac{t_{j}+T}{T^{1-\epsilon}}\right)^{2}}\right) L\left(1, \operatorname{sym}^{2} \varphi_{j}\right)\left\langle P_{h, m_{1}},\left|\mu_{j}\right|^{2}\right\rangle \overline{\left\langle P_{h, m_{2}},\left|\mu_{j}\right|^{2}\right\rangle} \\
& +\int_{0}^{\infty}\left(e^{-\left(\frac{t-T}{T^{1-\epsilon}}\right)^{2}}+e^{-\left(\frac{t+T}{T^{1-\epsilon}}\right)^{2}}\right)|\zeta(1+2 i t)|^{2}\left\langle P_{h_{1}, m_{1}},\left|\mu_{t}\right|^{2}\right\rangle \overline{\left\langle P_{h_{2}, m_{2}},\left|\mu_{t}\right|^{2}\right\rangle} d t \\
= & T^{1-\epsilon} B\left(P_{h, m_{1}}, P_{h, m_{2}}\right)+O\left(T^{\frac{1}{2}-\epsilon}\right) .
\end{aligned}
$$

We also have

$$
\begin{aligned}
P_{\varphi, m}(z) & =\sum_{\gamma \in \Gamma_{\infty} \backslash \Gamma} \varphi(y(\gamma z)) e(m x(\gamma z)) \\
& =\sum_{\gamma \in \Gamma_{\infty} \backslash \Gamma}\left(\int_{-\infty}^{\infty} \hat{\psi}(t) e(t y(\gamma z)) e^{-(y(\gamma z))^{2}}(y(\gamma z))^{A} d t\right) e(m x(\gamma z)) \\
& =\int_{-\infty}^{\infty} \hat{\psi}(t) \sum_{\gamma \in \Gamma_{\infty} \backslash \Gamma}\left(e(t y(\gamma z)) e^{-(y(\gamma z))^{2}}(y(\gamma z))^{A}\right) e(m x(\gamma z)) d t \\
& =\int_{-\infty}^{\infty} \hat{\psi}(t) P_{e(x t) e^{-x^{2} x^{A}, m}} d t
\end{aligned}
$$

Thus, we have

$$
\left\langle P_{\varphi, m},\left|\mu_{j}\right|^{2}\right\rangle=\int_{-\infty}^{\infty} \hat{\psi}(t)\left\langle P_{e(x t) e^{-x^{2} x^{A}, m}},\left|\mu_{j}\right|^{2}\right\rangle d t
$$

So, for any $\varphi_{1}, \varphi_{2}$ smooth with compact support, by (2.14) and (2.15),

$$
\begin{aligned}
& \sum_{t_{j}}\left(e^{-\left(\frac{t_{j}-T}{T^{1-\epsilon}}\right)^{2}}+e^{-\left(\frac{t_{j}+T}{T^{1-\epsilon}}\right)^{2}}\right) L\left(1, \operatorname{sym}^{2} \varphi_{j}\right)\left\langle P_{\varphi_{1}, m_{1}},\left|\mu_{j}\right|^{2}\right\rangle \overline{\left\langle P_{\varphi_{2}, m_{2}},\left|\mu_{j}\right|^{2}\right\rangle} \\
& =\int_{-\infty}^{\infty} \int_{-\infty}^{\infty} \hat{\psi}_{1}\left(t_{1}\right) \hat{\psi}_{2}\left(t_{2}\right) \sum_{t_{j}}\left(e^{-\left(\frac{t_{j}-T}{T^{1-\epsilon}}\right)^{2}}+e^{-\left(\frac{t_{j}+T}{T^{1-\epsilon}}\right)^{2}}\right) L\left(1, \operatorname{sym}^{2} \varphi_{j}\right)
\end{aligned}
$$

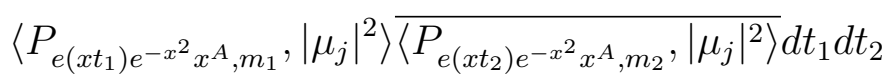




$$
\begin{aligned}
= & \int_{-\infty}^{\infty} \int_{-\infty}^{\infty} \hat{\psi}_{1}\left(t_{1}\right) \hat{\psi}_{2}\left(t_{2}\right) T^{1-\epsilon} B\left(P_{e\left(x t_{1}\right) e^{-x^{2} x^{A}, m_{1}}}, P_{e\left(x t_{2}\right) e^{-x^{2}} x^{A}, m_{2}}\right) d t_{1} d t_{2} \\
& +O\left(T^{\frac{1}{2}-\epsilon}\right) \\
= & T^{1-\epsilon} B\left(P_{\varphi_{1}, m_{1}}, P_{\varphi_{2}, m_{2}}\right)+O\left(T^{\frac{1}{2}-\epsilon}\right) .
\end{aligned}
$$

For the last equality, we applied the expression of $B$ in Proposition 2.1.

Therefore, we obtain the preliminary version for first part of Theorem 1.5:

Theorem 2.2. Let $\varphi_{j}$ denote the eigenfunctions of Laplacian, with the corresponding eigenvalues $\lambda_{j}=\frac{1}{4}+t_{j}^{2}$. For $m_{1}, m_{2} \neq 0, h_{1}, h_{2}$ be smooth functions with compact support for $i=1,2$, fix $\epsilon>0$, we have

$$
\begin{aligned}
& \sum_{t_{j}}\left(e^{-\left(\frac{t_{j}-T}{T^{1-\epsilon}}\right)^{2}}+e^{-\left(\frac{t_{j}+T}{T^{1-\epsilon}}\right)^{2}}\right) L\left(1, \operatorname{sym}^{2} \varphi_{j}\right)\left\langle P_{h_{1}, m_{1}},\left|\mu_{j}\right|^{2}\right\rangle \overline{\left\langle P_{h_{2}, m_{2}},\left|\mu_{j}\right|^{2}\right\rangle} \\
+ & \int_{0}^{\infty}\left(e^{-\left(\frac{t-T}{T^{1-\epsilon}}\right)^{2}}+e^{-\left(\frac{t+T}{T^{1-\epsilon}}\right)^{2}}\right)|\zeta(1+2 i t)|^{2}<P_{h_{1}, m_{1}},\left|\mu_{t}\right|^{2}>\overline{<P_{h_{2}, m_{2}},\left|\mu_{t}\right|^{2}>} d t \\
= & T^{1-\epsilon} B\left(P_{h_{1}, m_{1}}, P_{h_{2}, m_{2}}\right)+O\left(T^{\frac{1}{2}-\epsilon}\right),
\end{aligned}
$$

where B satisfies the expression in Proposition 2.1.

Remark 2.2. If any incomplete Poincaré series in the above theorem is replaced by incomplete Eisenstein series, i.e. $m_{i}=0$ with zero mean, the theorem is still valid. For the case $m_{1}=m_{2}=0$, there is a slight change for $B$ :

$$
\begin{aligned}
& \sum_{d_{1}, d_{2} \geq 1} \int_{0}^{\infty} \int_{0}^{1} \frac{h_{1}\left(\frac{\xi \sqrt{\tau(1-\tau)}}{d_{1}}\right)}{\tau(1-\tau)} d \tau \int_{0}^{1} \frac{h_{2}\left(\frac{\xi \sqrt{\eta(1-\eta)}}{d_{2}}\right)}{\eta(1-\eta)} d \eta \frac{d \xi}{\xi^{2}} \\
= & \int_{0}^{\infty} \int_{0}^{1} \frac{\sum_{d_{1} \geq 1} h_{1}\left(\frac{\xi \sqrt{\tau(1-\tau)}}{d_{1}}\right)}{\tau(1-\tau)} d \tau \int_{0}^{1} \frac{\sum_{d_{2} \geq 1} h_{2}\left(\frac{\xi \sqrt{\eta(1-\eta)}}{d_{2}}\right)}{\eta(1-\eta)} d \eta \frac{d \xi}{\xi^{2}}
\end{aligned}
$$

By Euler-MacLaurin summation formula, we have

$$
\sum_{d_{1} \geq 1} h_{1}\left(\frac{\xi \sqrt{\tau(1-\tau)}}{d_{1}}\right)=-\int_{0}^{\infty} b_{2}(\alpha) H_{1}\left(\frac{\xi \sqrt{\tau(1-\tau)}}{\alpha}\right) \frac{d \alpha}{\alpha^{2}}
$$


where $b_{2}(\alpha)$ is the Bernoulli polynomial of degree $2, H_{1}(x)=\left(h_{1}^{\prime}(x) x^{2}\right)^{\prime}$. For the sum over $d_{2}$, we have the similar expression. 


\section{CHAPTER 3 SYMMETRY PROPERTIES OF $B$}

In this chapter we analyze the bilinear form $B$ and using the series expression as obtained in last chapter, we prove it is self-adjoint with respect to the Laplacian $\Delta$ and the Hecke operators $T_{n}, n \geq 1$, i.e., for Poincaré series $\psi_{1}, \psi_{2}, B$ satisfies the symmetries

$$
B\left(\Delta \psi_{1}, \psi_{2}\right)=B\left(\psi_{1}, \Delta \psi_{2}\right)
$$

and for $n \geq 1$,

$$
B\left(T_{n} \psi_{1}, \psi_{2}\right)=B\left(\psi_{1}, T_{n} \psi_{2}\right)
$$

\subsection{Self-adjointness of Laplacian for $B$}

Let $L_{m}=L_{m}^{x}$ be the differential operator on $C_{0}^{\infty}(0, \infty)$ given by

$$
L_{m} h(x)=\left(x^{2} \frac{d^{2}}{d x^{2}}-4 \pi^{2} m^{2} x^{2}\right) h(x)
$$

If we define the inner product on $C_{0}^{\infty}(0, \infty)$ by

$$
\left(h_{1}, h_{2}\right)=\int_{0}^{\infty} h_{1}(x) \overline{h_{2}(x)} \frac{d x}{x^{2}}
$$

then $L_{m}$ is symmetric with respect to $($,$) , i.e.$

$$
\left(L_{m} h_{1}, h_{2}\right)=\left(h_{1}, L_{m} h_{2}\right)
$$


It is easy to check

$$
\Delta(h(y) e(m x))=\left(L_{m} h\right)(y) e(m x),
$$

hence

$$
\Delta P_{h, m}=P_{L_{m} h, m}
$$

To prove (3.1), we first deal with the case of $m_{1} m_{2} \neq 0$.

We want to show

$$
B\left(P_{L_{m_{1}} h_{1}, m_{1}}, P_{h_{2}, m_{2}}\right)=B\left(P_{h_{1}, m_{1}}, P_{L_{m_{2}} h_{2}, m_{2}}\right)
$$

For the diagonal part, it is suffice to prove,

$$
\int_{0}^{\infty} \int_{0}^{1} \frac{\cos \left(\frac{\pi m_{1}}{d_{1}} \xi(2 \tau-1)\right) L_{m_{1}} h_{1}\left(\frac{\xi \sqrt{\tau(1-\tau)}}{d_{1}}\right)}{\tau(1-\tau)} d \tau \int_{0}^{1} \frac{\cos \left(\frac{\pi m_{2}}{d_{2}} \xi(2 \eta-1)\right) h_{2}\left(\frac{\xi \sqrt{\eta(1-\eta)}}{d_{2}}\right)}{\eta(1-\eta)} d \eta \frac{d \xi}{\xi^{2}}
$$

is symmetric with respect to $h_{1}, h_{2}$.

By integration by part,

$$
\begin{aligned}
& \int_{0}^{\infty} \int_{0}^{1} \frac{\cos \left(\frac{\pi m_{1}}{d_{1}} \xi(2 \tau-1)\right) h_{1}^{\prime \prime}\left(\frac{\xi \sqrt{\tau(1-\tau)}}{d_{1}}\right)}{d_{1}^{2}} d \tau \int_{0}^{1} \frac{\cos \left(\frac{\pi m_{2}}{d_{2}} \xi(2 \eta-1)\right) h_{2}\left(\frac{\xi \sqrt{\eta(1-\eta)}}{d_{2}}\right)}{\eta(1-\eta)} d \eta d \xi \\
& =-\int_{0}^{\infty} \int_{0}^{1} \int_{0}^{1} \frac{\cos \left(\frac{\pi m_{1}}{d_{1}} \xi(2 \tau-1)\right) h_{1}^{\prime}\left(\frac{\xi \sqrt{\tau(1-\tau)}}{d_{1}}\right)}{d_{1} \sqrt{\tau(1-\tau)}} \frac{\cos \left(\frac{\pi m_{2}}{d_{2}} \xi(2 \eta-1)\right) h_{2}^{\prime}\left(\frac{\xi \sqrt{\eta(1-\eta)}}{d_{2}}\right)}{d_{2} \sqrt{\eta(1-\eta)}} d \tau d \eta d \xi \\
& +\int_{0}^{\infty} \int_{0}^{1} \int_{0}^{1} \frac{h_{1}^{\prime}\left(\frac{\xi \sqrt{\tau(1-\tau)}}{d_{1}}\right)}{d \sqrt{\tau(1-\tau)}} \frac{h_{2}\left(\frac{\xi \sqrt{\eta(1-\eta)}}{d_{2}}\right)}{\eta(1-\eta)}\left(\sin \left(\frac{\pi m_{1}}{d_{1}} \xi(2 \tau-1)\right) \cos \left(\frac{\pi m_{2}}{d_{2}} \xi(2 \eta-1)\right)\right. \\
& \left.\frac{\pi m_{1}}{d_{1}}(2 \tau-1)+\cos \left(\frac{\pi m_{1}}{d_{1}} \xi(2 \tau-1)\right) \sin \left(\frac{\pi m_{2}}{d_{2}} \xi(2 \eta-1)\right) \frac{\pi m_{2}}{d_{2}}(2 \eta-1)\right) d \tau d \eta d \xi
\end{aligned}
$$

The first integral in the above sum is symmetric with respect to $h_{1}$ and $h_{2}$. For the second integral, by integration by part in terms of $\tau$, it is easy to check the first term 
including $\sin \left(\frac{\pi m_{1}}{d_{1}} \xi(2 \tau-1)\right) \cos \left(\frac{\pi m_{2}}{d_{2}} \xi(2 \eta-1)\right)$ can be cancelled with the other term in the laplacian operator $L_{m_{1}}$,

$4 \pi^{2} \int_{0}^{\infty} \int_{0}^{1} \frac{m_{1}^{2} \cos \left(\frac{\pi m_{1}}{d_{1}} \xi(2 \tau-1)\right) h_{1}\left(\frac{\xi \sqrt{\tau(1-\tau)}}{d_{1}}\right)}{d_{1}^{2}} d \tau \int_{0}^{1} \frac{\cos \left(\frac{\pi m_{2}}{d_{2}} \xi(2 \eta-1)\right) h_{2}\left(\frac{\xi \sqrt{\eta(1-\eta)}}{d_{2}}\right)}{\eta(1-\eta)} d \eta d \xi$

For the second term including $\cos \left(\frac{\pi m_{1}}{d_{1}} \xi(2 \tau-1)\right) \sin \left(\frac{\pi m_{2}}{d_{2}} \xi(2 \eta-1)\right)$, by integration by parts in terms of $\xi$ and $\eta$, it equals

$-\pi^{2} \frac{m_{2}^{2}}{d_{2}^{2}} \int_{0}^{\infty} \int_{0}^{1} \frac{\cos \left(\frac{\pi m_{1}}{d_{1}} \xi(2 \tau-1)\right) h_{1}\left(\frac{\xi \sqrt{\tau(1-\tau)}}{d_{1}}\right)}{\tau(1-\tau)} d \tau \int_{0}^{1} \frac{\cos \left(\frac{\pi m_{2}}{d_{2}} \xi(2 \eta-1)\right) h_{2}\left(\frac{\xi \sqrt{\eta(1-\eta)}}{d_{2}}\right)}{\eta(1-\eta)} d \eta d \xi$

It is symmetric since $\frac{m_{1}}{d_{1}}=\frac{m_{2}}{d_{2}}$.

Note: In holomorphic case, the integral in the diagonal term is

$$
\int_{0}^{\infty} h_{1}\left(\frac{\xi}{d_{1}}\right) h_{2}\left(\frac{\xi}{d_{2}}\right) \frac{d \xi}{\xi^{2}}
$$

which is self-adjoint with respect to $\Delta$.

For the non-diagonal part, by change of variables

$$
\xi \rightarrow \frac{m_{2}}{d_{2}} \xi, \quad \phi \rightarrow \frac{m_{1}}{d_{1}} \phi
$$

we symmetrize the integral kernel to

$$
\frac{\sin A \cos B \cos C}{\tau \eta(1-\tau)(1-\eta)(\xi \phi)^{\frac{3}{2}}}
$$

where

$$
\begin{aligned}
& A=-\frac{\pi}{4}-\frac{\pi m_{1} m_{2} \xi}{2 c d_{1} d_{2} \phi}-\frac{\pi m_{1} m_{2} \phi}{2 c d_{1} d_{2} \xi}+2 \pi d_{1} d_{2} m_{1} m_{2} c \xi \phi \\
& B=\pi m_{1} m_{2} \xi(2 \tau-1) \quad \text { and } \quad C=\pi m_{1} m_{2} \phi(2 \eta-1) .
\end{aligned}
$$

We can verify the following symmetry

$$
\left(\xi^{2} \frac{d^{2}}{d \xi^{2}}-4 \pi^{2} m_{1}^{2} m_{2}^{2} \xi^{2} \tau(1-\tau)\right) K(\xi, \phi)=\left(\phi^{2} \frac{d^{2}}{d \phi^{2}}-4 \pi^{2} m_{1}^{2} m_{2}^{2} \phi^{2} \eta(1-\eta)\right) K(\xi, \phi),
$$


where

$$
K(\xi, \phi)=\sqrt{\xi \phi} \sin A \cos B \cos C
$$

and $A, B, C$ as above.

To show this, we check the symmetry in the following terms of

$$
\begin{aligned}
& \left(\xi^{2} \frac{d^{2}}{d \xi^{2}}-4 \pi^{2} m_{1}^{2} m_{2}^{2} \xi^{2} \tau(1-\tau)\right) K(\xi, \eta) \\
= & -\xi^{2} \sin A \cos B \cos C \sqrt{\xi \eta}\left(-\frac{\pi m_{1} m_{2}}{2 c d_{1} d_{2} \eta}+\frac{\pi m_{1} m_{2} \eta}{2 c d_{1} d_{2} \xi^{2}}+2 \pi m_{1} m_{2} d_{1} d_{2} \eta c\right)^{2} \\
& -2 \xi^{2} \cos A \sin B \cos C \sqrt{\xi \eta}\left(-\frac{\pi m_{1} m_{2}}{2 c d_{1} d_{2} \eta}+\frac{\pi m_{1} m_{2} \eta}{2 c d_{1} d_{2} \xi^{2}}+2 \pi m_{1} m_{2} d_{1} d_{2} \eta c\right) \\
& \pi m_{1} m_{2}(2 \tau-1) \\
& +\xi^{2} \cos A \cos B \cos C \sqrt{\frac{\eta}{\xi}}\left(-\frac{\pi m_{1} m_{2}}{2 c d_{1} d_{2} \eta}+\frac{\pi m_{1} m_{2} \eta}{2 c d_{1} d_{2} \xi^{2}}+2 \pi m_{1} m_{2} d_{1} d_{2} \eta c\right) \\
& +\xi^{2} \cos A \cos B \cos C \sqrt{\eta \xi}\left(-\frac{\pi m_{1} m_{2} \eta}{c d_{1} d_{2} \xi^{3}}\right) \\
& -\xi^{2} \sin A \cos B \cos C \sqrt{\eta \xi}\left(2 \pi m_{1} m_{2}(2 \tau-1)\right)^{2} \\
& -\xi^{2} \sin A \sin B \cos C \sqrt{\frac{\eta}{\xi}}\left(2 \pi m_{1} m_{2}(2 \tau-1)\right) \\
& -4 \pi^{2} m_{1}^{2} m_{2}^{2} \xi^{2} \sin A \cos B \cos C \sqrt{\eta \xi} \tau(1-\tau) \\
& -\sin A \cos B \cos C \sqrt{\xi \eta}
\end{aligned}
$$

By some careful calculation, it can be seen the above terms are symmetric with respect to $\xi$ and $\eta$, for example, the sum of (3.4), (3.8) and (3.10) can be canceled and only some symmetric terms left. More precisely, these three parts are

$$
\begin{aligned}
& -2 \pi^{2} m_{1}^{2} m_{2}^{2} \xi^{2} \sin A \cos B \cos C \sqrt{\eta \xi}-\xi^{2} \sin A \cos B \cos C \sqrt{\xi \eta} \\
& \left(-\frac{\pi m_{1} m_{2}}{2 c d_{1} d_{2} \eta}+\frac{\pi m_{1} m_{2} \eta}{2 c d_{1} d_{2} \xi^{2}}+2 \pi m_{1} m_{2} d_{1} d_{2} \eta c\right)^{2} \\
= & -2 \pi^{2} m_{1}^{2} m_{2}^{2} \xi^{2} \sin A \cos B \cos C \sqrt{\eta \xi}-\xi^{2} \sin A \cos B \cos C \sqrt{\xi \eta} \\
& \left(\left(\frac{\pi m_{1} m_{2}}{2 c d_{1} d_{2} \eta}\right)^{2}+\left(\frac{\pi m_{1} m_{2} \eta}{2 c d_{1} d_{2} \xi^{2}}\right)^{2}+\left(2 \pi m_{1} m_{2} d_{1} d_{2} \eta c\right)^{2}-\frac{\pi^{2} m_{1}^{2} m_{2}^{2}}{2 c^{2} d_{1}^{2} d_{2}^{2} \xi^{2}}\right. \\
& -2 \pi^{2} m_{1}^{2} m_{2}^{2}+\frac{2 \pi^{2} m_{1}^{2} m_{2}^{2} \eta^{2}}{\xi^{2}}
\end{aligned}
$$




$$
\begin{aligned}
= & -2 \pi^{2} m_{1}^{2} m_{2}^{2}\left(\xi^{2}+\eta^{2}\right) \sin A \cos B \cos C \sqrt{\eta \xi}-\pi^{2} m_{1}^{2} m_{2}^{2} \xi^{2} \sin A \cos B \cos C \sqrt{\xi \eta} \\
& \left(\left(\frac{1}{2 c d_{1} d_{2} \eta}\right)^{2}+\left(\frac{\eta}{2 c d_{1} d_{2} \xi^{2}}\right)^{2}+\left(2 d_{1} d_{2} \eta c\right)^{2}-\frac{1}{2 c^{2} d_{1}^{2} d_{2}^{2} \xi^{2}}\right) \\
= & -2 \pi^{2} m_{1}^{2} m_{2}^{2}\left(\xi^{2}+\eta^{2}\right) \sin A \cos B \cos C \sqrt{\eta \xi}-\pi^{2} m_{1}^{2} m_{2}^{2} \sin A \cos B \cos C \\
& \left(\left(\frac{1}{2 c d_{1} d_{2}}\right)^{2} \frac{\xi^{5 / 2}}{\eta^{3 / 2}}+\left(\frac{1}{2 c d_{1} d_{2}}\right)^{2} \frac{\eta^{3 / 2}}{\xi^{5 / 2}}+\left(2 d_{1} d_{2} c\right)^{2}(\xi \eta)^{5 / 2}-\frac{1}{2 c^{2} d_{1}^{2} d_{2}^{2}}\right)
\end{aligned}
$$

Thus, combine with (3.3) and the expression of $B$ in Theorem 2.2, we obtain (3.1) when $m_{1} m_{2} \neq 0$.

If $m_{1} m_{2}=0$, it can be shown by using the continuity argument for the desymmetrized integral kernel.

\subsection{Self-adjointness of Hecke Operators for $B$}

In order to prove (3.2), we can check it for each Hecke operator $T_{p}$, where $p$ is a prime, i.e.

$$
B\left(T_{p} P_{h_{1}, m_{1}}, P_{h_{2}, m_{2}}\right)=B\left(P_{h_{1}, m_{1}}, T_{p} P_{h_{2}, m_{2}}\right)
$$

We will use the fact (see the proof of Theorem 6.9 in [16]

$$
T_{n} P_{h, m}(z)=\sum_{d \mid(m, n)}\left(\frac{d^{2}}{n}\right)^{\frac{1}{2}} P_{h\left(\frac{n y}{d^{2}}\right), \frac{m n}{d^{2}}}(z),
$$

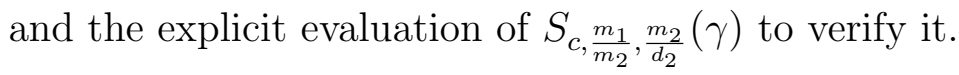

Write

$$
B\left(P_{h_{1}, m_{1}}, P_{h_{2}, m_{2}}\right)=B_{\infty}\left(P_{h_{1}, m_{1}}, P_{h_{2}, m_{2}}\right)+B_{f}\left(P_{h_{1}, m_{1}}, P_{h_{2}, m_{2}}\right),
$$

where

$$
B_{\infty}\left(P_{h_{1}, m_{1}}, P_{h_{2}, m_{2}}\right)=\sum_{\substack{d_{1}\left|m_{1}, d_{2}\right| m_{2} \\ m_{1} / d_{1}=m_{2} / d_{2}}} \int_{0}^{\infty} \int_{0}^{1} \frac{h_{1}\left(\frac{\xi \sqrt{\tau(1-\tau)}}{d_{1}}\right)}{\tau(1-\tau)} d \tau \int_{0}^{1} \frac{h_{2}\left(\frac{\xi \sqrt{\eta(1-\eta)}}{d_{2}}\right)}{\eta(1-\eta)} d \eta \frac{d \xi}{\xi^{2}}
$$


and

$$
\begin{aligned}
& B_{f}\left(P_{h_{1}, m_{1}}, P_{h_{2}, m_{2}}\right) \\
= & \sum_{\substack{d_{1}\left|m_{1} \\
d_{2}\right| m_{2}}} \sum_{c \geq 1} \operatorname{Im}\left(\frac{S_{c} \zeta_{8}}{c^{\frac{3}{2}}} \iint_{\mathrm{R}^{2}} e_{c}\left(\frac{m_{1} m_{2}}{2 d_{1} d_{2}}-\frac{m_{1}^{2} \phi}{4 d_{1}^{2} \xi}-\frac{m_{2}^{2} \xi}{4 d_{2}^{2} \phi}\right) \frac{2^{\frac{3}{2}}}{(\xi \phi)^{\frac{3}{2}}} e(\xi \phi c)\right. \\
& \left.\int_{0}^{1} \int_{0}^{1} \frac{e^{\frac{2 i \phi \eta m_{2}}{d_{2}}-\frac{2 i \xi \tau m_{1}}{d_{1}}}}{\tau \eta(1-\tau)(1-\eta)} h_{1}\left(\frac{\xi \sqrt{\tau(1-\tau)}}{2 \pi d_{1}}\right) h_{2}\left(\frac{\phi \sqrt{\eta(1-\eta)}}{2 \pi d_{2}}\right) d \tau d \eta d \xi d \phi\right)
\end{aligned}
$$

We consider the following 4 cases:

(1) If $p \nmid m_{1} m_{2}, B_{\infty}\left(T_{p} P_{h_{1}, m_{1}}, P_{h_{2}, m_{2}}\right)=B_{\infty}\left(P_{h_{1}, m_{1}}, T_{p} P_{h_{2}, m_{2}}\right)$;

(2) If $p \nmid m_{1} m_{2}, B_{f}\left(T_{p} P_{h_{1}, m_{1}}, P_{h_{2}, m_{2}}\right)=B_{f}\left(P_{h_{1}, m_{1}}, T_{p} P_{h_{2}, m_{2}}\right)$;

(3) If $p^{a} \|\left(m_{1}, m_{2}\right), B_{\infty}\left(T_{p} P_{h_{1}, m_{1}}, P_{h_{2}, m_{2}}\right)=B_{\infty}\left(P_{h_{1}, m_{1}}, T_{p} P_{h_{2}, m_{2}}\right)$;

(4) If $p^{a} \|\left(m_{1}, m_{2}\right), B_{f}\left(T_{p} P_{h_{1}, m_{1}}, P_{h_{2}, m_{2}}\right)=B_{f}\left(P_{h_{1}, m_{1}}, T_{p} P_{h_{2}, m_{2}}\right)$.

To prove (1), we use the fact

$$
T_{p} P_{h, m}(z)=p^{-\frac{1}{2}} P_{h(p \cdot), p m}(z)
$$

from (3.4). Also, from the conditions $d_{1}\left|p m_{1}, d_{2}\right| m_{2}$ and $\frac{p m_{1}}{d_{1}}=\frac{m_{2}}{d_{2}}$ we have $p \mid d_{1}$. Thus, making the change of variable $d_{1} \rightarrow p d_{1}$, we have

$$
\begin{aligned}
& B_{\infty}\left(T_{p} P_{h_{1}, m_{1}}, P_{h_{2}, m_{2}}\right) \\
= & p^{-\frac{1}{2}} B_{\infty}\left(P_{h_{1}(p \cdot), p m_{1}}, P_{h_{2}, m_{2}}\right) \\
= & p^{-\frac{1}{2}} \sum_{\substack{d_{1}\left|m_{1}, d_{2}\right| m_{2} \\
m_{1} / d_{1}=m_{2} / d_{2}}} \int_{0}^{\infty} \int_{0}^{1} \frac{h_{1}\left(\frac{\xi \sqrt{\tau(1-\tau)}}{d_{1}}\right)}{\tau(1-\tau)} d \tau \int_{0}^{1} \frac{h_{2}\left(\frac{\xi \sqrt{\eta(1-\eta)}}{d_{2}}\right)}{\eta(1-\eta)} d \eta \frac{d \xi}{\xi^{2}} \\
= & p^{-\frac{1}{2}} B_{\infty}\left(P_{h_{1}, m_{1}}, P_{h_{2}(p \cdot), p m_{2}}\right) \\
= & B_{\infty}\left(P_{h_{1}, m_{1}}, T_{p} P_{h_{2}, m_{2}}\right) .
\end{aligned}
$$

For (2), we have 


$$
\begin{aligned}
& B_{f}\left(T_{p} P_{h_{1}, m_{1}}, P_{h_{2}, m_{2}}\right) \\
= & p^{-\frac{1}{2}} B_{f}\left(P_{h_{1}(p \cdot), p m_{1}}, P_{h_{2}, m_{2}}\right) \\
= & p^{-\frac{1}{2}} \sum_{d_{1} \mid p m_{1}} \sum_{c \geq 1} \operatorname{Im}\left(\frac{S_{c} \zeta_{8}}{c^{\frac{3}{2}}} \iint_{\mathrm{R}^{2}} e_{c}\left(\frac{p m_{1} m_{2}}{2 d_{1} d_{2}}-\frac{p^{2} m_{1}^{2} \phi}{4 d_{1}^{2} \xi}-\frac{m_{2}^{2} \xi}{4 d_{2}^{2} \phi}\right) \frac{2^{\frac{3}{2}}}{(\xi \phi)^{\frac{3}{2}}} e(\xi \phi c) \int_{0}^{1} \int_{0}^{1}\right. \\
& \left.\frac{e^{\frac{2 i \phi \eta m_{2}}{d_{2}}-\frac{2 i \xi \tau p m_{1}}{d_{1}}}}{\tau \eta(1-\tau)(1-\eta)} h_{1}\left(\frac{p \xi \sqrt{\tau(1-\tau)}}{2 \pi d_{1}}\right) h_{2}\left(\frac{\phi \sqrt{\eta(1-\eta)}}{2 \pi d_{2}}\right) d \tau d \eta d \xi d \phi\right) \\
= & p^{-\frac{1}{2}} \sum_{d_{1} \mid m_{1}} \sum_{c \geq 1} \operatorname{Im}\left(\frac{S_{c} \zeta_{8}}{c^{\frac{3}{2}}} \iint_{\mathrm{R}^{2}} e_{c}\left(\frac{p m_{1} m_{2}}{2 d_{1} d_{2}}-\frac{p^{2} m_{1}^{2} \phi}{4 d_{1}^{2} \xi}-\frac{m_{2}^{2} \xi}{4 d_{2}^{2} \phi}\right) \frac{2^{\frac{3}{2}}}{(\xi \phi)^{\frac{3}{2}}} e(\xi \phi c) \int_{0}^{1} \int_{0}^{1}\right. \\
& \left.\frac{e^{\frac{2 i \phi m_{2}}{d_{2}}-\frac{2 i \xi \tau p m_{1}}{\tau d_{1}}}}{\tau \eta(1-\tau)(1-\eta)} h_{1}\left(\frac{p \xi \sqrt{\tau(1-\tau)}}{2 \pi d_{1}}\right) h_{2}\left(\frac{\phi \sqrt{\eta(1-\eta)}}{2 \pi d_{2}}\right) d \tau d \eta d \xi d \phi\right) \\
& +p^{-\frac{1}{2}} \sum_{d_{1} \mid m_{1}} \sum_{c \geq 1} \operatorname{Im}\left(\frac{S_{c} \zeta_{8}}{c^{\frac{3}{2}}} \iint_{\mathrm{R}^{2}} e_{c}\left(\frac{m_{1} m_{2}}{2 d_{1} d_{2}}-\frac{m_{1}^{2} \phi}{4 d_{1}^{2} \xi}-\frac{m_{2}^{2} \xi}{4 d_{2}^{2} \phi}\right) \frac{2^{\frac{3}{2}}}{(\xi \phi)^{\frac{3}{2}}} e(\xi \phi c) \int_{0}^{1} \int_{0}^{1}\right. \\
& \left.\frac{e^{\frac{2 i \phi \eta m_{2}}{d_{2}}-\frac{2 i \xi \tau m_{1}}{d_{1}}}}{\tau \eta(1-\tau)(1-\eta)} h_{1}\left(\frac{\xi \sqrt{\tau(1-\tau)}}{2 \pi d_{1}}\right) h_{2}\left(\frac{\phi \sqrt{\eta(1-\eta)}}{2 \pi d_{2}}\right) d \tau d \eta d \xi d \phi\right)
\end{aligned}
$$

The first sums in (3.13) and (3.14) correspond to the conditions $p \nmid d_{1}$, and $p \mid d_{1}$ respectively.

Similarly, we have

$$
\begin{aligned}
& B_{f}\left(P_{h_{1}, m_{1}}, T_{p} P_{h_{2}, m_{2}}\right) \\
= & p^{-\frac{1}{2}} \sum_{\substack{d_{1}\left|m_{1} \\
d_{2}\right| m_{2}}} \sum_{c \geq 1} \operatorname{Im}\left(\frac{S_{c} \zeta_{8}}{c^{\frac{3}{2}}} \iint_{\mathrm{R}^{2}} e_{c}\left(\frac{p m_{1} m_{2}}{2 d_{1} d_{2}}-\frac{m_{1}^{2} \phi}{4 d_{1}^{2} \xi}-\frac{p^{2} m_{2}^{2} \xi}{4 d_{2}^{2} \phi}\right) \frac{2^{\frac{3}{2}}}{(\xi \phi)^{\frac{3}{2}}} e(\xi \phi c) \int_{0}^{1} \int_{0}^{1}\right. \\
& \left.\frac{e^{\frac{2 i \phi \eta m_{2}}{d_{2}}-\frac{2 i \xi \tau m_{1}}{d_{1}}}}{\tau \eta(1-\tau)(1-\eta)} h_{1}\left(\frac{\xi \sqrt{\tau(1-\tau)}}{2 \pi d_{1}}\right) h_{2}\left(\frac{p \phi \sqrt{\eta(1-\eta)}}{2 \pi d_{2}}\right) d \tau d \eta d \xi d \phi\right) \\
& +p^{-\frac{1}{2}} \sum_{d_{1} \mid m_{1}} \sum_{c \geq 1} \operatorname{Im}\left(\frac{S_{c} \zeta_{8}}{c^{\frac{3}{2}}} \iint_{\mathrm{R}^{2}} e_{c}\left(\frac{m_{1} m_{2}}{2 d_{1} d_{2}}-\frac{m_{1}^{2} \phi}{4 d_{1}^{2} \xi}-\frac{m_{2}^{2} \xi}{4 d_{2}^{2} \phi}\right) \frac{2^{\frac{3}{2}}}{(\xi \phi)^{\frac{3}{2}}} e(\xi \phi c) \int_{0}^{1} \int_{0}^{1}\right. \\
& \left.\frac{e^{\frac{2 i \phi \eta m_{2}}{d_{2}}-\frac{2 i \xi \tau m_{1}}{d_{1}}}}{\tau \eta(1-\tau)(1-\eta)} h_{1}\left(\frac{\xi \sqrt{\tau(1-\tau)}}{2 \pi d_{1}}\right) h_{2}\left(\frac{\phi \sqrt{\eta(1-\eta)}}{2 \pi d_{2}}\right) d \tau d \eta d \xi d \phi\right)
\end{aligned}
$$


(3.14) is same as (3.16). Making the change of variables $\xi \rightarrow \frac{\xi}{p}, \phi \rightarrow p \phi$, we have (3.13) is equal to (3.15), i.e., we have proved (2).

In the cases (3) and (4), we use the fact

$$
T_{p} P_{h, m}(z)=p^{-\frac{1}{2}} P_{h(p \cdot), p m}(z)+p^{\frac{1}{2}} P_{h\left(\frac{\dot{p}}{p}\right), \frac{m}{p}}(z) .
$$

where if $p \nmid m$, we understand that $P_{h\left(\frac{\dot{p}}{p}\right), \frac{m}{p}}(z)=0$.

Thus, for the case (3), we have

$$
\begin{aligned}
& B_{\infty}\left(T_{p} P_{h_{1}, m_{1}}, P_{h_{2}, m_{2}}\right) \\
= & p^{-\frac{1}{2}} B_{\infty}\left(P_{h_{1}(p \cdot), p m_{1}}, p_{h_{2}, m_{2}}\right)+p^{\frac{1}{2}} B_{\infty}\left(P_{h_{1}(\dot{\bar{p}}), \frac{m_{1}}{p}}, P_{h_{2}, m_{2}}\right) \\
= & A+B
\end{aligned}
$$

Similarly,

$$
\begin{aligned}
& B_{\infty}\left(P_{h_{1}, m_{1}}, T_{p} P_{h_{2}, m_{2}}\right) \\
= & p^{-\frac{1}{2}} B_{\infty}\left(P_{h_{1}, m_{1}}, p_{h_{2}(p \cdot), p m_{2}}\right)+p^{\frac{1}{2}} B_{\infty}\left(P_{h_{1}, \frac{m_{1}}{p}}, P_{h_{2}(\dot{\bar{p}}), \frac{m_{2}}{p}}\right) \\
= & A_{1}+B_{1}
\end{aligned}
$$

Next, we check that

$$
\begin{gathered}
A\left(p \mid d_{1}\right)=A_{1}\left(p \mid d_{2}\right), \\
A\left(p \nmid d_{1}\right)=B_{1}\left(p \nmid d_{1}\right), \\
B\left(p \nmid d_{2}\right)=A_{1}\left(p \nmid d_{2}\right), \\
B\left(p \mid d_{2}\right)=B_{1}\left(p \mid d_{1}\right) .
\end{gathered}
$$

Hence, we get (3).

The proof of (4) is the most tedious one and we will use the induction to prove that. We have 


$$
\begin{aligned}
& B_{f}\left(T_{p} P_{h_{1}, m_{1}}, P_{h_{2}, m_{2}}\right) \\
= & p^{-\frac{1}{2}} B_{f}\left(P_{h_{1}(p \cdot), p m_{1}}, P_{h_{2}, m_{2}}\right)+p^{\frac{1}{2}} B_{f}\left(P_{h_{1}\left(\frac{\dot{p}}{p}\right), \frac{m_{1}}{p}}, P_{h_{2}, m_{2}}\right)
\end{aligned}
$$

From the expression of (2.10), it equals

$$
\begin{aligned}
& p^{-\frac{1}{2}} \sum_{\substack{d_{1}\left|p m_{1} \\
d_{2}\right| m_{2}}} \sum_{c \geq 1} \operatorname{Im}\left(\frac{S_{c} \zeta_{8}}{c^{\frac{3}{2}}} \iint_{\mathrm{R}^{2}} e_{c}\left(\frac{p m_{1} m_{2}}{2 d_{1} d_{2}}-\frac{p^{2} m_{1}^{2} \phi}{4 d_{1}^{2} \xi}-\frac{m_{2}^{2} \xi}{4 d_{2}^{2} \phi}\right) \frac{2^{\frac{3}{2}}}{(\xi \phi)^{\frac{3}{2}}} e(\xi \phi c) \int_{0}^{1} \int_{0}^{1}\right. \\
& \left.\frac{e^{\frac{2 i \phi \eta m_{2}}{d_{2}}-\frac{2 i \xi \tau p m_{1}}{d_{1}}}}{\tau \eta(1-\tau)(1-\eta)} h_{1}\left(\frac{p \xi \sqrt{\tau(1-\tau)}}{2 \pi d_{1}}\right) h_{2}\left(\frac{\phi \sqrt{\eta(1-\eta)}}{2 \pi d_{2}}\right) d \tau d \eta d \xi d \phi\right) \\
& +p^{\frac{1}{2}} \sum_{\substack{d_{1}\left|p m_{1} \\
d_{2}\right| m_{2}}} \sum_{c \geq 1} \operatorname{Im}\left(\frac{S_{c} \zeta_{8}}{c^{\frac{3}{2}}} \iint_{\mathrm{R}^{2}} e_{c}\left(\frac{p m_{1} m_{2}}{2 d_{1} d_{2}}-\frac{p^{2} m_{1}^{2} \phi}{4 d_{1}^{2} \xi}-\frac{m_{2}^{2} \xi}{4 d_{2}^{2} \phi}\right) \frac{2^{\frac{3}{2}}}{(\xi \phi)^{\frac{3}{2}}} e(\xi \phi c) \int_{0}^{1} \int_{0}^{1}\right. \\
& \left.\frac{e^{\frac{2 i \phi \eta m_{2}}{d_{2}}-\frac{2 i \xi \tau p m_{1}}{d_{1}}}}{\tau \eta(1-\tau)(1-\eta)} h_{1}\left(\frac{p \xi \sqrt{\tau(1-\tau)}}{2 \pi d_{1}}\right) h_{2}\left(\frac{\phi \sqrt{\eta(1-\eta)}}{2 \pi d_{2}}\right) d \tau d \eta d \xi d \phi\right) \\
& =p^{-\frac{1}{2}} \sum_{\substack{d_{1}\left|m_{1} \\
d_{2}\right| m_{2}}} \sum_{c \geq 1} \operatorname{Im}\left(\frac{S_{c} \zeta_{8}}{c^{\frac{3}{2}}} \iint_{\mathrm{R}^{2}} e_{c}\left(\frac{p m_{1} m_{2}}{2 d_{1} d_{2}}-\frac{p^{2} m_{1}^{2} \phi}{4 d_{1}^{2} \xi}-\frac{m_{2}^{2} \xi}{4 d_{2}^{2} \phi}\right) \frac{2^{\frac{3}{2}}}{(\xi \phi)^{\frac{3}{2}}} e(\xi \phi c) \int_{0}^{1} \int_{0}^{1}\right. \\
& \left.\frac{e^{\frac{2 i \phi \eta m_{2}}{d_{2}}-\frac{2 i \xi \tau p m_{1}}{d_{1}}}}{\tau \eta(1-\tau)(1-\eta)} h_{1}\left(\frac{p \xi \sqrt{\tau(1-\tau)}}{2 \pi d_{1}}\right) h_{2}\left(\frac{\phi \sqrt{\eta(1-\eta)}}{2 \pi d_{2}}\right) d \tau d \eta d \xi d \phi\right) \\
& +p^{\frac{1}{2}} \sum_{\substack{d_{1}\left|m_{1} \\
d_{2}\right| m_{2}}} \sum_{c \geq 1} \operatorname{Im}\left(\frac{S_{c} \zeta_{8}}{c^{\frac{3}{2}}} \iint_{\mathrm{R}^{2}} e_{c}\left(\frac{m_{1} m_{2}}{2 d_{1} d_{2}}-\frac{m_{1}^{2} \phi}{4 d_{1}^{2} \xi}-\frac{m_{2}^{2} \xi}{4 d_{2}^{2} \phi}\right) \frac{2^{\frac{3}{2}}}{(\xi \phi)^{\frac{3}{2}}} e(\xi \phi c) \int_{0}^{1} \int_{0}^{1}\right. \\
& \left.\frac{e^{\frac{2 i \phi \eta m_{2}}{d_{2}}-\frac{2 i \xi \tau m_{1}}{d_{1}}}}{\tau \eta(1-\tau)(1-\eta)} h_{1}\left(\frac{\xi \sqrt{\tau(1-\tau)}}{2 \pi d_{1}}\right) h_{2}\left(\frac{\phi \sqrt{\eta(1-\eta)}}{2 \pi d_{2}}\right) d \tau d \eta d \xi d \phi\right)
\end{aligned}
$$

We denote the above sum as $I_{1}+I_{2}$. Similarly,

$$
\begin{aligned}
& B_{f}\left(P_{h_{1}, m_{1}}, T_{p} P_{h_{2}, m_{2}}\right) \\
= & p^{-\frac{1}{2}} B_{f}\left(P_{h_{1}, m_{1}}, P_{h_{2}(p \cdot), p m_{2}}\right)+p^{\frac{1}{2}} B_{f}\left(P_{h_{1}, m_{1}}, P_{h_{2}\left(\frac{\dot{\bar{p}}}{p}, \frac{m_{2}}{p}\right)}\right.
\end{aligned}
$$

From the expression of (2.10), it equals 


$$
\begin{aligned}
& p^{-\frac{1}{2}} \sum_{\substack{d_{1}\left|p m_{1} \\
d_{2}\right| m_{2}}} \sum_{c \geq 1} \operatorname{Im}\left(\frac{S_{c} \zeta_{8}}{c^{\frac{3}{2}}} \iint_{\mathrm{R}^{2}} e_{c}\left(\frac{p m_{1} m_{2}}{2 d_{1} d_{2}}-\frac{p^{2} m_{1}^{2} \phi}{4 d_{1}^{2} \xi}-\frac{m_{2}^{2} \xi}{4 d_{2}^{2} \phi}\right) \frac{2^{\frac{3}{2}}}{(\xi \phi)^{\frac{3}{2}}} e(\xi \phi c) \int_{0}^{1} \int_{0}^{1}\right. \\
& \left.\frac{e^{\frac{2 i \phi \eta m_{2}}{d_{2}}-\frac{2 i \xi \tau p m_{1}}{d_{1}}}}{\tau \eta(1-\tau)(1-\eta)} h_{1}\left(\frac{p \xi \sqrt{\tau(1-\tau)}}{2 \pi d_{1}}\right) h_{2}\left(\frac{\phi \sqrt{\eta(1-\eta)}}{2 \pi d_{2}}\right) d \tau d \eta d \xi d \phi\right) \\
& +p^{\frac{1}{2}} \sum_{\substack{d_{1}\left|p m_{1} \\
d_{2}\right| m_{2}}} \sum_{c \geq 1} \operatorname{Im}\left(\frac{S_{c} \zeta_{8}}{c^{\frac{3}{2}}} \iint_{\mathrm{R}^{2}} e_{c}\left(\frac{p m_{1} m_{2}}{2 d_{1} d_{2}}-\frac{p^{2} m_{1}^{2} \phi}{4 d_{1}^{2} \xi}-\frac{m_{2}^{2} \xi}{4 d_{2}^{2} \phi}\right) \frac{2^{\frac{3}{2}}}{(\xi \phi)^{\frac{3}{2}}} e(\xi \phi c) \int_{0}^{1} \int_{0}^{1}\right. \\
& \left.\frac{e^{\frac{2 i \phi \eta m_{2}}{d_{2}}-\frac{2 i \xi \tau p m_{1}}{d_{1}}}}{\tau \eta(1-\tau)(1-\eta)} h_{1}\left(\frac{p \xi \sqrt{\tau(1-\tau)}}{2 \pi d_{1}}\right) h_{2}\left(\frac{\phi \sqrt{\eta(1-\eta)}}{2 \pi d_{2}}\right) d \tau d \eta d \xi d \phi\right) \\
& =p^{-\frac{1}{2}} \sum_{\substack{d_{1}\left|m_{1} \\
d_{2}\right| m_{2}}} \sum_{c \geq 1} \operatorname{Im}\left(\frac{S_{c} \zeta_{8}}{c^{\frac{3}{2}}} \iint_{\mathrm{R}^{2}} e_{c}\left(\frac{p m_{1} m_{2}}{2 d_{1} d_{2}}-\frac{p^{2} m_{1}^{2} \phi}{4 d_{1}^{2} \xi}-\frac{m_{2}^{2} \xi}{4 d_{2}^{2} \phi}\right) \frac{2^{\frac{3}{2}}}{(\xi \phi)^{\frac{3}{2}}} e(\xi \phi c) \int_{0}^{1} \int_{0}^{1}\right. \\
& \left.\frac{e^{\frac{2 i \phi \eta m_{2}}{d_{2}}-\frac{2 i \xi \tau p m_{1}}{d_{1}}}}{\tau \eta(1-\tau)(1-\eta)} h_{1}\left(\frac{p \xi \sqrt{\tau(1-\tau)}}{2 \pi d_{1}}\right) h_{2}\left(\frac{\phi \sqrt{\eta(1-\eta)}}{2 \pi d_{2}}\right) d \tau d \eta d \xi d \phi\right) \\
& +p^{\frac{1}{2}} \sum_{\substack{d_{1}\left|m_{1} \\
d_{2}\right| m_{2}}} \sum_{c \geq 1} \operatorname{Im}\left(\frac{S_{c} \zeta_{8}}{c^{\frac{3}{2}}} \iint_{\mathrm{R}^{2}} e_{c}\left(\frac{m_{1} m_{2}}{2 d_{1} d_{2}}-\frac{m_{1}^{2} \phi}{4 d_{1}^{2} \xi}-\frac{m_{2}^{2} \xi}{4 d_{2}^{2} \phi}\right) \frac{2^{\frac{3}{2}}}{(\xi \phi)^{\frac{3}{2}}} e(\xi \phi c) \int_{0}^{1} \int_{0}^{1}\right. \\
& \left.\frac{e^{\frac{2 i \phi \eta m_{2}}{d_{2}}-\frac{2 i \xi \tau m_{1}}{d_{1}}}}{\tau \eta(1-\tau)(1-\eta)} h_{1}\left(\frac{\xi \sqrt{\tau(1-\tau)}}{2 \pi d_{1}}\right) h_{2}\left(\frac{\phi \sqrt{\eta(1-\eta)}}{2 \pi d_{2}}\right) d \tau d \eta d \xi d \phi\right) \\
& =I I_{1}+I I_{2}
\end{aligned}
$$

According to whether or not $p \mid(c, *, *)$ in $S_{c, *, *}$, we can decompose the above sums $I_{1}, I_{2}, I I_{1}, I I_{2}$ into the following 8 sums

$$
I_{1}=I_{11}+I_{12}, \quad I_{2}=I_{21}+I_{22}, \quad I I_{1}=I I_{11}+I I_{12}, \quad I I_{2}=I I_{21}+I I_{22} .
$$

Note if $p \mid(c, *, *), S_{c, *, *}=0$ unless $p^{2} \mid c$. Let $c=p^{2} c_{1}$, we have

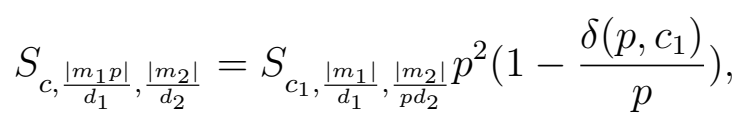

where $\delta\left(p, c_{1}\right)=0$ if $p \mid c_{1} ; \delta\left(p, c_{1}\right)=1$ if $p \nmid c_{1}$. Hence we can write $I_{11}=I_{11}^{\prime}-I_{11}^{\prime \prime}$ correspondingly. 
Similarly we have

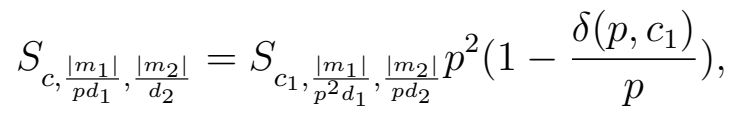

and write $I_{21}=I_{21}^{\prime}-I_{21}^{\prime \prime}$,

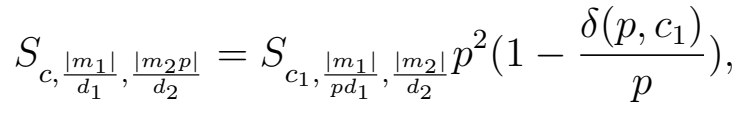

and write $I I_{11}=I I_{11}^{\prime}-I I_{11}^{\prime \prime}$,

$$
S_{c, \frac{\left|m_{1}\right|}{d_{1}}, \frac{\left|m_{2}\right|}{p d_{2}}}=S_{c_{1}, \frac{\left|m_{1}\right|}{p d_{1}}, \frac{\left|m_{2}\right|}{p^{2} d_{2}}} p^{2}\left(1-\frac{\delta\left(p, c_{1}\right)}{p}\right),
$$

and write $I I_{21}=I I_{21}^{\prime}-I I_{21}^{\prime \prime}$ corresponding $p \mid c_{1}$ or not.

By the induction hypothesis on $\left(\frac{m_{1}}{p}, \frac{m_{2}}{p}\right)$, we have $I_{11}^{\prime}+I_{21}^{\prime}=I I_{11}^{\prime}+I I_{21}^{\prime}$.

We have $S_{c p, a, b}=p^{2} S_{c, a, b}$ and $S_{t p^{2}, a p, b}=0$ if $p \nmid b c$. Using this and the evaluation of $S_{c, a, b}$ we can verify that

$$
I_{12}\left(p \mid d_{1}\right)=I I_{12}\left(p \mid d_{2}\right),
$$

where $I_{12}\left(p \mid d_{1}\right)$ means the partial sum of $I_{12}$ in which $\left.p \mid d_{1}\right)$. Similarly, we have

$$
\begin{gathered}
I_{12}\left(p \nmid d_{1}, p \nmid d_{2}, p \nmid c\right)=I I_{12}\left(p \nmid d_{2}, p \nmid d_{1}, p \nmid c\right), \\
I_{12}\left(p \nmid d_{1}, p \| d_{2}, p \nmid c\right)=I I_{12}\left(p \nmid d_{2}, p \| d_{1}, p \nmid c\right), \\
I_{12}\left(p \nmid d_{1}, p^{2} \mid d_{2}, p \nmid c\right)=I_{11}^{\prime \prime}\left(p \nmid d_{1}, p^{2} \mid m_{2} / d_{1}\right), \\
I_{12}\left(p \nmid d_{1}, p^{2} \mid d_{2}, p \nmid c\right)=I_{11}^{\prime \prime}\left(p \nmid d_{1}, p \| m_{2} / d_{2}\right), \\
I I_{11}^{\prime \prime}\left(p \nmid d_{2}, p^{2} \mid m_{1} / d_{1}\right)=I I_{12}\left(p \nmid d_{2}, p^{2} \mid d_{1}, p \nmid c\right), \\
I I_{11}^{\prime \prime}\left(p \nmid d_{2}, p \| m_{1} / d_{1}\right)=I I_{12}\left(p \nmid d_{2}, p \nmid c\right), \\
I_{11}^{\prime \prime}\left(p \mid d_{1}\right)=I I_{11}^{\prime \prime}\left(p \mid d_{2}\right), \\
I_{22}\left(p \mid d_{2}\right)=I I_{22}\left(p \mid d_{1}\right), \\
I_{22}\left(p \nmid d_{2}, p \nmid d_{1}, p \nmid c\right)=I I_{22}\left(p \nmid d_{1}, p \nmid d_{2}, p \nmid c\right),
\end{gathered}
$$




$$
\begin{gathered}
I_{22}\left(p \nmid d_{2}, p \| d_{1}, p \nmid c\right)=I I_{22}\left(p \nmid d_{1}, p \| d_{2}, p \nmid c\right), \\
I_{22}\left(p \nmid d_{2}, p^{2} \mid d_{1}, p \nmid c\right)=I_{21}^{\prime \prime}\left(p \nmid d_{2}, p^{3} \mid m_{1} / d_{1}\right), \\
I_{22}\left(p \nmid d_{2}, p \mid c\right)=I_{21}^{\prime \prime}\left(p \nmid d_{2}, p^{2} \| m_{1} / d_{1}\right), \\
I I_{21}^{\prime \prime}\left(p \mid d_{1}\right)=I_{21}^{\prime \prime}\left(p \mid d_{2}\right), \\
I I_{22}\left(p \nmid d_{1}, p^{2} \mid d_{2}, p \nmid c\right)=I I_{21}^{\prime \prime}\left(p \nmid d_{1}, p^{3} \mid m_{2} / d_{2}\right), \\
I I_{22}\left(p \nmid d_{1}, p \mid c\right)=I I_{21}^{\prime \prime}\left(p \nmid d_{1}, p^{2} \| m_{2} / d_{2}\right) .
\end{gathered}
$$

Hence we deduce from the above identities that

$$
B_{f}\left(T_{p} P_{h_{1}, m_{1}}, P_{h_{2}, m_{2}}\right)=B_{f}\left(P_{h_{1}, m_{1}}, T_{p} P_{h_{2}, m_{2}}\right) .
$$

This completes the proof of

$$
B\left(T_{p} P_{h_{1}, m_{1}}, P_{h_{2}, m_{2}}\right)=B\left(P_{h_{1}, m_{1}}, T_{p} P_{h_{2}, m_{2}}\right)
$$

for each $T_{p}, p$ is a prime. Thus, the bilinear form $B(\cdot, \cdot)$ defined on the space spanned by $P_{h, m}$ 's is self-adjoint with respect to the Hecke operators $T_{n}, n \geq 1$. 


\section{CHAPTER 4}

\section{EXTENSION AND DIAGONALIZATION OF $B$}

In this chapter, we apply the method in [34] to extend $B$ to $C_{0,0}^{\infty}(X)$, where $X=\Gamma \backslash \mathbb{H}$. If we restrict $B$ to the Maass-Hecke cusp forms, it can be shown that $B$ is diagonalized by the orthonormal basis of Hecke-Maass cusp forms. The idea of the extension is choose a partition of unity subordinate to a covering of $X$, then any $\psi \in C_{0,0}^{\infty}(X)$ can be written as a linear combination of $P_{h, m}$, where $h$ is smooth with compact support functions. From last chapter's symmetry properties of $B$, it is easy to see that $B$ can be diagonalized by the orthonormal basis of the subspace of Hecke-Maass cusp forms.

Let $P$ be the projection map from $\mathbb{H}$ to $X$, and construct the following open sets with compact closures in $\mathbb{H}$ whose projections to $X$ form a locally finite open covering of $X: D_{00}$ is a neighborhood of $i$ and the restriction of $P$ on $D_{00}$ is two to one. $D_{01}$ is a neighborhood of $e^{\frac{2 \pi i}{3}}$ and the restriction of $P$ on $D_{01}$ is three to one.

$$
\begin{gathered}
D_{02}=\left\{z|\mathfrak{I}(z)<2,| \mathfrak{R}(z)\left|<\frac{1}{2},\right| z \mid>1\right\} . \\
D_{03}=\left\{z\left|\mathfrak{I}(z)<2,-\frac{1}{2} \leq\right| \mathfrak{R}(z)|<0,| z \mid>1\right\} \cup\left\{z|\Im(z)<2,-1<| \mathfrak{I}(z)\left|\leq-\frac{1}{2},\right| z+1 \mid>1\right\} .
\end{gathered}
$$

For $k \geq 1$, let

$$
\begin{gathered}
D_{k 1}=\left\{z\left|\frac{3^{k}}{2}<\Im(z)<3^{k+1},-1<\right| \mathfrak{R}(z) \mid<0\right\} . \\
D_{k 2}=\left\{z\left|\frac{3^{k}}{2}<\Im(z)<3^{k+1},-\frac{1}{2}<\right| \mathfrak{R}(z) \mid<\frac{1}{2}\right\} .
\end{gathered}
$$


Let $\left\{f_{k j}\right\}$ be the partition of unity subordinate to the above covering of $X$, then each $f_{k j}$ can be viewed as an automorphic function with respect to $\Gamma$. We can extend $f_{k, j}$ to a smooth $\Gamma_{\infty}$ periodic function $\tilde{f}_{k, j}$ on $\mathbb{H}$. There exists $y_{0}>0$ such that $\tilde{f}_{k, j}$ are all supported in the half-plane $y \geq y_{0}$, where $\tilde{f}_{k, j}(z)=f_{k, j}(z)$, except when $k=0$ and $j=2,3$.

Let $\psi \in C_{0,0}^{\infty}(X)$, we have

$$
\psi(z)=\sum_{k, j} f_{k, j}(z) \psi(z)
$$

and

$$
f_{k, j}(z) \psi(z)=\frac{1}{n_{k, j}} \sum_{\gamma \in \Gamma_{\infty} \backslash \Gamma} \tilde{f}_{k, j}(\gamma z) \psi(\gamma z),
$$

where $n_{k, j}=2$ if $k=0$, and $j=0 ; n_{k, j}=3$ if $k=0$, and $j=1 ; n_{k, j}=1$, if otherwise.

Expanding $\tilde{f}_{k, j}(z) \psi(z)$ into the Fourier series in $x$, we have

$$
\tilde{f}_{k, j}(z) \psi(z)=\sum_{m \in \mathbf{Z}} h_{k, j, m}(y) e(m x)
$$

where $h_{k, j, m}(y)$ are smooth with compact support and satisfy the rapid decay condition

$$
h_{k, j, m}(y) \ll_{A} y^{-A}|m|^{-A}
$$

for any $A>0$. Thus, we have the following decomposition:

$$
\begin{aligned}
\psi(z) & =\sum_{k, j} \sum_{m \in \mathbf{Z}} \frac{1}{n_{k, j}} P_{h_{k, j, m}, m}(z) \\
& =\sum_{k, j} \sum_{m \neq 0} \frac{1}{n_{k, j}} P_{h_{k, j, m}, m}(z)+\sum_{k, j} P_{h_{k, j, 0}, 0}(z)-\frac{1}{2} P_{h_{0,0}, 0}(z)-\frac{2}{3} P_{h_{0,1,0}, 0}(z) \\
& =\sum_{k, j} \sum_{m \neq 0} \frac{1}{n_{k, j}} P_{h_{k, j, m}, m}(z)+P_{H, 0}(z)-\frac{1}{2} P_{h_{0,0,0}, 0}(z)-\frac{2}{3} P_{h_{0,1,0}, 0}(z),
\end{aligned}
$$

where $H(y) \in C_{c}^{\infty}(0, \infty)$ is defined as

$$
H(y)=\sum_{k, j} h_{k, j, 0}(y) .
$$


This follows from the fact that $\sum_{k, j} f_{k, j}(z)=1$, and $\tilde{f}_{k, j}(z)$ are all supported in the half-plane $y \geq y_{0}$ where we have

$$
\begin{aligned}
\sum_{k, j} h_{k, j, 0}(y) & =\int_{0}^{1}\left(\sum_{k, j} \tilde{f}_{k, j}(z)\right) \psi(z) d x \\
& =\int_{0}^{1}\left(\sum_{k, j} f_{k, j}(z)+\left(\tilde{f}_{0,2}(z)-f_{0,2}(z)\right)+\left(\tilde{f}_{0,3}(z)-f_{0,3}(z)\right)\right) \psi(z) d x \\
& =\int_{0}^{1}\left(\left(\tilde{f}_{0,2}(z)-f_{0,2}(z)\right)+\left(\tilde{f}_{0,3}(z)-f_{0,3}(z)\right)\right) \psi(z) d x
\end{aligned}
$$

since $\int_{0}^{1} \psi(z) d x=0$. Moreover we have

$$
\int_{X}\left(P_{H, 0}(z)-\frac{1}{2} P_{h_{0,0,0}, 0}(z)-\frac{2}{3} P_{h_{0,1,0}, 0}(z)\right) d \mu=0 .
$$

Let

$$
P_{H, 0}(z)-\frac{1}{2} P_{h_{0,0,0}, 0}(z)-\frac{2}{3} P_{h_{0,1,0}, 0}(z)=P_{h, 0}(z)
$$

with

$$
h=H-\frac{1}{2} h_{0,0,0}-\frac{2}{3} h_{0,1,0} .
$$

We have

$$
\psi(z)=\sum_{k, j} \sum_{m \neq 0} \frac{1}{n_{k, j}} P_{h_{k, j, m}, m}(z)+P_{h, 0}(z),
$$

with

$$
\int_{X} P_{h, 0}(z) d \mu=0
$$

Thus, it follows from Theorem 2.2 that for $\psi$ and $\phi$ in $C_{0,0}^{\infty}(X)$, we have

$$
\begin{aligned}
& \sum_{t_{j}}\left(e^{-\left(\frac{t_{j}-T}{T^{1-\epsilon}}\right)^{2}}+e^{-\left(\frac{t_{j}+T}{T^{1-\epsilon}}\right)^{2}}\right) L\left(1, \operatorname{sym}^{2} \varphi_{j}\right)\left\langle\psi,\left|\mu_{j}\right|^{2}\right\rangle \overline{\left\langle\phi,\left|\mu_{j}\right|^{2}\right\rangle} \\
+ & \int_{0}^{\infty}\left(e^{-\left(\frac{t-T}{T^{1-\epsilon}}\right)^{2}}+e^{-\left(\frac{t+T}{T^{1-\epsilon}}\right)^{2}}\right)|\zeta(1+2 i t)|^{2}<\psi,\left|\mu_{t}\right|^{2}>\overline{<\phi,\left|\mu_{t}\right|^{2}>} d t \\
= & T^{1-\epsilon} B(\psi, \phi)+O\left(T^{\frac{1}{2}-\epsilon}\right),
\end{aligned}
$$


where

$$
\begin{aligned}
B(\psi, \phi)= & \sum_{k_{1}, j_{1}, m_{1} \neq 0 ; k_{2}, j_{2}, m_{2} \neq 0} \frac{1}{n_{k_{1}, j_{1}} n_{k_{2}, j_{2}}} B\left(P_{h_{k_{1}, j_{1}, m_{1}}, m_{1}}, P_{h_{k_{2}, j_{2}, m_{2}}, m_{2}}\right) \\
& +\sum_{k_{1}, j_{1}, m_{1} \neq 0} \frac{1}{n_{k_{1}, j_{1}}} B\left(P_{h_{k_{1}, j_{1}, m_{1}}, m_{1}}, P_{h_{\phi}, 0}\right) \\
& +\sum_{k_{2}, j_{2}, m_{2} \neq 0} \frac{1}{n_{k_{2}, j_{2}}} B\left(P_{h_{\psi}, 0}, P_{h_{k_{2}, j_{2}, m_{2}}, m_{2}}\right) \\
& +B\left(P_{h_{\psi}, 0}, P_{h_{\phi}, 0}\right) .
\end{aligned}
$$

Since for $\phi$ and $\psi$ we have the rapid decay condition

$$
h_{k, j, m}(y) \ll_{A} y^{-A}|m|^{-A}
$$

for any $A>0$, we can see the above series converges abosolutely.

From last chapter's results of (3.1) and (3.2) it follows that the bilinear form $B(\psi, \phi)$ is defined on $C_{0,0}^{\infty}(X) \times C_{0,0}^{\infty}(X)$ and satisfies

$$
B(\Delta \psi, \phi)=B(\psi, \Delta \phi)
$$

and for $n \geq 1$,

$$
B\left(T_{n} \psi, \phi\right)=B\left(\psi, T_{n} \phi\right)
$$

If we restrict $B$ to the Maass-Hecke cusp forms, we can show the continuous spectrum integral is small enough, see Section 5.2.3. It can be shown that the continuous spectrum integral contributes $O\left(T^{\frac{1}{2}+\epsilon}\right)$. Hence, we obtain the first part of main Theorem 1.4.

Now restrict $B$ to the Maass-Hecke cusp forms, it is easy to see $B$ is diagonalized by the orthonormal basis of this subspace from the symmetry properties of $B$. If $\psi$, $\phi$ are two distinct Maass-Hecke cusp forms, then for $n \geq 1$,

$$
B\left(T_{n} \psi, \phi\right)=B\left(\psi, T_{n} \phi\right)
$$


We have

$$
\lambda_{n}(\psi) B(\psi, \phi)=\lambda_{n}(\phi) B(\psi, \phi)
$$

Since there must be an $n$ such that $\lambda_{n}(\psi) \neq \lambda_{n}(\phi)$ for $\psi$ and $\phi$, we obtain

$$
B(\psi, \phi)=0
$$

for distinct Maass-Hecke cusp forms. Thus, we have shown that $B$ is diagonalized by the orthonormal basis of the subspace of the Maass-Hecke cusp forms.

We are going to calculate the eigenvalue of $B$ on such a Maass-Hecke cusp form in next chapter. 


\section{CHAPTER 5 EIGENVALUES OF $B$}

In this chapter, we compute the value of $B$ on $\phi(z)$, an even Maass-Hecke cuspidal

eigenform for the modular group $\Gamma$, with the Laplacian eigenvalue $\lambda_{\phi}=\frac{1}{4}+t_{\phi}^{2}$. To do so we return to the original asymptotic of

$$
\sum_{j \geq 1}\left(e^{-\left(\frac{t_{j}-T}{T^{1-\epsilon}}\right)^{2}}+e^{-\left(\frac{t_{j}+T}{T^{1-\epsilon}}\right)^{2}}\right) L\left(1, \operatorname{sym}^{2} \phi_{j}\right)\left|\left\langle\mu_{\phi_{j}}, \phi\right\rangle\right|^{2} .
$$

After applying Watson's formula on $\left|\left\langle\mu_{\phi_{j}}, \phi\right\rangle\right|^{2}$, the quantum variance sum over $\phi_{j}$ boils down to averaging $L\left(1 / 2, \phi \otimes \operatorname{sym}^{2}\left(\phi_{j}\right)\right)$. By Rankin-Selberg theory, we can express this sum in a suitable series to which the Kuznetsov formula is applied. The estimation of diagonal and non-diagonal terms is similar to the way we did before. However, for the continuous spectrum part, we apply Jutila's subconvexity bound to show it is negligible in this case.

\subsection{Watson's Formula}

Let $L(s, \phi)$ be the associated standard $L$-function, which admits analytic continuation to the whole complex plane and satisfies the functional equation:

$$
\Lambda_{\phi}(s):=\pi^{-s} \Gamma\left(\frac{s+i t_{\phi}}{2}\right) \Gamma\left(\frac{s-i t_{\phi}}{2}\right) L(s, \phi)=\Lambda_{\phi}(1-s) .
$$

Moreover, we have

$$
\Lambda_{\mathrm{sym}^{2}(\phi)}(s)=\pi^{-3 s / 2} \Gamma\left(\frac{s}{2}\right) \Gamma\left(\frac{s}{2}+i t_{\phi}\right) \Gamma\left(\frac{s}{2}-i t_{\phi}\right) L\left(s, \operatorname{sym}^{2}(\phi)\right),
$$




$$
\begin{aligned}
\Lambda_{\psi \otimes \mathrm{sym}^{2}(\phi)}(s)= & \pi^{-3 s} \Gamma\left(\frac{s+i t_{\psi}}{2}+i t_{\phi}\right) \Gamma\left(\frac{s+i t_{\psi}}{2}-i t_{\phi}\right) \Gamma\left(\frac{s+i t_{\psi}}{2}\right) \\
& \Gamma\left(\frac{s-i t_{\psi}}{2}+i t_{\phi}\right) \Gamma\left(\frac{s-i t_{\psi}}{2}-i t_{\phi}\right) \Gamma\left(\frac{s-i t_{\psi}}{2}\right) L\left(s, \psi \otimes \operatorname{sym}^{2}(\phi)\right)
\end{aligned}
$$

here $\psi(z)$ is also a Maass-Hecke cuspidal eigenform and we normalize $\psi(z)$ so that its first Fourier coefficient $a_{\psi}(1)=1$.

By Watson's formula [49] and Stirling formua, we have

$$
\begin{aligned}
\left|\left\langle\mu_{\phi}, \psi\right\rangle\right|^{2} & =\frac{\Lambda_{\psi \otimes \mathrm{sym}^{2}(\phi)}\left(\frac{1}{2}\right) \Lambda_{\psi}\left(\frac{1}{2}\right)}{\Lambda_{\mathrm{sym}^{2}(\phi)}(1)^{2} \Lambda_{\mathrm{sym}^{2}(\psi)}(1)} \\
& =\frac{L\left(\frac{1}{2}, \psi\right) L\left(\frac{1}{2}, \psi \otimes \operatorname{sym}^{2}(\phi)\right) \cosh \left(\pi t_{\psi}\right)\left|\Gamma\left(\frac{1}{4}+\frac{i t_{\psi}}{2}\right)\right|^{4}\left|a_{\phi}(1)\right|^{2}}{t_{\phi} L\left(1, \operatorname{sym}^{2}(\phi)\right) L\left(1, \operatorname{sym}^{2}(\psi)\right)}\left(1+O\left(t_{\phi}^{-1}\right)\right) \\
& =\frac{L\left(\frac{1}{2}, \psi \otimes \phi \otimes \phi\right)\left|\Gamma\left(\frac{1}{4}+\frac{i t_{\psi}}{2}\right)\right|^{4}\left|a_{\phi}(1)\right|^{2}}{t_{\phi} L\left(1, \operatorname{sym}^{2}(\phi)\right)}\left(1+O\left(t_{\phi}^{-1}\right)\right)
\end{aligned}
$$

\subsection{The Kuznetsov Formula}

Next, we compute

$$
\sum_{j \geq 1}\left(e^{-\left(\frac{t_{j}-T}{T^{1-\epsilon}}\right)^{2}}+e^{-\left(\frac{t_{j}+T}{T^{1-\epsilon}}\right)^{2}}\right) L\left(1, \operatorname{sym}^{2} \phi_{j}\right)\left|\left\langle\mu_{\phi_{j}}, \psi\right\rangle\right|^{2}
$$

Let $\Phi$ be the cuspidal automorphic form on $G L(3)$ which is the Gelbart-Jacquet lift of the cusp form $\phi$, with the Fourier coefficients $a_{\Phi}\left(m_{1}, m_{2}\right)$ [2], where

$$
a_{\Phi}\left(m_{1}, m_{2}\right)=\sum_{d \mid\left(m_{1}, m_{2}\right)} \lambda_{\Phi}\left(\frac{m_{1}}{d}, 1\right) \lambda_{\Phi}\left(\frac{m_{2}}{d}, 1\right) \mu(d)
$$

and

$$
\lambda_{\Phi}(r, 1)=\sum_{s^{2} t=r} \lambda_{\phi}\left(t^{2}\right)
$$

The Rankin-Selberg convolution $L\left(s, \psi \otimes \operatorname{sym}^{2}(\phi)\right)$ is represented by the Dirichlet series [3],

$$
L\left(s, \psi \otimes \operatorname{sym}^{2}(\phi)\right)=\sum_{m_{1}, m_{2} \geq 1} \lambda_{\psi}\left(m_{1}\right) a_{\Phi}\left(m_{1}, m_{2}\right)\left(m_{1} m_{2}^{2}\right)^{-s}
$$


where $\lambda_{\psi}(r)$ is the $r$-th Hecke eigenvalue of $\psi$. We have

$$
\Lambda_{\psi \otimes \mathrm{sym}^{2}(\phi)}(1 / 2)=\frac{2}{2 \pi i} \int_{(2)} \Lambda_{\psi \otimes \mathrm{sym}^{2}(\phi)}(s+1 / 2) \frac{d s}{s} .
$$

So,

$$
L\left(1 / 2, \psi \otimes \operatorname{sym}^{2}\left(\phi_{j}\right)\right)=2 \sum_{m_{1}, m_{2} \geq 1} \lambda_{\psi}\left(m_{1}\right) a_{\Phi_{j}}\left(m_{1}, m_{2}\right)\left(m_{1} m_{2}^{2}\right)^{-1 / 2} V\left(\frac{m_{1} m_{2}^{2}}{t_{j}^{2}}\right)
$$

where

$$
V(y)=\frac{1}{2 \pi i} \int_{(2)} y^{-s} \frac{\gamma\left(1 / 2+s, \psi \otimes \operatorname{sym}^{2}\left(\phi_{j}\right)\right)}{\gamma\left(1 / 2, \psi \otimes \operatorname{sym}^{2}\left(\phi_{j}\right)\right)} \frac{d s}{s},
$$

and

$$
\begin{aligned}
\gamma\left(s, \psi \otimes \operatorname{sym}^{2}\left(\phi_{j}\right)\right)= & \pi^{-3 s} \Gamma\left(\frac{s+i t_{\psi}}{2}+i t_{j}\right) \Gamma\left(\frac{s+i t_{\psi}}{2}-i t_{j}\right) \Gamma\left(\frac{s+i t_{\psi}}{2}\right) \\
& \Gamma\left(\frac{s-i t_{\psi}}{2}+i t_{j}\right) \Gamma\left(\frac{s-i t_{\psi}}{2}-i t_{j}\right) \Gamma\left(\frac{s-i t_{\psi}}{2}\right) .
\end{aligned}
$$

Thus, we have

$$
\begin{aligned}
& \sum_{j \geq 1}\left(e^{-\left(\frac{t_{j}-T}{T^{1-\epsilon}}\right)^{2}}+e^{-\left(\frac{t_{j}+T}{T^{1-\epsilon}}\right)^{2}}\right) L\left(1, \mathrm{sym}^{2} \phi_{j}\right)\left|\left\langle\mu_{\phi_{j}}, \psi\right\rangle\right|^{2} \\
= & L\left(\frac{1}{2}, \psi\right)\left|\Gamma\left(\frac{1}{4}+\frac{1}{2} i t\right)\right|^{4} \sum_{t_{j} \geq 1} \frac{e^{-\left(\frac{t_{j}-T}{T^{1-\epsilon}}\right)^{2}}+e^{-\left(\frac{t_{j}+T}{T^{1-\epsilon}}\right)^{2}}}{t_{j}}\left|a_{j}(1)\right|^{2} L\left(1 / 2, \psi \otimes \operatorname{sym}^{2}\left(\phi_{j}\right)\right) \\
= & L\left(\frac{1}{2}, \psi\right)\left|\Gamma\left(\frac{1}{4}+\frac{1}{2} i t\right)\right|^{4} \sum_{t_{j} \geq 1} \frac{e^{-\left(\frac{t_{j}-T}{T^{1-\epsilon}}\right)^{2}}+e^{-\left(\frac{t_{j}+T}{T^{1-\epsilon}}\right)^{2}}}{t_{j}}\left|a_{j}(1)\right|^{2} \\
& \sum_{1} \lambda_{\psi}\left(m_{1}\right) a_{\Phi_{j}}\left(m_{1}, m_{2}\right)\left(m_{1} m_{2}^{2}\right)^{-1 / 2} V\left(\frac{m_{1} m_{2}^{2}}{t_{j}^{2}}\right) \\
= & L\left(\frac{1}{2}, \psi\right)\left|\Gamma\left(\frac{1}{4}+\frac{1}{2} i t\right)\right|^{4} \sum_{t_{j} \geq 1} \sum_{d \geq 1} \frac{\mu(d)}{d^{\frac{3}{2}}} \sum_{n_{1}, n_{2} \geq 1} \lambda_{\psi}\left(d n_{1}\right) V\left(\frac{d^{3} n_{1} n_{2}^{2}}{t_{j}^{2}}\right)\left(n_{1} n_{2}^{2}\right)^{-1 / 2} \\
& \frac{e^{-\left(\frac{t_{j}-T}{T^{1-\epsilon}}\right)^{2}}+e^{-\left(\frac{t_{j}+T}{T^{1-\epsilon}}\right)^{2}}}{t_{j}}\left|a_{j}(1)\right|^{2} \lambda_{\Phi_{j}}\left(n_{1}, 1\right) \lambda_{\Phi_{j}}\left(n_{2}, 1\right) \\
= & L\left(\frac{1}{2}, \psi\right)\left|\Gamma\left(\frac{1}{4}+\frac{1}{2} i t\right)\right|^{4} \sum_{t_{j} \geq 1} \sum_{d \geq 1} \frac{\mu(d)}{d^{\frac{3}{2}}} \sum_{s_{1}, s_{2}, n_{1}, n_{2} \geq 1} \lambda_{\psi}\left(d s_{1}^{2} t_{1}\right) V\left(\frac{d^{3} s_{1}^{2} t_{1} s_{2}^{4} t_{2}^{2}}{t_{j}^{2}}\right)\left(s_{1}^{2} t_{1} s_{2}^{4} t_{2}^{2}\right)^{-1 / 2} \\
& \frac{e^{-\left(\frac{t_{j}-T}{T^{1-\epsilon}}\right)^{2}}+e^{-\left(\frac{t_{j}+T}{T^{1-\epsilon}}\right)^{2}}\left|a_{j}(1)\right|^{2} \lambda_{j}\left(t_{1}^{2}\right) \lambda_{j}\left(t_{2}^{2}\right)}{t_{j}}
\end{aligned}
$$




$$
\begin{aligned}
= & L\left(\frac{1}{2}, \psi\right)\left|\Gamma\left(\frac{1}{4}+\frac{1}{2} i t\right)\right|^{4} \sum_{d \geq 1} \frac{\mu(d)}{d^{\frac{3}{2}}} \sum_{s_{1}, s_{2}, n_{1}, n_{2} \geq 1} \lambda_{\psi}\left(d s_{1}^{2} t_{1}\right)\left(s_{1}^{2} t_{1} s_{2}^{4} t_{2}^{2}\right)^{-1 / 2} \\
& \sum_{t_{j} \geq 1} V\left(\frac{d^{3} s_{1}^{2} t_{1} s_{2}^{4} t_{2}^{2}}{t_{j}^{2}}\right) \frac{e^{-\left(\frac{t_{j}-T}{T^{1-\epsilon}}\right)^{2}}+e^{-\left(\frac{t_{j}+T}{T^{1-\epsilon}}\right)^{2}}}{t_{j}}\left|a_{j}(1)\right|^{2} \lambda_{j}\left(t_{1}^{2}\right) \lambda_{j}\left(t_{2}^{2}\right)
\end{aligned}
$$

For the inner sum, by the Kuznetsov formula [25], we have

$$
\begin{aligned}
& \sum_{t_{j} \geq 1} V\left(\frac{d^{3} s_{1}^{2} t_{1} s_{2}^{4} t_{2}^{2}}{t_{j}^{2}}\right) \frac{e^{-\left(\frac{t_{j}-T}{T^{1-\epsilon}}\right)^{2}}+e^{-\left(\frac{t_{j}+T}{T^{1-\epsilon}}\right)^{2}}}{t_{j}}\left|a_{j}(1)\right|^{2} \lambda_{j}\left(t_{1}^{2}\right) \lambda_{j}\left(t_{2}^{2}\right) \\
&= \frac{\delta\left(t_{1}, t_{2}\right)}{\pi^{2}} \int_{-\infty}^{\infty} V\left(\frac{d^{3} s_{1}^{2} s_{2}^{4} t_{1} t_{2}^{2}}{t^{2}}\right)\left(e^{-\left(\frac{t-T}{T^{1-\epsilon}}\right)^{2}}+e^{-\left(\frac{t+T}{T^{1-\epsilon}}\right)^{2}}\right) \tanh (\pi t) d t \\
&- \frac{2}{\pi} \int_{0}^{\infty} V\left(\frac{d^{3} s_{1}^{2} t_{1} s_{2}^{4} t_{2}^{2}}{t^{2}}\right) \frac{e^{-\left(\frac{t-T}{T^{1-\epsilon}}\right)^{2}}+e^{-\left(\frac{t+T}{T^{1-\epsilon}}\right)^{2}}}{t|\zeta(1+2 i t)|^{2}} d_{i t}\left(t_{1}^{2}\right) d_{i t}\left(t_{2}^{2}\right) d t \\
&+ \sum_{c \geq 1} \frac{S\left(t_{1}^{2}, t_{2}^{2} ; c\right)}{c} \int_{-\infty}^{\infty} J_{2 i t}\left(\frac{4 \pi t_{1} t_{2}}{c}\right) V\left(\frac{d^{3} s_{1}^{2} s_{2}^{4} t_{1} t_{2}^{2}}{t^{2}}\right) \\
&\left(e^{-\left(\frac{t-T}{T^{1-\epsilon}}\right)^{2}}+e^{-\left(\frac{t+T}{T^{1-\epsilon}}\right)^{2}}\right) \frac{d t}{\cosh (\pi t)}
\end{aligned}
$$

Next, we will estimate the above three sums respectively.

\subsubsection{The Diagonal Term}

The diagonal term coming from (5.1) is

$$
\begin{array}{r}
L\left(\frac{1}{2}, \psi\right)\left|\Gamma\left(\frac{1}{4}+\frac{1}{2} i t_{\psi}\right)\right|^{4} \int_{-\infty}^{\infty}\left(e^{-\left(\frac{t-T}{T^{1-\epsilon}}\right)^{2}}+e^{-\left(\frac{t+T}{T^{1-\epsilon}}\right)^{2}}\right) \sum_{s_{2} \geq 1} s_{2}^{-2} \\
\sum_{s_{1} \geq 1} s_{1}^{-1} \lambda_{\psi}\left(s_{1}^{2}\right) V\left(\frac{s_{1}^{2} s_{2}^{4}}{T^{2}}\right) \tanh (\pi t) d t
\end{array}
$$

For the sum over $s_{1}$, we have

$$
\sum_{s_{1} \geq 1} s_{1}^{-1} \lambda_{\psi}\left(s_{1}^{2}\right) V\left(\frac{s_{1}^{2} s_{2}^{4}}{T^{2}}\right)=\frac{1}{2 \pi i} \int_{(2)} \sum_{s_{1} \geq 1} \frac{\lambda_{\psi}\left(s_{1}^{2}\right)}{s_{1}^{2 s+1}} U_{t}(s)\left(\frac{s_{2}^{4}}{T^{2}}\right)^{-s} \frac{d s}{s}
$$

where

$$
U_{t}(s)=\left(1+P_{t}(s)\right) \frac{\Gamma\left(\frac{s+1 / 2-i t_{\psi}}{2}\right) \Gamma\left(\frac{s+1 / 2+i t_{\psi}}{2}\right)}{\Gamma\left(\frac{1 / 2-i t_{\psi}}{2}\right) \Gamma\left(\frac{1 / 2+i t_{\psi}}{2}\right)},
$$


and

$$
P_{t}(s)=\sum_{1 \leq r \leq N} \frac{p_{r+1}(s)}{t^{r}}+O\left(\frac{|s|^{N+2}}{t^{N+1}}\right)
$$

is an analytic function in $\mathcal{R} s \geq-2 . p_{r+1}(s)$ is a polynomial of degree at most $r+1$. Also, we have [18]

$$
\sum_{s_{1} \geq 1} \frac{\lambda_{\psi}\left(s_{1}^{2}\right)}{s_{1}^{s}}=\frac{1}{\zeta(2 s)} L\left(s, \operatorname{sym}^{2} \psi\right) .
$$

Thus, moving the line of integration in the sum over $s_{1}$ to $\mathcal{R}(s)=-1 / 4+\epsilon$, we get

$$
\sum_{s_{1} \geq 1} s_{1}^{-1} \lambda_{\psi}\left(s_{1}^{2}\right) V\left(\frac{s_{1}^{2} s_{2}^{4}}{T^{2}}\right)=\frac{1}{\zeta(2)} L\left(1, \operatorname{sym}^{2} \psi\right)+O\left(T^{-1 / 2+\epsilon}\right)
$$

Therefore, we get the diagonal terms contribute

$$
\frac{\pi}{4} T^{1-\epsilon} L\left(1, \operatorname{sym}^{2} \psi\right) L\left(\frac{1}{2}, \psi\right)\left|\Gamma\left(\frac{1}{4}+\frac{1}{2} i t_{\psi}\right)\right|^{4}+O\left(T^{1 / 2+\epsilon}\right) .
$$

\subsubsection{The Non-diagonal Term}

Next, we estimate the non-diagonal term from (5.3)

$$
\begin{aligned}
& \sum_{d \geq 1} \frac{\mu(d)}{d^{\frac{3}{2}}} \sum_{s_{1}, s_{2}, t_{1}, t_{2} \geq 1} \lambda_{\psi}\left(d s_{1}^{2} t_{1}\right)\left(s_{1}^{2} t_{1} s_{2}^{4} t_{2}^{2}\right)^{-1 / 2} \sum_{c \geq 1} \frac{S\left(t_{1}^{2}, t_{2}^{2} ; c\right)}{c} \\
& \int_{-\infty}^{\infty} J_{2 i t}\left(\frac{4 \pi t_{1} t_{2}}{c}\right) V\left(\frac{d^{3} s_{1}^{2} t_{1} s_{2}^{4} t_{2}^{2}}{t^{2}}\right)\left(e^{-\left(\frac{t-T}{T^{1-\epsilon}}\right)^{2}}+e^{-\left(\frac{t+T}{T^{1-\epsilon}}\right)^{2}}\right) \frac{d t}{\cosh (\pi t)}
\end{aligned}
$$

Let $x=\frac{4 \pi t_{1} t_{2}}{c}$, the inner integral in the non-diagonal terms is

$$
\frac{1}{2} \int_{-\infty}^{\infty} \frac{J_{2 i t}(x)-J_{-2 i t}(x)}{\sinh \pi t} V\left(\frac{d^{3} s_{1}^{2} t_{1} s_{2}^{4} t_{2}^{2}}{t^{2}}\right)\left(e^{-\left(\frac{t-T}{T^{1-\epsilon}}\right)^{2}}+e^{-\left(\frac{t+T}{T^{1-\epsilon}}\right)^{2}}\right) \tanh (\pi t) d t .
$$

Since $\tanh (\pi t)=\operatorname{sgn}(t)+O\left(e^{-\pi|t|}\right)$ for large $|t|$, we can remove $\tanh (\pi t)$ by getting a negligible term $O\left(T^{-N}\right)$ for any $N>0$. Applying the Parseval identity, the Fourier transform in [27], (see also Bateman, volume 1, p. 59)

$$
\left(\frac{J_{2 i t}(x) \widehat{J-J i t}_{-2 i t}(x)}{\sinh (\pi t)}\right)(y)=-i \cos (x \cosh (\pi y)) .
$$

and the evaluation of the Fresnel integrals, the integral is 


$$
\begin{aligned}
& \frac{1}{2} \int_{-\infty}^{\infty} \frac{J_{2 i t}(x)-J_{-2 i t}(x)}{\sinh \pi t} V\left(\frac{d^{3} s_{1}^{2} t_{1} s_{2}^{4} t_{2}^{2}}{t^{2}}\right)\left(e^{-\left(\frac{t-T}{T^{1-\epsilon}}\right)^{2}}+e^{-\left(\frac{t+T}{T^{1-\epsilon}}\right)^{2}}\right) \tanh (\pi t) d t \\
& =\frac{1}{2} \int_{-\infty}^{\infty}\left(\frac{J_{2 i t}(x)-J_{-2 i t}(x)}{\sinh \pi t}\right)^{\wedge}(y)\left(V\left(\frac{d^{3} s_{1}^{2} t_{1} s_{2}^{4} t_{2}^{2}}{t^{2}}\right)\left(e^{-\left(\frac{t-T}{T^{1-\epsilon}}\right)^{2}}+e^{-\left(\frac{t+T}{T^{1-\epsilon}}\right)^{2}}\right)\right)^{\wedge}(y) d y \\
& +O\left(T^{-N}\right) \\
& =\frac{-i}{2} \int_{-\infty}^{\infty}(\cos (x \cosh (\pi y)))\left(V\left(\frac{d^{3} s_{1}^{2} t_{1} s_{2}^{4} t_{2}^{2}}{t^{2}}\right)\left(e^{-\left(\frac{t-T}{T^{1-\epsilon}}\right)^{2}}+e^{-\left(\frac{t+T}{T^{1-\epsilon}}\right)^{2}}\right)\right)^{\wedge}(y) d y+O\left(T^{-N}\right) \\
& =\frac{-i}{2} \int_{-\infty}^{\infty}\left(\cos \left(x+\frac{1}{2} \pi^{2} x y^{2}\right)\right)\left(V\left(\frac{d^{3} s_{1}^{2} t_{1} s_{2}^{4} t_{2}^{2}}{t^{2}}\right)\left(e^{-\left(\frac{t-T}{T^{1-\epsilon}}\right)^{2}}+e^{-\left(\frac{t+T}{T^{1-\epsilon}}\right)^{2}}\right)\right)^{\wedge}(y) d y+O\left(T^{-N}\right) \\
& =\frac{-i}{2} \int_{0}^{\infty}\left(\cos \left(x-y+\frac{\pi}{4}\right)\right)\left(V\left(\frac{d^{3} s_{1}^{2} t_{1} s_{2}^{4} t_{2}^{2}}{t^{2}}\right)\left(e^{-\left(\frac{t-T}{T^{1-\epsilon}}\right)^{2}}+e^{-\left(\frac{t+T}{T^{1-\epsilon}}\right)^{2}}\right)\right)\left(\sqrt{\frac{x y}{2}}\right) \frac{d y}{\sqrt{\pi y}} \\
& +O\left(T^{-N}\right) \\
& =\frac{-i}{2} \int_{0}^{\infty}\left(\cos \left(x-y+\frac{\pi}{4}\right)\right)\left(V\left(\frac{4 d^{3} s_{1}^{2} t_{1} s_{2}^{4} t_{2}^{2}}{x y}\right)\left(e^{-\left(\frac{\sqrt{\frac{x y}{2}}-T}{T^{1-\epsilon}}\right)^{2}}+e^{-\left(\frac{\sqrt{\frac{x y}{2}}+T}{T^{1-\epsilon}}\right)^{2}}\right)\right) \frac{d y}{\sqrt{\pi y}} \\
& +O\left(T^{-N}\right) \\
& =\frac{-i}{2} \int_{0}^{\infty}\left(\cos \left(4 \pi t_{1} t_{2} c^{-1}-y+\frac{\pi}{4}\right)\right)\left(V ( \frac { 4 d ^ { 3 } s _ { 1 } ^ { 2 } t _ { 1 } s _ { 2 } ^ { 4 } t _ { 2 } ^ { 2 } } { 4 \pi t _ { 1 } t _ { 2 } c ^ { - 1 } y } ) \left(e^{-\left(\frac{\sqrt{\frac{4 \pi t_{1} t_{2} c^{-1} y}{2}}-T}{T^{1-\epsilon}}\right)^{2}}\right.\right. \\
& \left.\left.+e^{-\left(\frac{\sqrt{\frac{4 \pi t_{1} t_{2} c^{-1} y}{2}}+T}{T^{1-\epsilon}}\right)^{2}}\right)\right) \frac{d y}{\sqrt{\pi y}}+O\left(T^{-N}\right)
\end{aligned}
$$

Thus, by partial integration in terms of $\mathrm{y}$, the non-diagonal terms is concentrated on

$$
T^{2}-T^{2-\epsilon} \ll t_{1} t_{2} c^{-1} y \ll T^{2} .
$$

So, we can assume $d^{3} s_{1}^{2} t_{1} s_{2}^{4} t_{2}^{2} \ll T^{2+\epsilon}$ since $V(\xi)$ has exponential decay as $\xi \rightarrow \infty$. By partial integration, the terms with $c \gg T^{\epsilon}$ and $t_{1} t_{2} \ll T^{2-4 \epsilon}$ contribute $\mathrm{O}(1)$. So we can assume $c \ll T^{\epsilon}$ and $t_{1} t_{2} \gg T^{2-4 \epsilon}$, therefore we have $t_{2} \ll T^{5 \epsilon}$, also we have the sum over $s_{1}$ and $s_{2}$ converges. Let $t=\frac{\sqrt{2 \pi t_{1} t_{2} c^{-1} y}}{T}$, the inner integral is $\frac{T \sqrt{c}}{2 \pi t_{1} t_{2}} \int_{0}^{\infty}\left(e^{-\left((t-1) / T^{-\epsilon}\right)^{2}}-e^{-\left((t+1) / T^{-\epsilon}\right)^{2}}\right)\left(\cos \left(4 \pi t_{1} t_{2} c^{-1}-(t T)^{2} c /\left(2 \pi t_{1} t_{2}\right)+\frac{\pi}{4}\right)\right) V\left(\frac{4 d^{3} s_{1}^{2} t_{1} s_{2}^{4} t_{2}^{2}}{t^{2} T^{2}}\right) d t$ From the bound ([17] Theorem 8.1),

$$
\sum_{r \leq R} \lambda_{\psi}(r) r^{-1 / 2} \ll_{\epsilon} R^{\epsilon}
$$


and the Hecke relation

$$
\lambda_{\psi}\left(r_{1} r_{2}\right)=\sum_{d \mid\left(r_{1}, r_{2}\right)} \mu(d) \lambda_{\psi}\left(r_{1} / d\right)\left(r_{2} / d\right)
$$

and partial summation, we get the non-diagonal terms contribute $O\left(T^{5 \epsilon}\right)$.

\subsubsection{The Continuous Spectrum Part}

To evaluate the continuous part from (5.2), we need rewrite

$$
\begin{aligned}
& \sum_{d \geq 1} \frac{\mu(d)}{d^{\frac{3}{2}}} \sum_{s_{1}, s_{2}, t_{1}, t_{2} \geq 1} \lambda_{\psi}\left(d s_{1}^{2} t_{1}\right)\left(s_{1}^{2} t_{1} s_{2}^{4} t_{2}^{2}\right)^{-1 / 2} \\
& \int_{0}^{\infty} V\left(\frac{d^{3} s_{1}^{2} t_{1} s_{2}^{4} t_{2}^{2}}{t^{2}}\right) \frac{e^{-\left(\frac{t-T}{T^{1-\epsilon}}\right)^{2}}+e^{-\left(\frac{t+T}{T^{1-\epsilon}}\right)^{2}}}{t|\zeta(1+2 i t)|^{2}} d_{i t}\left(t_{1}^{2}\right) d_{i t}\left(t_{2}^{2}\right) d t
\end{aligned}
$$

with respect to $L$-function, then we can use some mean value bounds of $L$-function to show the continuous part is also small. By Theorem 3 in [21], we have

$$
\int_{0}^{T}\left|L\left(\frac{1}{2}+i t, \varphi_{j}\right)\right|^{2} d t=T P(\log T)+O\left(T^{\frac{2}{3}+\epsilon}\right),
$$

here $P(t)$ is a polynomial of degree 4 . So

$$
\int_{T}^{T+T^{\frac{2}{3}}}\left|L\left(\frac{1}{2}+i t, \varphi_{j}\right)\right|^{2} d t=O\left(T^{\frac{2}{3}+\epsilon}\right)
$$

The Eisenstein series $E(z, s)$ defined by

$$
E(z, s)=\frac{1}{2} \sum_{(c, d) \geq 1} \frac{y^{s}}{(c z+d)^{2 s}}
$$

has Fourier expansion

$$
E(z, s)=y^{s}+\phi(s) y^{1-s}+\sum_{n \neq 0} \phi(n, s) W_{s}(n z)
$$

where $W_{s}(z)$ is the Whittaker function given by

$$
W_{s}(z)=2|y|^{\frac{1}{2}} K_{s-\frac{1}{2}}(2 \pi|y|) e(x)
$$


and $K_{s}(y)$ is the $K$-Bessel function,

$$
\phi(s)=\sqrt{\pi} \frac{\Gamma\left(s-\frac{1}{2}\right) \zeta(2 s-1)}{\Gamma(s) \zeta(2 s)}
$$

with $\zeta(s)$ be the Riemann zeta function and

$$
\phi(n, s)=\pi^{s} \Gamma(s)^{-1} \zeta(2 s)^{-1}|n|^{-\frac{1}{2}} \eta(n, s)
$$

with

$$
\eta(n, s)=\sum_{a d=n}\left(\frac{a}{d}\right)^{s-\frac{1}{2}} .
$$

For $E\left(z, \frac{1}{2}+i t\right)$, we associate the $L$-function

$$
L(s, E)=\prod_{p}\left(1-p^{-s+i t}\right)^{-1}\left(1-p^{-s-i t}\right)^{-1},
$$

and denote

$$
L\left(s, \operatorname{sym}^{2} E\right)=\zeta(s) \prod_{p}\left(1-p^{-s+2 i t}\right)^{-1}\left(1-p^{-s-2 i t}\right)^{-1},
$$

If

$$
L(s, \psi)=\prod_{p}\left(1-\alpha_{p} p^{-s}\right)^{-1}\left(1-\beta_{p} p^{-s}\right)^{-1},
$$

we have

$$
L\left(s, \psi \otimes \operatorname{sym}^{2} E\right)=L(s-2 i t, \psi) L(s+2 i t, \psi) L(s, \psi)
$$

Thus

$$
L\left(\frac{1}{2}, \psi \otimes \operatorname{sym}^{2} E\right)=\left|L\left(\frac{1}{2}+2 i t, \psi\right)\right|^{2} L\left(\frac{1}{2}, \psi\right) .
$$

For Eisenstein series, we can take the advantage of unfolding integral,

$$
\begin{aligned}
I_{j}(s) & =\int_{X} \varphi_{j}(z) E\left(z, \frac{1}{2}+i t\right) E(z, s) \frac{d x d y}{y^{2}} \\
& =\int_{0}^{\infty} \int_{0}^{1} \varphi_{j}(z) E\left(z, \frac{1}{2}+i t\right) y^{s} \frac{d x d y}{y^{2}}
\end{aligned}
$$




$$
\begin{aligned}
= & \int_{0}^{\infty} \int_{0}^{1}\left(y^{\frac{1}{2}} \sum_{n=1}^{\infty} \lambda_{j}(n) K_{i t_{j}}(2 \pi n y) \cos (2 \pi n x)\right)\left(y^{\frac{1}{2}+i t}+\varphi\left(\frac{1}{2}+i t\right) y^{\frac{1}{2}-i t}+\right. \\
& \left.\frac{2 y^{\frac{1}{2}}}{\xi(1+2 i t)} \sum_{n=1}^{\infty} n^{i t} \sigma_{-2 i t}(n) K_{i t}(2 \pi n y) \cos (2 \pi n x) y^{s}\right) \frac{d x d y}{y^{2}} \\
= & \frac{1}{\xi(1+2 i t)} \int_{0}^{\infty}\left(\sum_{n=1}^{\infty} \lambda_{j}(n) K_{i t_{j}}(2 \pi n y) n^{i t} \sigma_{-2 i t}(n) K_{i t}(2 \pi n y)\right) y^{s} \frac{d x d y}{y} \\
= & \frac{1}{\xi(1+2 i t)}\left(\sum_{n=1}^{\infty} \frac{\lambda_{j}(n) n^{i t} \sigma_{-2 i t}(n)}{n^{s}}\right) \int_{0}^{\infty} K_{i t_{j}}(2 \pi y) K_{i t}(2 \pi y) y^{s} \frac{d x d y}{y} \\
= & \frac{L\left(\varphi_{j}, s-i t\right) L\left(\varphi_{j}, s+i t\right)}{\zeta(2 s)} \frac{2 \pi^{-s}}{\xi(1+2 i t)} \frac{\Gamma\left(\frac{s+i t_{j}+i t}{2}\right) \Gamma\left(\frac{s+i t_{j}-i t}{2}\right) \Gamma\left(\frac{s-i t_{j}+i t}{2}\right) \Gamma\left(\frac{s-i t_{j}-i t}{2}\right)}{\Gamma(s)}
\end{aligned}
$$

Thus,

$$
\left\langle\varphi_{j}, \mu_{t}\right\rangle=2 \pi^{-2 i t} \frac{\left|\Gamma\left(\frac{1}{4}+\frac{i t_{j}}{2}\right)\right|^{2}}{|\zeta(1+2 i t)|^{2}} \frac{\Gamma\left(\frac{1}{4}-\frac{i t_{j}}{2}-i t\right) \Gamma\left(\frac{1}{4}+\frac{i t_{j}}{2}-i t\right)}{\left|\Gamma\left(\frac{1}{2}+i t\right)\right|^{2}} L\left(\varphi_{j}, \frac{1}{2}\right) L\left(\varphi_{j}, \frac{1}{2}-2 i t\right)
$$

We have

$$
L\left(\frac{1}{2}, \psi \otimes \operatorname{sym}^{2} E\right)=\left|L\left(\frac{1}{2}+2 i t, \psi\right)\right|^{2} L\left(\frac{1}{2}, \psi\right) .
$$

On the other hand

$$
L\left(\frac{1}{2}, \psi \otimes \operatorname{sym}^{2} E\right)=2 \sum_{m_{1}, m_{2} \geq 1} \lambda_{\psi}\left(m_{1}\right) a_{E}\left(m_{1}, m_{2}\right)\left(m_{1} m_{2}^{2}\right)^{-1 / 2} V\left(\frac{m_{1} m_{2}^{2}}{t^{2}}\right)
$$

where

$$
V(y)=\frac{1}{2 \pi i} \int_{(2)} y^{-s} \frac{\gamma\left(1 / 2+s, \psi \otimes \operatorname{sym}^{2}(E)\right)}{\gamma\left(1 / 2, \psi \otimes \operatorname{sym}^{2}(E)\right)} \frac{d s}{s}
$$

Thus, we have

$$
\begin{aligned}
& \int_{0}^{\infty}\left(e^{-\left(\frac{t-T}{T^{1-\epsilon}}\right)^{2}}+e^{-\left(\frac{t+T}{T^{1-\epsilon}}\right)^{2}}\right) L\left(1, \operatorname{sym}^{2} E\left(\frac{1}{2}+i t\right)\right)|\langle\mu t, \psi\rangle|^{2} d t \\
= & L\left(\frac{1}{2}, \psi\right)\left|\Gamma\left(\frac{1}{4}+\frac{1}{2} i t_{\psi}\right)\right|^{4} \int_{0}^{\infty} \frac{e^{-\left(\frac{t-T}{T^{1-\epsilon}}\right)^{2}}+e^{-\left(\frac{t+T}{T^{1-\epsilon}}\right)^{2}}}{t}\left|a_{E}(1)\right|^{2} L\left(1 / 2, \psi \otimes \operatorname{sym}^{2} E\right) d t
\end{aligned}
$$




$$
\begin{aligned}
& =L\left(\frac{1}{2}, \psi\right)\left|\Gamma\left(\frac{1}{4}+\frac{1}{2} i t_{\psi}\right)\right|^{4} \int_{0}^{\infty} \frac{e^{-\left(\frac{t-T}{T^{1-\epsilon}}\right)^{2}}+e^{-\left(\frac{t+T}{T^{1-\epsilon}}\right)^{2}}}{t}\left|a_{E}(1)\right|^{2} \\
& \sum_{m_{1}, m_{2} \geq 1} \lambda_{\psi}\left(m_{1}\right) a_{E}\left(m_{1}, m_{2}\right)\left(m_{1} m_{2}^{2}\right)^{-1 / 2} V\left(\frac{m_{1} m_{2}^{2}}{t^{2}}\right) d t \\
& =L\left(\frac{1}{2}, \psi\right)\left|\Gamma\left(\frac{1}{4}+\frac{1}{2} i t_{\psi}\right)\right|^{4} \int_{0}^{\infty} \sum_{d \geq 1} \frac{\mu(d)}{d^{\frac{3}{2}}} \sum_{n_{1}, n_{2} \geq 1} \lambda_{\psi}\left(d n_{1}\right) V\left(\frac{d^{3} n_{1} n_{2}^{2}}{t^{2}}\right)\left(n_{1} n_{2}^{2}\right)^{-1 / 2} \\
& \frac{e^{-\left(\frac{t-T}{T^{1-\epsilon}}\right)^{2}}+e^{-\left(\frac{t+T}{T^{1-\epsilon}}\right)^{2}}}{t}\left|a_{E}(1)\right|^{2} \lambda_{E}\left(n_{1}, 1\right) \lambda_{E}\left(n_{2}, 1\right) d t \\
& =L\left(\frac{1}{2}, \psi\right)\left|\Gamma\left(\frac{1}{4}+\frac{1}{2} i t_{\psi}\right)\right|^{4} \int_{0}^{\infty} \sum_{d \geq 1} \frac{\mu(d)}{d^{\frac{3}{2}}} \sum_{s_{1}, s_{2}, n_{1}, n_{2} \geq 1} \lambda_{\psi}\left(d s_{1}^{2} t_{1}\right) V\left(\frac{d^{3} s_{1}^{2} t_{1} s_{2}^{4} t_{2}^{2}}{t^{2}}\right) \\
& \left(s_{1}^{2} t_{1} s_{2}^{4} t_{2}^{2}\right)^{-1 / 2} \frac{e^{-\left(\frac{t-T}{T^{1-\epsilon}}\right)^{2}}+e^{-\left(\frac{t+T}{T^{1-\epsilon}}\right)^{2}}}{t}\left|a_{E}(1)\right|^{2} \lambda\left(t_{1}^{2}\right) \lambda\left(t_{2}^{2}\right) d t \\
& =L\left(\frac{1}{2}, \psi\right)\left|\Gamma\left(\frac{1}{4}+\frac{1}{2} i t_{\psi}\right)\right|^{4} \sum_{d \geq 1} \frac{\mu(d)}{d^{\frac{3}{2}}} \sum_{s_{1}, s_{2}, n_{1}, n_{2} \geq 1} \lambda_{\psi}\left(d s_{1}^{2} t_{1}\right)\left(s_{1}^{2} t_{1} s_{2}^{4} t_{2}^{2}\right)^{-1 / 2} \\
& \int_{0}^{\infty} V\left(\frac{d^{3} s_{1}^{2} t_{1} s_{2}^{4} t_{2}^{2}}{t^{2}}\right) \frac{e^{-\left(\frac{t-T}{T^{1-\epsilon}}\right)^{2}}+e^{-\left(\frac{t+T}{T^{1-\epsilon}}\right)^{2}}}{t}\left|a_{E}(1)\right|^{2} \lambda\left(t_{1}^{2}\right) \lambda\left(t_{2}^{2}\right) d t
\end{aligned}
$$

Therefore, the continuous part contributes

$$
\begin{aligned}
& \int_{0}^{\infty}\left(e^{-\left(\frac{t-T}{T^{1-\epsilon}}\right)^{2}}+e^{-\left(\frac{t+T}{T^{1-\epsilon}}\right)^{2}}\right) \frac{1}{|\zeta(1+2 i t)|^{2}}\left|L\left(\frac{1}{2}+i t, \psi\right)\right|^{2} \\
& \frac{\left|\Gamma\left(\frac{1}{4}-\frac{i t_{\psi}}{2}-i t\right) \Gamma\left(\frac{1}{4}+\frac{i t_{\psi}}{2}-i t\right)\right|^{2}}{\left|\Gamma\left(\frac{1}{2}+i t\right)\right|^{4}} d t
\end{aligned}
$$

By Stirling formula and the Jutila's bound the subconvexity bound

$$
L\left(\frac{1}{2}+i t, \psi_{j}\right) \ll\left(t_{j}^{2}+t\right)^{1 / 3+\epsilon},
$$

we obtain the continuous part contributes $O\left(T^{\frac{1}{2}+\epsilon}\right)$.

\subsection{Conclusion}

Combining last section's results, we conclude that

$$
\begin{aligned}
& \sum_{j \geq 1}\left(e^{-\left(\frac{t_{j}-T}{T^{1-\epsilon}}\right)^{2}}+e^{-\left(\frac{t_{j}+T}{T^{1-\epsilon}}\right)^{2}}\right) L\left(1, \operatorname{sym}^{2} \phi_{j}\right)\left|\left\langle\mu_{\phi_{j}}, \psi\right\rangle\right|^{2} \\
= & \frac{\pi}{4} T^{1-\epsilon} L\left(1, \operatorname{sym}^{2} \psi\right) L\left(\frac{1}{2}, \psi\right)\left|\Gamma\left(\frac{1}{4}+\frac{1}{2} i t_{\psi}\right)\right|^{4}+O\left(T^{1 / 2+\epsilon}\right) .
\end{aligned}
$$


We have

$$
L\left(1, \operatorname{sym}^{2} \psi\right)=2<\psi, \psi>\cosh \left(\pi t_{\psi}\right)
$$

since $\psi(z)$ is normalized so that its first Fourier coefficient is 1 [31]. Thus, we obtain the eigenvalue of $B$ at $\psi$ is

$$
L\left(\frac{1}{2}, \psi\right) \frac{\left|\Gamma\left(\frac{1}{4}-\frac{i t_{\psi}}{2}\right)\right|^{4}}{2 \pi\left|\Gamma\left(\frac{1}{2}-i t_{\psi}\right)\right|^{2}} .
$$

Next, let us recall the classical variance of the geodesic flow which is studied by Ratner.

We realize the upper half plane $\mathbb{H}$ as the homogeneous space $S L_{2}(\mathbb{R}) / S O_{2}(\mathbb{R})$ and $Y=S L_{2}(\mathbb{Z}) \backslash S L_{2}(\mathbb{R})$ as the unit cotangent bundle for $X$. The motion along geodesics on $X$ gives rise to a Hamiltonian flow $\mathcal{G}_{t}$ on $Y$

$$
\Gamma g \rightarrow \Gamma g\left(\begin{array}{cc}
e^{\frac{t}{2}} & 0 \\
0 & e^{-\frac{t}{2}}
\end{array}\right), \quad t \in \mathbf{R}
$$

Since the geodesic flow on a negative curvature manifold is ergodic [15], we can consider this Hamiltonian flow as a classically chaotic system. Ratner [39] has shown that, for a classical observable $\psi \in C_{0,0}^{\infty}(Y)$, the fluctuations along a generic orbit of the geodesic flow obey the central limit theorem, i.e. the distribution of

$$
\frac{1}{\sqrt{T}} \int_{0}^{T} \psi\left(\mathcal{G}_{t}(g)\right) d t
$$

become Gaussian with mean 0 and the variance given by the Hermitian form on $C_{0,0}^{\infty}(Y)$

$$
V_{\mathrm{C}}(\phi, \psi)=\int_{-\infty}^{\infty} \int_{\Gamma \backslash S L(2, \mathbf{R})} \phi\left(g\left(\begin{array}{cc}
e^{\frac{t}{2}} & 0 \\
0 & e^{-\frac{t}{2}}
\end{array}\right)\right) \overline{\psi(g)} d g d t,
$$

and $V_{C}$ is diagonalized by the Maass forms on $S L_{2}(\mathbb{R}) / S O_{2}(\mathbb{R})$. Let $\psi$ be a Maass form on $S L_{2}(\mathbb{R}) / S O_{2}(\mathbb{R})$ with Laplacian engenvalue $\frac{1}{4}+r^{2}$, we have explicitly [46]

$$
\int_{\Gamma \backslash S L(2, \mathbf{R})} \psi\left(g\left(\begin{array}{cc}
e^{\frac{t}{2}} & 0 \\
0 & e^{-\frac{t}{2}}
\end{array}\right)\right) \overline{\psi(g)} d g=P_{-\frac{1}{2}+i r}(\cosh t),
$$


where $P_{-\frac{1}{2}+i r}(z)$ is the generalized Legendre function and we have the following integral representation [17]

$$
P_{-\frac{1}{2}+i r}(z)=\frac{1}{\pi} \int_{0}^{\pi}\left(z+\sqrt{z^{2}-1} \cos x\right)^{-\frac{1}{2}+i r} d x
$$

Thus,

$$
V_{C}(\psi, \psi)=\int_{\infty}^{\infty} P_{-\frac{1}{2}+i r}(\cosh t) d t
$$

which can be explicitly calculated using the integral table [11] and we obtain

$$
V_{\mathrm{C}}(\psi, \psi)=\frac{\left|\Gamma\left(\frac{1}{4}-\frac{i r}{2}\right)\right|^{4}}{2 \pi\left|\Gamma\left(\frac{1}{2}-i r\right)\right|^{2}}
$$

This completes the proof of Theorem 1.4.

Remark 5.1. As a by-product, we obtain that, for Maass-Hecke form $\psi, L(1 / 2, \psi \otimes$ $\left.\operatorname{sym}^{2}\left(\phi_{j}\right)\right)$ is non-vanishing for some $\phi_{j}$ and the central value $L\left(\frac{1}{2}, \psi\right)$ is non-negative. 


\section{BIBLIOGRAPHY}

[1] E. Artin, Ein mechanisches System mit quasi-ergodischen Bahnen, Abh. Math. Sem. d. Hamburgischen Universitat, 3 (1924) 170-175.

[2] D. Bump, Automorphic Forms on $G L(3, R)$, Lecture Notes in Mathematics, Vol. 1083.

[3] D. Bump, The Rankin-Selberg method: a survey, Number Theory, Trace Formulas and Discrete Groups, Academic Press, 1989, 49-109.

[4] Y. Colin de Verdiere, Ergodicite et fonctions propres du laplacien. Com. Math. Phy. $102(1985)$

[5] W. Duke, J. Friedlander and H. Iwaniec, Bound for automorphic L-functions. Invent. Math. 112 (1993), 1-8.

[6] J.-M. Deshouillers and H. Iwaniec, Kloosterman Sums and Fourier Coefficients of Cusp Forms, Invent. Math. 70, 219-288, 1982.

[7] T. Dunster, Bessel functions of purely imaginary order, with an application to secondorder linear differential equations having a large parameter. SIAM J. Math. Anal. Vol.21 (1990), No.4, 995-1018.

[8] A. Erdelyi et al., Higher transcendental functions Vol. 2 (1953), McGraw-Hill Book Company.

[9] B. Eckhardt, Fishman et al. Approach to ergodicity in quantum wave functions, Phys. Rev. E 52 (1995)

[10] M. Feingold, A. Peres, Distributin of matrix elements of chaotic systems, Phy. Rev. A 34 (1986).

[11] I. S. Gradshteyn and I. M. Ryzhik, Table of integrals, series, and products, 4th ed., Academic Press, New York, 1965 prepared by Ju. V. Geronimus and M. Ju. Certlin. Translated from the Russian by Scripta Technica, Inc. Translation edited by Alan Jeffrey.

[12] J. Hoffstein and P. Lockhart, Coefficients of Maass forms and the Siegel zero, Ann. of Math.(2) 140 (1994), no. 1, 161-181, Appendix: An effective zero-free region, by Dorian Goldfeld, Hoffstein and Daniel Lieman.

[13] R. Holowinsky and K. Soundararajan, Mass equidistribution of Hecke eigenforms. arXiv preprint. 
[14] Hejhal and Rackner, On the topography of Maass wave forms, Exper. Math. 1992, 275-305.

[15] E. Hopf,Statistik der geodeischen Linien in Mannigfaltigkeiten negativer Krmmung", Leipzig Ber. Verhandl. Shs. Akad. Wiss.(1939) 91: 261-304 .

[16] H. Iwaniec, Topics in classical automorphic forms, Graduate Studies in Mathematics, vol. 17, American Mathematical Society, Rhode Island, 1997.

[17] H. Iwaniec, Spectral theory of automorphic forms, Graduate Studies in Mathematics, 53. American Mathematical Society, Providence, RI; Revista Matemtica Iberoamericana, Madrid, 2002.

[18] H. Iwaniec, E. Kowalski, Analytic number theory. American Mathematical Society Colloquium Publications, 53. American Mathematical Society, Providence, RI, 2004.

[19] Iwaniec, H.; Luo, W.; Sarnak, P.Low lying zeros of families of L-functions. Inst. Hautes eudes Sci. Publ. Math. No. 91 (2000), 55-131 (2001)

[20] D. Jakobson, Equidistribution of cusp forms on $P S L_{2}(Z) \backslash P S L_{2}(R)$, Ann. Inst. Fourier, 1997.

[21] M. Jutila, Mean values of Dirichlet series via Laplace transform, Analytic number theory (Kyoto, 1996), 169-207, London Math. Soc. Lecture Note Ser., 247, Cambridge Univ. Press, Cambridge, 1997.

[22] M. Jutila, On exponential sums involving the divisor function, Journal fur die reine und angewandte Mathematik 355 (1985), 173-190

[23] Jutila, M. The spectral mean square of Hecke L-functions on the critical line. Publ. Inst. Math. (Beograd) (N.S.) 76(90) (2004), 41-55.

[24] P. Kurlberg and Z. Rudnick, On the distribution of matrix elements for the quantum cat map, Ann. of Math. (2) 161 (2005), no. 1,489-507.

[25] N. V. Kuznetsov, Petersson's Conjecture for Cusp Forms of Weight Zero and Linnik's Conjecture. Math. USSR Sbornik 29 1981, 299-342.

[26] E. Lindenstrauss, Invariant measures and arithmetic quantum unique ergodicity. Ann. of Math. (2) 163 (2006), no. 1, 165-219.

[27] J. Liu and Y. Ye, Subconvexity for Rankin-Selberg L-Functions of Maass Forms, GAFA. 2002, Vol. 12, 1296-1323.

[28] W. Luo, Z. Rudnick and P. Sarnak, The variance of arithmetic measures associated to closed geodesics on the modular surface, ArXiv preprint.

[29] W. Luo, Zeros of Hecke L-functions associated with cusp forms. Acta Arith. 71 (1995), no. 2, 139-158. 
[30] W. Luo, Spectral mean-value of automorphic L-functions at special points. Analytic number theory, Vol. 2 (Allerton Park, IL, 1995), 621-632. Progr. Math., 139, (1996).

[31] W. Luo, Values of symmetric square L-functions at 1, J. Reine Angew. Math. 506 (1999) 215-235.

[32] W. Luo and P. Sarnak, Quantum ergodicity of eigenfunctions on $P S L_{2}(Z) \backslash H$, Inst. Hautes tudes Sci. Publ. Math. No. 81 (1995), 207-237.

[33] W. Luo and P. Sarnak, Maass equidistribution for Hecke eigenforms, Comm. Pure Appl. Math. 56 (2003), no. 7, 874-891..

[34] W. Luo and P. Sarnak, Quatum variance for Hecke eigenforms, 2004 Ann. Sci. cole Norm. Sup. (4) 37 (2004), no. 5, 769-799.

[35] T. Meurman, On exponential sums involving the Fourier coefficients of Maass wave forms. Journal fur die reine und angewandte Mathematik 384 (1988), 192-207

[36] F. Olver, J. Asymptotics and special functions. Computer Science and Applied Mathematics. Academic Press [A subsidiary of Harcourt Brace Jovanovich, Publishers], (1974).

[37] S. Ramanujan, Some formulae in the arithmetic theory of numbers, Messenger of Math., 45 (1916), 81-84.

[38] M. Ratner, The rate of mixing for geodesic and horocycle flows, Ergodic Theory Dynam. Sys. 7 (1987) 267-288.

[39] M. Ratner, The central limit theorem for geodesic flows on $n$-dimensional manifolds of negative curvature Israel J. Math. 16 (1973) 181-197.

[40] Z. Rudnick and P. Sarnak, The behavior of eigenstates of hyperbolic manifolds, Com. Math. Phys. 1994

[41] P. Sarnak, Arithmetic Quantum Chaos, The Schur Lectures, 2003.

[42] P. Sarnak, Spectra of Hyperbolic Surfaces, Notes for Baltimore Lectures, 2003.

[43] P. Sarnak, Estimates for Rankin-Selberg L-functions and quantum unique ergodicity. J. Funct. Anal. 184 (2001), no. 2, 419-453.

[44] A. Schnirelman, Ergodic properties of eigenfunctions. Usp. Math. Nauk. 29 (1974), 181-182.

[45] K. Soundararajan Quantum unique ergodicity for $S L_{2}(Z) \backslash \mathbb{H}$, Arxiv preprint.

[46] A. Terras, Harmonic Analysis on Symmetric Spaces and Applications I. SpringerVerlag, New York, 1973

[47] E. C. Titchmarsh, The theory of the Riemann zeta-function, 2nd ed., Edited and with a preface by D. R. Heath-Brown. The Clarendon Press, Oxford University Press, New York, 1986. 
[48] G. N. Watson, A Treatise on the Theory of Bessel Functions, Cambridge University Press, Cambridge, England; The Macmillan Company, New York, 1944.

[49] T. Watson, Central Value of Rankin Triple L-function for Unramified Maass Cusp Forms, Princeton thesis, 2004.

[50] H. Weyl, Zur Abschatzung von $\xi(1+i t)$. Math. Z. 10 (1921), p. 88-101.

[51] S. Zelditch, On the rate of quantum ergodicity, Comm. Math. Phys. 160 (1994)81-92.

[52] S. Zelditch, Uniform distribution of eigenfunctions on compact hyperbolic surfaces, Duke Math. J. 55(1987). 